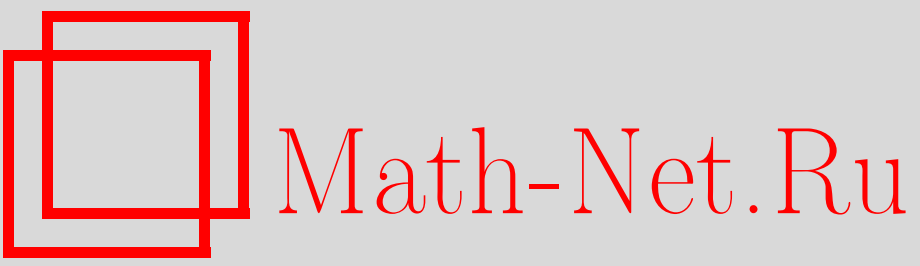

С. М. Натанзон, Модули римановых поверхностей, пространства типа Гурвица и их супераналоги, УМH, 1999, том 54, выпуск 1, 61-116

DOI: https://doi.org/10.4213/rm117

Использование Общероссийского математического портала Math-Net.Ru подразумевает, что вы прочитали и согласны с пользовательским соглашением

http://www . mathnet.ru/rus/agreement

Параметры загрузки:

IP : 18.234 .197 .8

26 апреля 2023 г., 15:40:51 


\title{
МОДУЛИ РИМАНОВЫХ ПОВЕРХНОСТЕЙ, ПРОСТРАНСТВА ТИПА ГУРВИЦА И ИХ СУПЕРАНАЛОГИ
}

\author{
C. M. НАТАНЗОН
}

СОДЕРЖАНИЕ

Введение

$\S 1$. Фуксовы группы и их последовательные образующие . . . . . . . . . . . . . 63

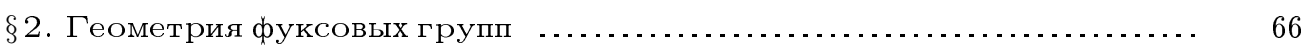

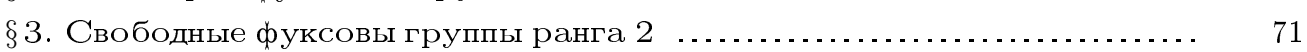

$\S 4$. Пространства типа Фрике-Клейна-Тайхмюллера ............. 77

$\S 5$. Модули римановых поверхностей ........................... 81

$\S 6$. Пространство голоморффных морфизмов римановых поверхностей ... 83

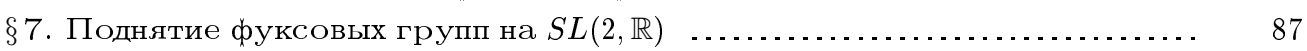

$\S 8$. Топологическая классификация функций Арфа и пар функций Арфа 92

$\S 9$. Топологическая классификация независимых систем функций Арфа

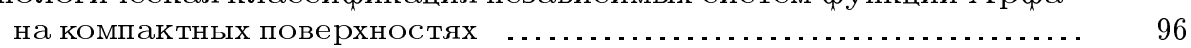

$\S 10$. Пространство модулей спинорных расслоений ранга 1 . . . . . . . . 98

$\S 11$. Суперфуксовы группы, суперримановы поверхности и их тополо-

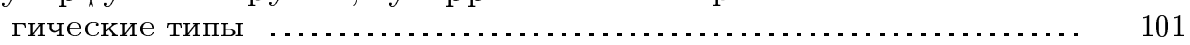

$\S 12$. Модули суперримановых поверхностей . . . . . . . . . . . . . . . . . . . . . . 103

$\S 13 . N=2$ суперфуксовы группы. $N=2$ суперримановы поверхности и их топологические инварианты .................................. 107

$\S 14$. Модули $N=2$ суперримановых поверхностей ... . . . . . . . . . . . . 109

$\S 15$. Суперголоморффные морффизмы римановых суперповерхностей $\ldots .$.

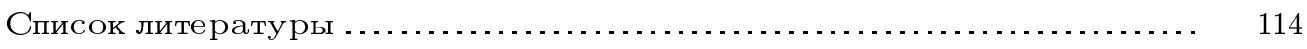

\section{Введение}

На протяжении всего 20 века пространства модулей римановых поверхностей являлись объектом постоянного интереса математиков. В последние два десятилетия эти исследования получили новый стимул в связи с открытием глубоких связей пространств модулей римановых поверхностей с теорией струн - современного варианта

Работа выполнена при частичной финансовой поддержке грантов: РФФИ-98-01-00612, INTAS-96-0713 и DGICYT. 
единой теории поля [1]. Теория струн приводит к рассмотрению некоммутативного аналога римановых поверхностей: римановых $N=1$ суперповерхностей [2], [3]. Математическая часть теории при этом сводится к изучению супераналога меры Мамфорда на пространстве модулей римановых $N=1$ суперповерхностей [4], [5]. Дальнейшее развитие теории приводит к появлению римановых $N=2$ суперповерхностей [6]-[8].

До недавнего времени значительно меньше внимания уделялось изучению пространств голоморфных отображений римановых поверхностей. В случае тождественных отображений это пространство совпадает с пространством модулей римановых поверхностей. В случае отображений на сферу оно совпадает с пространством мероморфных функций, рассматривавшимся в работах Гурвица [9]. В последние годы выяснилось, что пространства голоморфных отображений играют центральную роль в двумерной топологической теории поля: к ним, по-видимому, сводятся полупростые фробениусовы многообразия в смысле Дубровина [10], [11].

Естественной структурой фробениусова многообразия обладают, в частности, пространства квантовых когомологий четной размерности [12], [13]. Для изучения квантовых когомологий нечетной размерности необходим супераналог фробениусова многообразия [12], [14]. Это делает актуальным исследование пространств суперголоморфных отображений римановых суперповерхностей. Другим источником возникновения суперголоморфных отображений являются суперконформные инстантоны [15]-[17].

Обзор посвящен описанию топологической структуры пространств модулей римановых поверхностей, пространств модулей римановых суперповерхностей, пространств голоморфных отображений римановых поверхностей и пространств суперголоморфных отображений римановых суперповерхностей. Мы рассматриваем только случай гиперболических поверхностей. Для негиперболических поверхностей (сфер с менее чем тремя проколами и торов) теория значительно проще, но требует других конструкций.

Топологическое описание состоит из: 1) описания компонент связности этих пространств, 2) представления каждой из компонент в виде $T / \operatorname{Mod}$, где $T$ - пространство, гомеоморфное линейному пространству или его супераналогу, a Mod - дискретная группа.

В классическом случае римановых поверхностей описание такого типа было впервые предложено Фрике и Клейном [18]. В этом случае компонента связности определяется топологическим типом поверхности. Мы доказываем, что в случае суперповерхностей надо фиксировать еще и тип индуцированной функции Арфа (при $N=1$ ) или пары функций Арфа (при $N=2$ ). Компоненты связности пространств голоморфных отображений определяются классами сопряженности подгрупп свободной группы. Компоненты связности пространств суперголоморфных отображений определяются классом эквивалентности пары (подгруппа свободной группы, функция Арфа).

В подходе Фрике и Клейна [18] пространство $T$ возникает как пространство классов сопряженности специальных базисов фуксовых групп. Группа Mod возникает при этом как группа замены базисов. Значительно позже [19] Тайхмюллер предложил другое описание пространства $T$ как пространства экстремальных квазиконформных отображений римановых поверхностей. Поэтому в нашем обзоре это пространство $T$ называется пространством Фрике-Клейна-Тайхмюллера.

Существует много методов параметризации пространства Фрике-Клейна-Тайх- 
мюллера. Большинство из них, как и в оригинальной работе Фрике и Клейна, использует параметры, связанные со стандартной метрикой постоянной кривизны римановой поверхности: длины геодезических, отвечающих образующим фуксовой группы, длины геодезических, отвечаюших стандартным произведениям образуюших, и углы меж ду ними (см., например, [20]-[22]).

Мы используем параметры [23], описьваюшие образуюшие фуксовой групшы через их неподвижные точки и "параметры" сдвигов. Такой подходпозволяет легко перейти от описания модулей римановых поверхностей к описанию пространств голоморффных отображений и после некоторой модификации к описанию супераналогов этих пространств. Мы явно указываем область значений этих параметров для стандартных образующих фуксовых групп. Она оказьвается областью линейного пространства (или его супераналога), выделенной линейными и квадратичными неравенствами. Отсюда легко доказьвается ее гомеоморфность (супер)линейному пространству.

Подчеркнем, что "параметры" сдвигов и их супераналоги - это именно те параметры, которые участвуют в формулах для меры Мамфорда и ее супераналогов [2], [4]. Таким образом, наша параметризация пространства модулей может оказаться удобной для теории струн. Описанный подход оказьвается удобным и при описании вещественных алгебраических кривых и их супераналогов [23]-[25]. Он позволяет, в частности, решить проблему Шоттки для вещественных алгебраических кривых и описать физически важные решения уравнений Кадомцева-Петвиашвили, построить квазипериодический двумерный оператор Шрёдингера и др. [26], [27]. Но эта тема, как нам кажется, заслуживает отдельного обзора.

\section{§ 1. Фуксовы группы и их последовательные образуюшие}

Напомним, что верхняя полуплоскость

$$
\Lambda=\{z \in \mathbb{C} \mid \operatorname{Im} z>0\}
$$

обладает метрикой Лобачевского постоянной отрицательной кривизны $d s=\frac{|d z|}{\operatorname{Im} z}$. Ее геодезические - это полуокружности и полупрямые, ортогональные $\mathbb{R}$. Групша голоморфных автоморфизмов $\operatorname{Aut}(\Lambda) \cong \operatorname{PSL}(2, \mathbb{R})$ совпадает с группой сохраняющих ориентацию изометрий метрики Лобачевского.

Множество Aut $(\Lambda) \backslash$ id распадается на подмножества эллиптических $\operatorname{Aut}_{0}(\Lambda)$, параболических $\operatorname{Aut}_{1}(\Lambda)$ и гиперболических $\operatorname{Aut}_{2}(\Lambda)$ автоморфизмов, имеюших соответственно 0,1 и 2 неподвижные точки на абсолюте $\partial \Lambda=\mathbb{R} \cup \infty$.

Параболический автоморфизм с конечной неподвижной точкой $a \in \mathbb{R}$ имеет вид

$$
C(z)=\frac{(1-a \gamma) z+a^{2} \gamma}{-\gamma z+(1+a \gamma)}
$$

где $\gamma$ называется параметром сдвига. Автоморфизм $C$ назовем положительныцм, если $\gamma>0$. В этом случае $C(r)>r$ при $r \in \mathbb{R} \backslash a$. На верхней полуплоскости автоморфизм $C$ удобно изображать как на рис. 1.1, где $\ell(C)=a$.

Гиперболический автоморфизм с конечной неподвижной притягиваюшей точкой $\alpha$ и конечной неподвижной отталкивающей точкой $\beta$ имеет вид

$$
C(z)=\frac{(\lambda \alpha-\beta) z+(1-\lambda) \alpha \beta}{(\lambda-1) z+(\alpha-\lambda \beta)},
$$




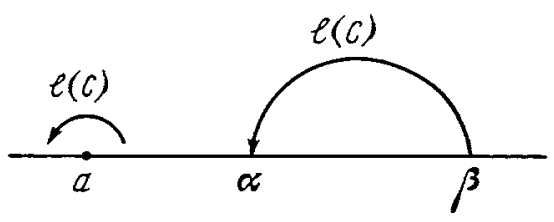

Рис. 1.1

где $\lambda>1$ назьвается параметром сдвига. Автоморфизм $C$ назовем положительным. , если $\alpha<\beta$. В этом случае $C(r)>r$ при $r \in \mathbb{R} \backslash[\alpha, \beta]$ и $C(r)<r$ при $r \in(\alpha, \beta)$. На верхней полуплоскости автоморфизм $C$ удобно изображать как на рис. 1.1, где $\ell(C)$ - геодезическая на $\Lambda$.

Для автоморфизмов $C_{1}, C_{2}$ с конечными неподвижными точками на $\mathbb{R}$ положим $C_{1}<C_{2}$, если все неподвижные точки $C_{1}$ меньше любой неподвижной точки $C_{2}$. Набор $\left\{C_{1}, C_{2}, C_{3}\right\} \subset \operatorname{Aut}_{1}(\Lambda) \cup \operatorname{Aut}_{2}(\Lambda)$ назовем упорядоченным, если $C_{1} \cdot C_{2} \cdot C_{3}=1$ и сушествует $D \in \operatorname{Aut}(\Lambda)$ такой, что автоморфизмы $\widetilde{C}_{i}=D C_{i} D^{-1}(i=1,2,3)$ имеют конечные неподвижные точки, положительны и $\widetilde{C}_{1}<\widetilde{C}_{2}<\widetilde{C}_{3}$. Упорядоченньй набор $\left\{C_{1}, C_{2}, C_{3}\right\}$ назовем последовательным, если $\left\{C_{1} C_{2} C_{1}^{-1}, C_{1}, C_{3}\right\}$ также упорядоченный набор. Набор $\left\{C_{1}, \ldots, C_{n}\right\}$ назовем последовательным типа $(0, k, m)$, если для любого $i=1, \ldots, n-1$ наборы $\left\{C_{1} \cdots C_{i-1}, C_{i}, C_{i+1} \cdots C_{n}\right\}$ последовательные, причем $n=k+m, C_{i} \in \operatorname{Aut}_{2}(\Lambda)$ при $i \leqslant k$ и $C_{i} \in \operatorname{Aut}_{1}(\Lambda)$ при $i>k$.

Для геометрических рассуждений удобно перейти от верхней полуплоскости $\Lambda \mathrm{k}$ внутренности круга $H=\{z \in \mathbb{C}|| z \mid<1\}$. Группа ее голоморфных автоморфизмов $\operatorname{Aut}(H)$ также распадается на эллиптические $\left(\operatorname{Aut}_{0}(H)\right)$, параболические $\left(\operatorname{Aut}_{1}(H)\right)$, гиперболические $\left(\operatorname{Aut}_{2}(H)\right)$ автоморфизмы, имеющие соответственно 0, 1, 2 неподвижные точки на абсолюте $\partial H=\{z \in \mathbb{C}|| z \mid=1\}$. Биголоморфный изоморфизм $\varphi: \Lambda \rightarrow H$ переводит метрику на $\Lambda$ в инвариантную относительно $\operatorname{Aut}(H)$ метрику на $H$, в которой геодезические - это дуги окружностей, ортогональные $\partial H$. При этом

$$
\operatorname{Aut}_{i}(H)=\varphi \operatorname{Aut}_{i}(\Lambda) \varphi^{-1}
$$

Для $C \in \operatorname{Aut}_{1}(H) \cup \operatorname{Aut}_{2}(H)$ положим $\ell(C)=\varphi\left(\ell\left(\varphi^{-1} C \varphi\right)\right)$. Набор $\left\{C_{1}, \ldots, C_{n}\right\} \in$ $\operatorname{Aut}(H)$ назовем последовательным , если $\left\{\varphi^{-1} C_{1} \varphi, \ldots, \varphi^{-1} C_{n} \varphi\right\} \in \operatorname{Aut}(\Lambda)$ - последовательный набор. Согласно нашим определениям, ему отвечает рис. 1.2 , где $\ell=C_{j} C_{j+1} \cdots C_{n-1} C_{n}\left(\ell\left(C_{1}\right)\right)$ лежит между $\ell\left(C_{j-1}\right)$ и $\ell\left(C_{j}\right)$.

Напомним, что дискретная подгруппа группы $\operatorname{Aut}(H)$ или $\operatorname{Aut}(\Lambda)$ назьвается фуксовой группой. Мы будем рассматривать только фуксовы группы без эллиптических элементов. Поэтому далее под фуксовой группой мы понимаем дискретные подгруппы $\operatorname{Aut}(H)$ или $\operatorname{Aut}(\Lambda)$ без эллиптических элементов.

Лемма 1.1. Последовательный набор $V=\left\{C_{1}, \ldots, C_{n}\right\} \subset \operatorname{Aut}(H)$ muna $(0, k, m)$ порождает фуксову группу Г. При этом $H / \Gamma-$ сфера с $k$ дырами и проколами.

ДокаЗАТЕЛЬСтво. Пусть сначала $k>0$. Рассмотрим точку $O_{1} \subset \ell\left(C_{1}\right)$ и положим $O_{i}=C_{i} C_{i+1} \cdots C_{n}\left(O_{1}\right)$ (см. рис. 1.2). Пусть $r_{i}$ - пересекающий $\ell\left(C_{i}\right)$ геодезический луч с началом в точке $O_{i}$ и концами в точке абсолюта. Тогда $d_{i}=C_{i}^{-1} r_{i}-$ 


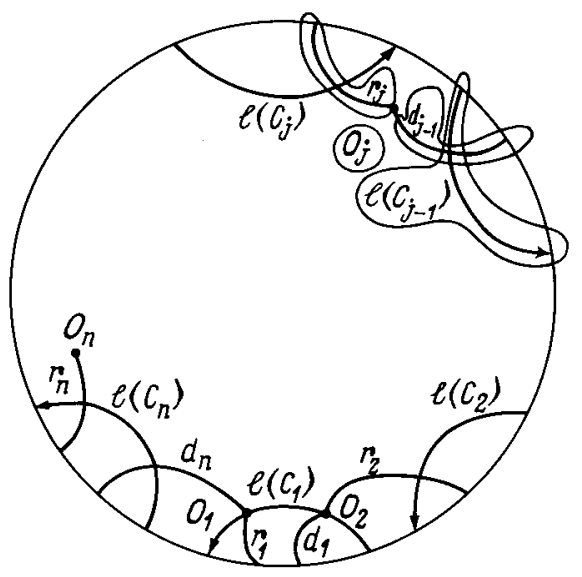

Рис. 1.2

геодезический луч с концом $O_{i+1}$. Лучи $\left\{r_{i}, d_{i}(i=1, \ldots, n)\right\}$ ограничивают некомпактную область $M$. Наше определение гарантирует, что последовательность многоугольников

$$
M, C_{1} M, C_{1} C_{2} M, \ldots, C_{1} \cdots C_{n-1} M
$$

реализует однократный обход вокруг точки $O_{1}$. Согласно [28] отсюда следует, что $M$ - фундаментальная область для порожденной $\left\{C_{1}, \ldots, C_{n}\right\}$ группы $\Gamma$. Автоморфизм $C_{i}$ отождествляет $r_{i}$ и $d_{i}$, образуя на поверхности $H / \Gamma$ дыру при $i \leqslant k$ и прокол при $i>k$. Эта конструкция годится и в случае $k=0$, если в качестве точки $O_{1}$ взять точку, достаточно близкую к неподвижной точке параболического автоморфизма $C_{1}$.

Последовательным набором типа $(g, k, m)$ назовем набор

$$
\left\{A_{1}, B_{1}, \ldots, A_{g}, B_{g}, C_{1}, \ldots, C_{n}\right\}
$$

такой, что $A_{i}, B_{i}(i=1, \ldots, g)$ - гиперболические автоморфизмы и

$$
\left\{A_{1}, B_{1} A_{1}^{-1} B_{1}^{-1}, \ldots, A_{g}, B_{g} A_{g}^{-1} B_{g}^{-1}, C_{1}, \ldots, C_{n}\right\}
$$

- последовательньй набор типа $(0,2 g+k, m)$.

Поверхность рода $g$ с $k$ дырами и $m$ проколами назовем поверхностью типа $(g, k, m)$. Будем говорить, что фуксова групnа $\Gamma \subset \operatorname{Aut}(H)$ (соответственно $\Gamma \subset \operatorname{Aut}(\Lambda))$ uмeеm mun $(g, k, m)$, если $H / \Gamma$ (соответственно $\Lambda / \Gamma)$ - поверхность типа $(g, k, m)$.

ТЕорема 1.1 [21], [29]. Последовательный набор типа $(g, k, m)$ порожсдает фуксову группу $\Gamma$ muna $(g, k, m)$. 


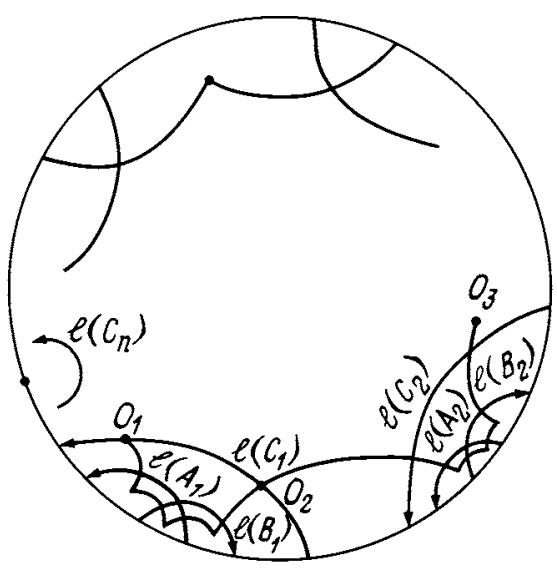

Рис. 1.3

ДокаЗАТельСтво. Пусть $\left\{A_{1}, B_{1}, \ldots, A_{g}, B_{g}, C_{g+1}, \ldots, C_{n}\right\} \in \operatorname{Aut}(H)$ - последовательный набор типа $(g, m, k)$. При $g=0$ утверждение следует из леммы 1.1. Пусть $g>0$. Положим $C_{i}=\left[A_{i} B_{i}\right](i=1, \ldots, g)$. Наши определения гарантируют расположение геодезических $\ell\left(A_{i}\right), \ell\left(B_{i}\right), \ell\left(C_{i}\right)$, показанное на рис. 1.3. Пусть $O_{1} \in \ell\left(C_{1}\right)$ и $M-$ многоугольник, построенньй при доказательстве леммы 1.1.

При $i \leqslant g$ заменим лучи $r_{i}, d_{i}$ на геодезические сегменты с вершинами

$$
O_{i}, A_{i} B_{i}^{-1} A_{i}^{-1} O_{i}, B_{i}^{-1} A_{i}^{-1} O_{i}, A_{i}^{-1} O_{i}, B_{i} A_{i} B_{i}^{-1} A_{i}^{-1} O_{i}=O_{i+1} .
$$

В результате получим новый многоугольник $\widetilde{M}$ (см. рис. 1.3). Наши определения гарантируют, что многоугольники

$$
\begin{gathered}
\widetilde{M}, A_{1} \widetilde{M}, A_{1} B_{1} \widetilde{M}, A_{1} B_{1} A_{1}^{-1} \widetilde{M}, C_{1} \widetilde{M}, C_{1} A_{2} \widetilde{M}, C_{1} A_{2} B_{2} \widetilde{M}, C_{1} A_{2} B_{2} A_{2}^{-1} \widetilde{M}, \\
C_{1} C_{2} \widetilde{M}, \ldots, C_{1} C_{2} \cdots C_{n-1} M
\end{gathered}
$$

реализуют обход вокруг точки $O_{1}$ и, следовательно, [28] множество $\left\{A_{1}, \ldots, C_{n}\right\}$ порождает фуксову группу $\Gamma$. Легко проследить, что каждая пара $\left(A_{i}, B_{i}\right)$ порождает ручку поверхности $H / \Gamma$. Поэтому $H / \Gamma-$ поверхность типа $(g, k, m)$.

\section{§2. Геометрия фуксовых групп}

Пусть $P$ - поверхность типа $(g, k, m)$. Систему образующих

$$
v=\left\{a_{i}, b_{i}(i=1, \ldots, g), c_{i}(i=g+1, \ldots, n)\right\}
$$

группы $\pi_{1}(P, p)$ назовем стандартной, если $v$ порождает $\pi_{1}(P, p)$ с определяюшим соотношением

$$
\prod_{i=1}^{g}\left[a_{i}, b_{i}\right] \prod_{i=g+1}^{n} c_{i}=1
$$


и представляется набором простых контуров

$$
\widetilde{v}=\left\{\widetilde{a}_{i}, \widetilde{b}_{i}(i=1, \ldots, g), \widetilde{c}_{i}(i=g+1, \ldots, n)\right\}
$$

со следуюшими свойствами:

1) контур $\widetilde{c}_{i}$ гомологичен 0 и отсекает от поверхности $P$ одну дыру при $i \leqslant g+k$ и один прокол при $i>g+k$;

2) $\widetilde{a}_{i} \cap \widetilde{b}_{j}=\widetilde{a}_{i} \cap \widetilde{c}_{j}=\widetilde{b}_{i} \cap \widetilde{c}_{j}=\widetilde{c}_{i} \cap \widetilde{c}_{j}=p$;

$3)$ в окрестности точки $p$ контуры $\widetilde{v}$ расположены как на рис. 2.1.

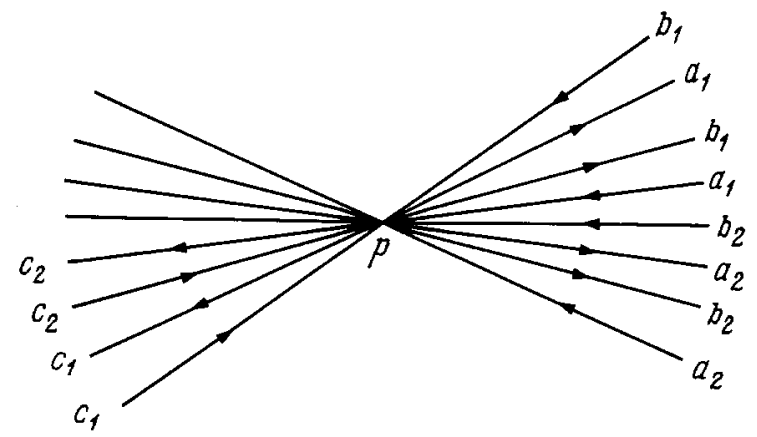

Рис. 2.1

В этом случае набор контуров $\widetilde{v}$ расположен на $P$ как показано на рис. 2.2.

Пусть теперь $\Gamma \subset \operatorname{Aut}(H)$ - фуксова группа, $P=H / \Gamma, \Phi: H \rightarrow P$ - естественная проекция и $q \in \Phi^{-1}(p)$. Сопоставим автоморфизму $C \in \Gamma$ геодезический отрезок $\ell_{q}(C) \subset H$ с конщами $q$ и $C(q)$. Соответствие $C \mapsto \Phi\left(\ell_{q}(C)\right)$ порождает изоморфизм $\Phi_{q}: \Gamma \rightarrow \pi_{1}(P, p)$.

Лемма 2.1. Пусть $V=\left\{A_{i}, B_{i}(i=1, \ldots, g), C_{i}(i=g+1, \ldots, n)\right\}-$ последовательный набор типа $(g, k, m), \Gamma$ - фуксова группа, которую он порохсдат, $u$ $P=H / \Gamma$. Тогда $v_{q}=\Phi_{q}(V)$ - стандартная система образующих группьи $\pi_{1}(P, p)$.

ДокАЗАТЕЛЬСТво. Рассмотрим фундаментальную область $M$, построенную при доказательстве леммы 1.1 и теоремы 1.1 (см. рис. 1.3 ). При $i>g$ соединим точки $O_{i}$ и $O_{i+1}$ попарно непересекающимися отрезками $c_{i} \subset M$. (Здесь $O_{n+1}=O_{1}$.) Рассмотрим на $H$ геодезические сегменты

$$
a_{i}=\left[O_{i}, A_{i} B_{i}^{-1} A_{i}^{-1} O_{i}\right], \quad b_{i}=\left[A_{i} B_{i}^{-1} A_{i}^{-1} O_{i}, B_{i}^{-1} A_{i}^{-1} O_{i}\right] .
$$

Тогда естественная проекция $\Phi: H \rightarrow P$ порождает стандартную систему образующих

$$
V_{O_{1}}=\left\{\Phi\left(a_{i}\right), \Phi\left(b_{i}\right)(i=1, \ldots, g), \Phi\left(c_{i}\right)(i=g+1, \ldots, n)\right\} \in \pi_{1}\left(P, \Phi\left(O_{1}\right)\right) .
$$

Непрерывный перенос точки $O_{1}$ в $q$ переводит $V_{O_{1}}$ в стандартную систему образующих $V_{q}$.

Цель этого параграфа - доказать обрашение этой леммы. 


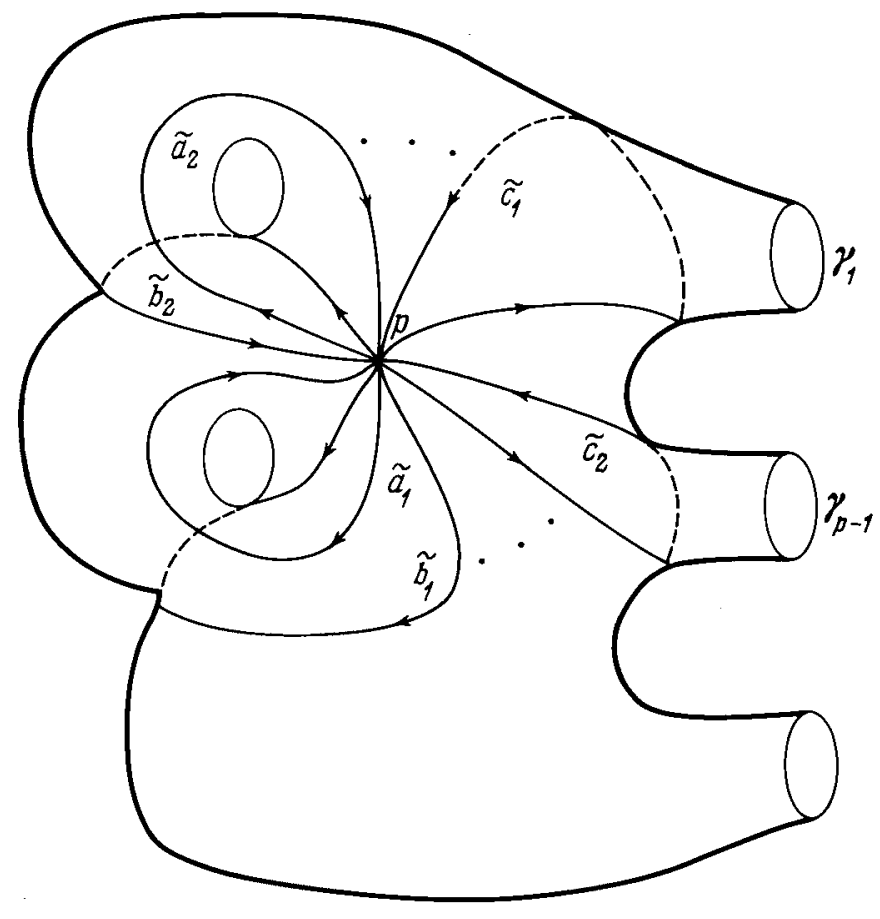

Рис. 2.2

TeOpema 2.1 [21], [29], [30]. Пусть $\Gamma \subset \operatorname{Aut}(H)$ - фуксова грynna muna $(g, k, m)$, $P=H / \Gamma, \Phi: H \rightarrow P-$ естественная проекиия $и$

$$
v=\left\{a_{i}, b_{i}(i=1, \ldots, g), c_{i}(i=g+1, \ldots, n)\right\}
$$

- стандартная система образующих группь $\pi_{1}(P, \Phi(q))$. Тогда $V=\Phi_{q}^{-1}(v)-$ последовательный набор типа $(g, k, m)$.

Для доказательства, основанного на [29], нам понадобится несколько дополнительных определений и лемм.

Пусть $\widetilde{a}$ - контур, представляющий $a \in \pi_{1}(P, \Phi(q))$. Будем обходить контур $\widetilde{a}$, начиная с точки $\Phi(q)$, “поднимая" этот обход на $H$, начиная с точки $q$. После бесконечного числа обходов в обоих направлениях мы получим линию $\ell(\widetilde{a}) \subset M$ с концами в неподвижных точках автоморфизма $A=\Phi_{q}^{-1}(a)$ (см. рис. 2.3).

ЛЕмма 2.2. Если $\widetilde{a}$ не имеет самопересечений, то $h \ell(A)$ и $\ell(A)$ не пересекаются при всех $h \in \Gamma$.

ДокАЗАТЕльство. Пусть $h \ell(A)$ и $\ell(A)$ пересекаются при $h \in \Gamma$. Тогда $h \ell(\widetilde{a})$ и $\ell(\widetilde{a})$ также пересекаются (см. рис. 2.3).

ЛЕмма 2.3. Пусть контуры $\widetilde{a}, \widetilde{b}$ представляют әлементы $a, b \in \pi_{1}(P, \Phi(q))$. Пусть существует малая деформащия контуров $\widetilde{a}$ u $\widetilde{b}$, переводящая их в непересекающиеся контуры. Тогда

$$
\ell\left(\Phi_{q}^{-1}(a)\right) \cap \ell\left(\Phi_{q}^{-1}(b)\right)=\varnothing .
$$




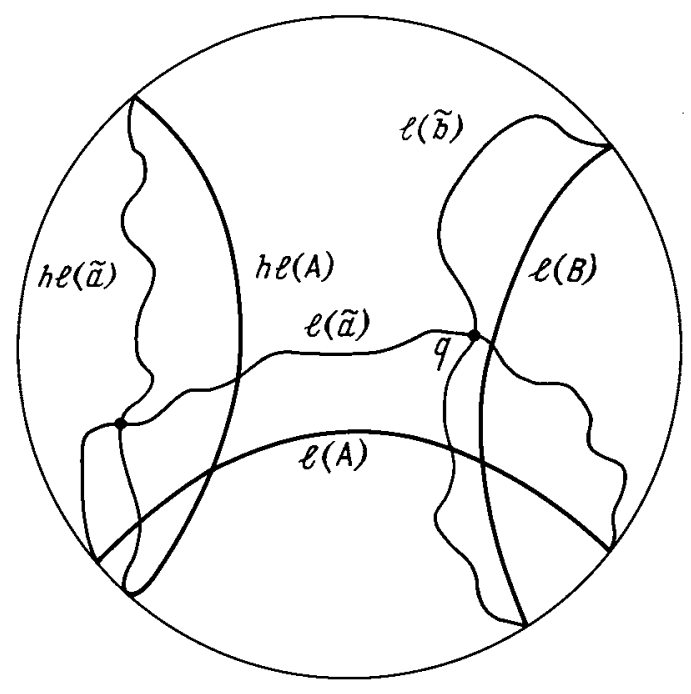

Рис. 2.3

ДОкАЗАТЕЛЬСТвО. Если

$$
\ell\left(\Phi_{q}^{-1}(a)\right) \cap \ell\left(\Phi_{q}^{-1}(b)\right) \neq \varnothing,
$$

то $\ell(\widetilde{a})$ и $\ell(\widetilde{b})$ пересекаются так, что их пересечение нельзя исключить малой деформацией (см. рис. 2.3).

ЛЕмма 2.4. Пусть контурь $\widetilde{c}_{1}, \widetilde{c}_{2}, \widetilde{c}_{3}$ не имеют самопересечений и представляют $c_{1}, c_{2}, c_{3} \in \pi_{1}(P, \Phi(q))$ такие, что $c_{1} \cdot c_{2} \cdot c_{3}=1$. Пусть существует малая деформачия контуров $\widetilde{c}_{1}, \widetilde{c}_{2}$ и $\widetilde{c}_{3}$, переводящая их в попарно непересекающиеся контуры. Тогда или набор

$$
\left\{\Phi_{q}^{-1}\left(c_{1}\right), \Phi_{q}^{-1}\left(c_{2}\right), \Phi_{q}^{-1}\left(c_{3}\right)\right\}
$$

или набор

$$
\left\{\Phi_{q}^{-1}\left(c_{3}^{-1}\right), \Phi_{q}^{-1}\left(c_{2}^{-1}\right), \Phi_{q}^{-1}\left(c_{1}^{-1}\right)\right\}
$$

является последовательныл.

ДокАЗАТЕльСтво. Положим $C_{i}=\Phi_{q}^{-1}\left(c_{i}\right)$. Согласно лемме $2.3 \ell\left(C_{1}\right) \cap \ell\left(C_{2}\right)=\varnothing$. Предположим, что $\ell\left(C_{1}\right)$ и $\ell\left(C_{2}\right)$ расположены как на рис. 2.4 .

Рассмотрим дуги $\alpha=\left(\alpha_{1}, \alpha_{2}\right)$ и $\beta=\left(\beta_{1}, \beta_{2}\right)$ на $\partial H$. Тогда $C_{1} \alpha \subset \alpha, C_{2} \alpha \subset \alpha$, откуда $C_{3}^{-1} \alpha=C_{1} C_{2} \alpha \subset \alpha$. Аналогично $C_{3}^{-1} \beta \subset \beta$. Таким образом, $\ell\left(C_{3}\right)$ проходит так, как показано пунктиром на рис. 2.4. Но тогда согласно лемме $2.2 C_{1} \ell\left(C_{3}\right) \subset N_{1}$ и, следовательно, $C_{1} \beta_{2} \in N_{1}$. Это невозможно, поскольку

$$
C_{1} \beta_{2}=C_{3}^{-1} C_{2}^{-1} \beta_{2}=C_{3}^{-1} \beta \subset N_{2} .
$$

Таким образом, $\ell\left(C_{1}\right)$ и $\ell\left(C_{2}\right)$ расположены как на рис. 2.5 или рис. 2.6. 


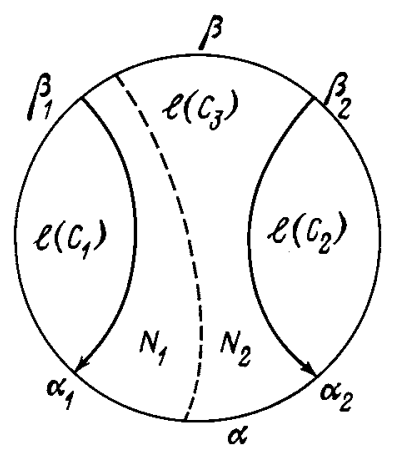

Рис. 2.4

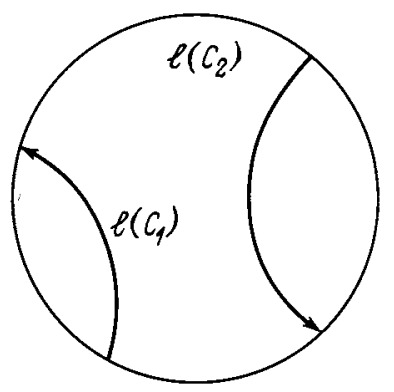

Рис. 2.5

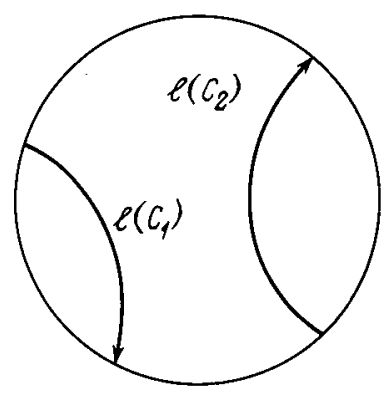

Рис. 2.6

Аналогичное утверждение верно также для пар $\left(C_{2}, C_{3}\right)$ и $\left(C_{3}, C_{1}\right)$. Отсюда следует, что или набор $\left\{C_{1}, C_{2}, C_{3}\right\}$, или набор $\left\{C_{3}^{-1}, C_{2}^{-1}, C_{1}^{-1}\right\}$ является упорядоченным в смысле $\S 1$.

Аналогично, упорядоченными являются наборы $\left\{C_{1} C_{2} C_{1}^{-1}, C_{1}, C_{3}\right\}$ или наборы $\left\{C_{3}^{-1}, C_{1}^{-1}, C_{1} C_{2}^{-1} C_{1}^{-1}\right\}$. Среди наборов $\left\{C_{1}, C_{2}, C_{3}\right\}$ и $\left\{C_{3}^{-1}, C_{1}^{-1}, C_{1} C_{2}^{-1} C_{1}^{-1}\right\}$ упорядочен может быть только один. Таким образом, или наборы $\left\{C_{1}, C_{2}, C_{3}\right\}$, $\left\{C_{1} C_{2} C_{1}^{-1}, C_{1}, C_{3}\right\}$, или наборы $\left\{C_{3}^{-1}, C_{2}^{-1}, C_{1}^{-1}\right\},\left\{C_{3}^{-1}, C_{1}^{-1}, C_{1} C_{2}^{-1} c_{1}^{-1}\right\}$ являются упорядоченнњгми.

ДОКАЗАТЕЛЬСТво ТЕОРЕМЫ 2.1. ПолоЖим $A_{i}=\Phi_{q}^{-1}\left(a_{i}\right), B_{i}=\Phi_{q}^{-1}\left(b_{i}\right), C_{i}=$ 
$\Phi_{q}^{-1}\left(c_{i}\right)$. Рассмотрим наборы

$$
\left\{x_{1}, \ldots, x_{g+n}\right\}=\left\{a_{1}, b_{1} a_{1}^{-1} b_{1}^{-1}, \ldots, a_{g}, b_{g} a_{g}^{-1} b_{g}^{-1}, c_{g+1}, \ldots, c_{n}\right\}
$$

и

$$
\left\{X_{1}, \ldots, X_{g+n}\right\}=\left\{A_{1}, B_{1} A_{1}^{-1} B_{1}^{-1}, \ldots, A_{g}, B_{g} A_{g}^{-1} B_{g}^{-1}, C_{g+1}, \ldots, C_{n}\right\} .
$$

Применяя лемму 2.4 к наборам

$$
\left\{x_{1} \cdots x_{\ell-1}, x_{\ell}, x_{\ell+1} \cdots x_{g+n}\right\}
$$

находим, что или все наборы вида

$$
\left\{X_{1} \cdots X_{\ell-1}, X_{\ell}, X_{\ell+1} \cdots X_{g+n}\right\}
$$

или все наборы вида

$$
\left\{X_{g+n}^{-1} \cdots X_{\ell+1}^{-1}, X_{\ell}^{-1}, X_{\ell-1}^{-1} \cdots X_{1}^{-1}\right\}
$$

являются последовательными. То есть или набор $\left\{X_{1}, \ldots, X_{g+n}\right\}$, или набор $\left\{X_{g+n}^{-1}, \ldots, X_{1}^{-1}\right\}$ являетсяпоследовательным. В последнем случае согласно лемме 2.1 контуры, представляюшие $a_{i}, b_{i}, c_{i}$, расположены не так, как на рис. 2.1, и, следовательно, именно набор $\left\{X_{1}, \ldots, X_{g+n}\right\}$ является последовательным набором типа $(0,2 g+k, m)$. Значит, набор

$$
\left\{A_{i}, B_{i}(i=1, \ldots, g), C_{j}(j=g+1, \ldots, n)\right\}
$$

является последовательным, типа $(g, k, m)$.

\section{§3. Свободные фуксовы группы ранга 2}

Согласно теоремам 1.1 и 2.1 всякий последовательньй набор типа $(g, k, m)$ порождает фуксову группу типа $(g, k, m)$ и всякая фуксова группа типа $(g, k, m)$ порождается последовательным набором. В этом параграфе мы найдем все (с точностью до сопряженности в $\operatorname{Aut}(\Lambda))$ последовательные наборы типов $(0,3,0),(0,2,1),(0,1,2)$ и $(1,1,0)$, что дает описания всех фуксовых групп ранга 2.

Лемма 3.1. Всякий упорядоченный набор $\left\{C_{1}, C_{2}, C_{3}\right\}$ является последовательныцм.

ДокАЗАТЕЛЬСТво. Неподвижные точки сдвигов $C_{i}$ разбивают абсолют на 6 дуг $\gamma_{i}$, изображенных на рис. 3.1 .

Пусть $\alpha$ и $\beta$ - неподвижные притягиваюшая и отталкиваюшая точки сдвига $C_{1} C_{2} C_{1}^{-1}$. Тогда

$$
\ell\left(C_{1} C_{2} C_{1}^{-1}\right)=C_{1} \ell\left(C_{2}\right) \text { и } \beta \in \gamma_{4} \cup \gamma_{5} \cup \gamma_{6} .
$$

С другой стороны,

$$
C_{1} \ell\left(C_{2}\right)=C_{3}^{-1} C_{2}^{-1} \ell\left(C_{2}\right)=C_{3}^{-1} \ell\left(C_{2}\right) \text { и } \beta \in \gamma_{3} \cup \gamma_{2} \cup \gamma_{1} \cup \gamma_{6} .
$$




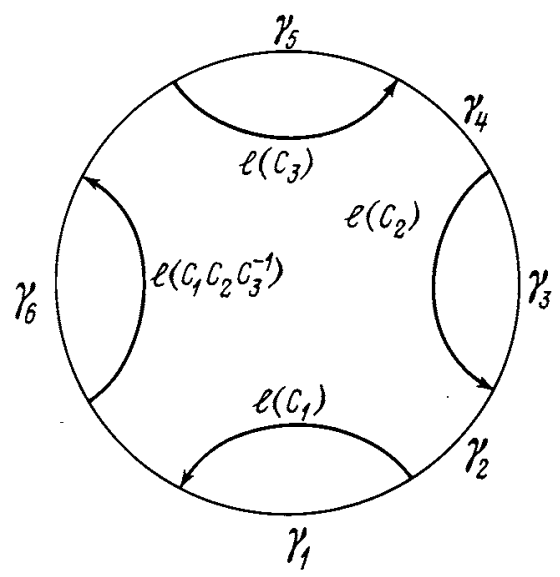

Рис. 3.1

Таким образом, $\beta \in \gamma_{6}$. Аналогично, $\alpha \in \gamma_{6}$. То есть $\left\{C_{1} C_{2} C_{1}^{-1}, C_{1}, C_{3}\right\}$ также упорядоченный набор.

Лемма 3.2 [23]. Пусть

$C_{1} z=\lambda_{1} z\left(\lambda_{1}>1\right), \quad C_{2}(z)=\frac{\left(\lambda_{2} \alpha-\beta\right) z+\left(1-\lambda_{2}\right) \alpha \beta}{\left(\lambda_{2}-1\right) z+\left(\alpha-\lambda_{2} \beta\right)}\left(\lambda_{2}>1\right), \quad C_{3}=\left(C_{1} C_{2}\right)^{-1}$.

Тогда $\left\{C_{1}, C_{2}, C_{3}\right\}$ - последовательный набор, если и только если

$$
0<\left(\frac{\sqrt{\lambda_{1}}+\sqrt{\lambda_{2}}}{1+\sqrt{\lambda_{1} \lambda_{2}}}\right)^{2} \beta \leqslant \alpha<\beta<\infty
$$

При этом $C_{3} \in \operatorname{Aut}_{1}(\Lambda)$, если и только если

$$
\left(\frac{\sqrt{\lambda_{1}}+\sqrt{\lambda_{2}}}{1+\sqrt{\lambda_{1} \lambda_{2}}}\right)^{2} \beta=\alpha
$$

ДокАЗАТЕЛЬСТво. По условию

$$
C_{3}^{-1}(z)=\lambda_{1} \frac{\left(\lambda_{2} \alpha-\beta\right) z+\left(1-\lambda_{2}\right) \alpha \beta}{\left(\lambda_{2}-1\right) z+\left(\alpha-\lambda_{2} \beta\right)} .
$$

Неподвижные точки $C_{3}$ - это корни уравнения $C_{3}^{-1}(x)=x$, т.е.

$$
\left(\lambda_{2}-1\right) x^{2}-\left(\lambda_{2} \beta-\alpha-\lambda_{1} \beta+\lambda_{1} \lambda_{2} \alpha\right) x+\lambda_{1}\left(\lambda_{2}-1\right) \alpha \beta=0 .
$$

Следовательно: 1) $C_{3} \in \operatorname{Aut}_{2}(H)$, если и только если

$$
\left(\alpha+\lambda_{1} \lambda_{2} \alpha-\lambda_{1} \beta-\lambda_{2} \beta\right)^{2}-4 \lambda_{1} \lambda_{2}(\beta-\alpha)^{2}>0,
$$


T.e.

$$
\alpha>\left(\frac{\sqrt{\lambda_{1}}+\sqrt{\lambda_{2}}}{1+\sqrt{\lambda_{1} \lambda_{2}}}\right)^{2} \beta \text { или } \alpha<\left(\frac{\sqrt{\lambda_{1}}-\sqrt{\lambda_{2}}}{\sqrt{\lambda_{1} \lambda_{2}}-1}\right)^{2} \beta ;
$$

2) $C_{3} \in \operatorname{Aut}_{1}(H)$, если и только если

$$
\left(\alpha+\lambda_{1} \lambda_{2} \alpha-\lambda_{1} \beta-\lambda_{2} \beta\right)^{2}-4 \lambda_{1} \lambda_{2}(\beta-\alpha)^{2}=0,
$$

T.e.

$$
\alpha=\left(\frac{\sqrt{\lambda_{1}}+\sqrt{\lambda_{2}}}{1+\sqrt{\lambda_{1} \lambda_{2}}}\right)^{2} \beta \text { или } \alpha=\left(\frac{\sqrt{\lambda_{1}}-\sqrt{\lambda_{2}}}{\sqrt{\lambda_{1} \lambda_{2}}-1}\right)^{2} \beta .
$$

Пусть теперь $\left\{C_{1}, C_{2}, C_{3}\right\}$ - последовательный набор и $\bar{\alpha} \leqslant \bar{\beta}$ - корни уравнения (3.2).

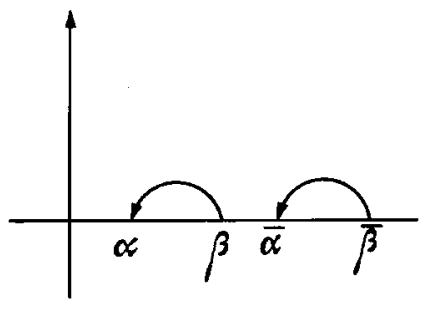

Рис. 3.2

Тогда $0<\alpha<\beta<\bar{\alpha}$ (см. рис. 3.2), откуда

$$
\frac{\lambda_{2} \beta-\alpha-\lambda_{1} \beta+\lambda_{1} \lambda_{2} \alpha}{2\left(\lambda_{2}-1\right)}=\frac{\bar{\alpha}+\bar{\beta}}{2}>\beta .
$$

То есть, ввиду $\lambda_{1} \lambda_{2}-1>\lambda_{1}+\lambda_{2}-2$,

$$
\begin{aligned}
\alpha & >\frac{\lambda_{1}+\lambda_{2}-2}{\lambda_{1} \lambda_{2}-1} \beta>\frac{\lambda_{1}+\lambda_{2}-2+2\left(1-\sqrt{\lambda_{1} \lambda_{2}}\right)}{\lambda_{1} \lambda_{2}-1+2\left(1-\sqrt{\lambda_{1} \lambda_{2}}\right)} \beta \\
& =\frac{\lambda_{1}+\lambda_{2}-2 \sqrt{\lambda_{1} \lambda_{2}}}{\lambda_{1} \lambda_{2}-2 \sqrt{\lambda_{1} \lambda_{2}}+1} \beta=\left(\frac{\sqrt{\lambda_{1}}-\sqrt{\lambda_{2}}}{\sqrt{\lambda_{1} \lambda_{2}}-1}\right)^{2} \beta .
\end{aligned}
$$

Следовательно,

$$
\alpha \geqslant\left(\frac{\sqrt{\lambda_{1}}+\sqrt{\lambda_{2}}}{\sqrt{\lambda_{1} \lambda_{2}}+1}\right)^{2} \beta .
$$

Пусть теперь

$$
0<\left(\frac{\sqrt{\lambda_{1}}+\sqrt{\lambda_{2}}}{\sqrt{\lambda_{1} \lambda_{2}}+1}\right)^{2} \beta \leqslant \alpha<\beta<\infty
$$

и $\bar{\alpha} \leqslant \bar{\beta}$ - корни уравнения (3.2). Соотношение $\beta<\bar{\alpha}$ эквивалентно паре соотношений

$$
\begin{gathered}
\bar{\alpha} \cdot \bar{\beta}>\beta^{2} \\
\left(\lambda_{2}-1\right) \beta^{2}-\left(\lambda_{2} \beta-\alpha-\lambda_{1} \beta+\lambda_{1} \lambda_{2} \alpha\right) \beta+\lambda_{1}\left(\lambda_{2}-1\right) \alpha \beta>0 .
\end{gathered}
$$


Соотношение (3.3) очевидно ввиду

$$
\bar{\alpha} \cdot \bar{\beta}=\lambda_{1} \alpha \beta \geqslant \lambda_{1}\left(\frac{\sqrt{\lambda_{1}}+\sqrt{\lambda_{2}}}{\sqrt{\lambda_{1} \lambda_{2}}+1}\right)^{2} \beta^{2}>\beta^{2} .
$$

Соотношение (3.4) следует из

$\lambda_{2} \beta^{2}-\beta^{2}-\lambda_{2} \beta^{2}+\alpha \beta+\lambda_{1} \beta^{2}-\lambda_{1} \lambda_{2} \alpha \beta+\lambda_{1} \lambda_{2} \alpha \beta-\lambda_{1} \alpha \beta=\left(\lambda_{1}-1\right)\left(\beta^{2}-\alpha \beta\right)>0$.

Таким образом, $\beta<\bar{\alpha}$. Знак $C_{3}$ совпадает со знаком $C_{3}(0)=C_{2}^{-1} C_{1}^{-1}(0)>0$. Следовательно, $\left\{C_{1}, C_{2}, C_{3}\right\}$ - упорядоченньй набор, который согласно лемме 3.1 является последовательным.

ЛЕМма 3.3. Пусть

$$
C_{1}(z)=\lambda z \quad(\lambda>1), \quad C_{2}(z)=\frac{(1-a \gamma) z+a^{2} \gamma}{-\gamma z+(1+a \gamma)}(\gamma>0), \quad C_{3}=\left(C_{1} C_{2}\right)^{-1}
$$

Тогда $\left\{C_{1}, C_{2}, C_{3}\right\}$ - последовательный набор, если и только если аү $\leqslant \frac{\sqrt{\lambda}+1}{\sqrt{\lambda}-1}$. $\Pi р и$ этом $C_{3} \in \operatorname{Aut}_{1}(H)$, если и только если $а \gamma=\frac{\sqrt{\lambda}+1}{\sqrt{\lambda}-1}$.

ДокАЗАТЕЛЬСТво. По условию

$$
C_{3}(z)=\lambda \frac{(1-a \gamma) z+a^{2} \gamma}{-\gamma z+(1+a \gamma)}
$$

Неподвижные точки $C_{3}-$ это корни уравнения

$$
\gamma x^{2}-(1+a \gamma-\lambda+\lambda a \gamma) x+\lambda a^{2} \gamma=0
$$

Следовательно,

1) $C_{3} \in \operatorname{Aut}_{2}(H)$, если и только если

$$
(1+a \gamma-\lambda+\lambda a \gamma)^{2}>4 \lambda a^{2} \gamma^{2}, \text { т.е. } a \gamma>\frac{\sqrt{\lambda}+1}{\sqrt{\lambda}-1},
$$

2) $C_{3} \in \operatorname{Aut}_{1}(H)$, если и только если

$$
(1+a \gamma-\lambda+\lambda a \gamma)^{2}=4 \lambda a^{2} \gamma^{2}, \quad \text { т.е. } a \gamma=\frac{\sqrt{\lambda}+1}{\sqrt{\lambda}-1}
$$

Пусть теперь $a \gamma \geqslant \frac{\sqrt{\lambda}+1}{\sqrt{\lambda}-1}$ и $\bar{\alpha} \leqslant \bar{\beta}$-корни уравнения (3.5). Тогда $\bar{\alpha} \bar{\beta}=\lambda a^{2}>a^{2}$ и $\gamma a^{2}-(1+a \gamma-\lambda+\lambda a \gamma) a+\lambda a^{2} \gamma=a(\lambda-1)>0$. Следовательно, $0<a<\bar{\alpha}$ и $\left\{C_{1}, C_{2}, C_{3}\right\}$ - упорядоченньй набор, которьй согласно лемме 3.1 является последовательным. 
ЛЕмма 3.4 [29]. Пусть

$$
\begin{aligned}
& A(z)=\frac{\left(\lambda_{A} \alpha_{A}-\beta_{A}\right) z+\left(1-\lambda_{A}\right) \alpha_{A} \beta_{A}}{\left(\lambda_{A}-1\right) z+\left(\alpha_{A}-\lambda_{A} \beta_{A}\right)}, \\
& B(z)=\frac{\left(\lambda_{B} \alpha_{B}-\beta_{B}\right) z+\left(1-\lambda_{B}\right) \alpha_{B} \beta_{B}}{\left(\lambda_{B}-1\right) z+\left(\alpha_{B}-\lambda_{B} \beta_{B}\right)}
\end{aligned}
$$

$u$

$$
C^{-1}=[A, B](z)=\lambda z \quad\left(\lambda_{A}, \lambda_{B}, \lambda>1\right) .
$$

Тогда $\{A, B, C\}$ является последовательныцм набором типа $(1,1,0)$, если и только если

$$
\begin{gathered}
-\infty<\alpha_{A}<\beta_{B}<\beta_{A}<\alpha_{B}<0, \\
\frac{\alpha_{A}}{\beta_{A}}<\sqrt{\lambda}, \quad \frac{\beta_{B}}{\alpha_{B}}<\sqrt{\lambda}, \\
\lambda_{A}=\frac{\alpha_{A} \sqrt{\lambda}-\beta_{A}}{\beta_{A} \sqrt{\lambda}-\alpha_{A}}, \quad \lambda_{B}=\frac{\beta_{B} \sqrt{\lambda}-\alpha_{B}}{\alpha_{B} \sqrt{\lambda}-\beta_{B}}, \\
\alpha_{B} \beta_{B} \lambda-\left[\left(\alpha_{A}+\beta_{A}\right)\left(\alpha_{B}+\beta_{B}\right)-\alpha_{A} \beta_{A}-\alpha_{B} \beta_{B}\right] \sqrt{\lambda}+\alpha_{A} \beta_{A}=0,
\end{gathered}
$$

причем в этом случае

$$
\begin{aligned}
& A(z)=\frac{\left(\alpha_{A}+\beta_{A}\right) \sqrt{\lambda} z-\alpha_{A} \beta_{A}(\sqrt{\lambda}+1)}{(\sqrt{\lambda}+1) z-\left(\alpha_{A}+\beta_{A}\right)}, \\
& B(z)=\frac{\left(\alpha_{B}+\beta_{B}\right) z-\alpha_{B} \beta_{B}(\sqrt{\lambda}+1)}{(\sqrt{\lambda}+1) z-\left(\alpha_{B}+\beta_{B}\right) \sqrt{\lambda}} .
\end{aligned}
$$

ДокаЗАТЕЛЬСТво. Пусть $\{A, B, C\}$ - последовательный набор типа $(1,1,0)$. Тогда $\left\{A, B A^{-1} B^{-1}, C\right\}$ - последовательный набор, откуда $-\infty<\alpha_{A}<\beta_{B}<\alpha_{B}<0$ (см. рис. 3.3).

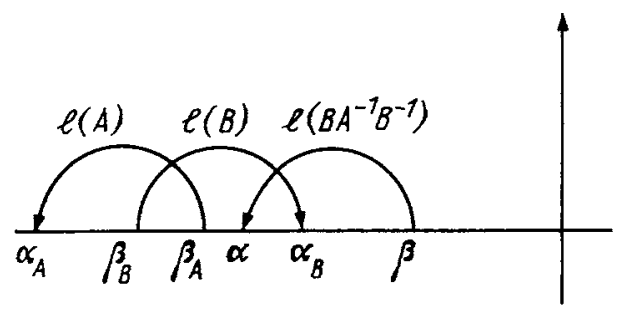

Рис. 3.3 
Рассмотрим $\widetilde{A}=A B A B^{-1} A^{-1}=A C$. Тогда

$$
\frac{\left(\lambda_{A} \alpha_{A}-\beta_{A}\right) \frac{1}{\lambda} z+\left(1-\lambda_{A}\right) \alpha_{A} \beta_{A}}{\left(\lambda_{A}-1\right) \frac{1}{\lambda} z+\left(\alpha_{A}-\lambda_{A} \beta_{A}\right)}=\widetilde{A}(z)=\frac{\left(\lambda_{A} \widetilde{\alpha}-\widetilde{\beta}\right) z+\left(1-\lambda_{A}\right) \widetilde{\alpha} \widetilde{\beta}}{\left(\lambda_{A}-1\right) z+\left(\widetilde{\alpha}-\lambda_{A} \widetilde{\beta}\right)}
$$

(где $\widetilde{\alpha}<\widetilde{\beta}$ - неподвижные точки $\widetilde{A}$ ). Отсюда

$$
\frac{\left(1-\lambda_{A}\right) \alpha_{A} \beta_{A}}{\frac{1}{\lambda}\left(\lambda_{A}-1\right)}=\frac{\left(1-\lambda_{A}\right) \tilde{\alpha} \widetilde{\beta}}{\left(\lambda_{A}-1\right)}
$$

и

$$
\frac{\left(\alpha_{A}-\beta_{A}\right)^{2}}{(\widetilde{\alpha}-\widetilde{\beta})^{2}}=\frac{1}{\lambda}
$$

Первое равенство влечет $\alpha_{A} \beta_{A}=\frac{1}{\lambda} \widetilde{\alpha} \widetilde{\beta}$ и, следовательно, $(\widetilde{\alpha}+\widetilde{\beta})^{2}=(\widetilde{\alpha}-\widetilde{\beta})^{2}+$ $4 \widetilde{\alpha} \widetilde{\beta}=\lambda\left(\alpha_{A}+\beta_{A}\right)^{2}$. Таким образом, равенство

$$
\widetilde{\alpha}+\widetilde{\beta}=\frac{\left(\lambda_{A} \widetilde{\alpha}-\widetilde{\beta}\right)-\left(\widetilde{\alpha}-\lambda_{A} \widetilde{\beta}\right)}{\lambda_{A}-1}=\frac{\frac{1}{\lambda}\left(\lambda_{A} \alpha_{A}-\beta_{A}\right)-\left(\alpha_{A}-\lambda_{A} \beta_{A}\right)}{\frac{1}{\lambda}\left(\lambda_{A}-1\right)}
$$

влечет

$$
\frac{\left(\lambda_{A} \alpha_{A}-\beta_{A}\right)-\lambda\left(\alpha_{A}-\lambda_{A} \beta_{A}\right)}{\left(\lambda_{A}-1\right)}=\sqrt{\lambda}\left(\alpha_{A}+\beta_{A}\right) .
$$

Ввиду $\sqrt{\lambda} \neq 1$ отсюда следует

$$
\sqrt{\lambda}=\frac{\beta_{A}-\alpha_{A} \lambda_{A}}{\alpha_{A}-\beta_{A} \lambda_{A}}>\frac{-\alpha_{A} \lambda_{A}}{-\beta_{A} \lambda_{A}}=\frac{\alpha_{A}}{\beta_{A}} .
$$

т.е. $\lambda_{A}=\frac{\alpha_{A} \sqrt{\lambda}-\beta_{A}}{\beta_{A} \sqrt{\lambda}-\alpha_{A}}$. Аналогично $\lambda_{B}=\frac{\beta_{B} \sqrt{\lambda}-\alpha_{B}}{\alpha_{B} \sqrt{\lambda}-\beta_{B}}$ и $\frac{\beta_{B}}{\alpha_{B}}<\sqrt{\lambda}$. Таким образом,

$$
\begin{aligned}
& A(z)=\frac{\left(\alpha_{A}+\beta_{A}\right) \sqrt{\lambda} z-\alpha_{A} \beta_{A}(\sqrt{\lambda}+1)}{(\sqrt{\lambda}+1) z-\left(\alpha_{A}+\beta_{A}\right)}, \\
& B(z)=\frac{\left(\alpha_{B}+\beta_{B}\right) z-\alpha_{B} \beta_{B}(\sqrt{\lambda}+1)}{(\sqrt{\lambda}+1) z-\left(\alpha_{B}+\beta_{B}\right) \sqrt{\lambda}} .
\end{aligned}
$$

Соотношение $[A, B](z)=\lambda z$ влечет $(3.9)$.

Пусть теперь вьполняется (3.6)-(3.9). Положим

$$
\begin{aligned}
& \widetilde{A}(z)=\frac{\left(\alpha_{A}+\beta_{A}\right) \sqrt{\lambda} z-\alpha_{A} \beta_{A}(\sqrt{\lambda}+1)}{(\sqrt{\lambda}+1) z-\left(\alpha_{A}+\beta_{A}\right)}, \\
& \widetilde{B}(z)=\frac{\left(\alpha_{B}+\beta_{B}\right) z-\alpha_{B} \beta_{B}(\sqrt{\lambda}+1)}{(\sqrt{\lambda}+1) z-\left(\alpha_{B}+\beta_{B}\right) \sqrt{\lambda}} .
\end{aligned}
$$

Тогда $\widetilde{A}\left(\alpha_{A}\right)=\alpha_{A}, \widetilde{A}\left(\beta_{A}\right)=\beta_{\widetilde{A}}, \widetilde{B}\left(\alpha_{B}\right)=\alpha_{B}, \widetilde{B}\left(\beta_{B}\right)=\beta_{B}$. Согласно (3.9) $[\widetilde{A}, \widetilde{B}]=C^{-1}$. Поэтому $\left\{\widetilde{A}, \widetilde{B} \widetilde{A}^{-1} \widetilde{B}^{-1}, C\right\}$ - упорядоченный набор, который согласно 
лемме 3.1 является последовательным. Таким образом, $\{\widetilde{A}, \widetilde{B}, C\}$-последовательньй набор типа $(1,1,0)$. Как уже доказано,

где

$$
\widetilde{A} z=\frac{\left(\widetilde{\lambda}_{A} \alpha_{A}-\beta_{A}\right) z+\left(1-\widetilde{\lambda}_{A}\right) \alpha_{A} \beta_{A}}{\left(\widetilde{\lambda}_{A}-1\right) z+\left(\alpha_{A}-\lambda_{A} \beta_{A}\right)}
$$

$$
\tilde{\lambda}_{A}=\frac{\alpha_{A} \sqrt{\lambda}-\beta_{A}}{\beta_{A} \sqrt{\lambda}-\alpha_{A}}=\lambda_{A}
$$

Следовательно, $\widetilde{A}=A$. Аналогично $\widetilde{B}=B$.

\section{§ 4. Пространства типа Фрике-Клейна-Тайхмюллера}

1. Пусть $\gamma_{g, n}-$ группа, порожденная образуюшими

$$
v=v_{g, n}=\left\{a_{i}, b_{i}(i=1, \ldots, g), c_{i}(i=g+1, \ldots, n)\right\}
$$

с определяюшим соотношением

$$
\prod_{i=1}^{g}\left[a_{i}, b_{i}\right] \prod_{i=g+1}^{n} c_{i}=1
$$

Положим $c_{i}=\left[a_{i}, b_{i}\right](i=1, \ldots, g)$.

Пусть $n=g+k+m$ и $6 g+3 k+2 m>6$. Обозначим через $\widetilde{T}_{g, k}, m$ множество мономорфизмов $\psi: v \rightarrow \operatorname{Aut}(\Lambda)$ таких, что $\psi(v)$ - последовательньй набор типа $(g, k, m)$. Для $d \in \gamma_{g, n}$ и $D \in \operatorname{Aut}(\Lambda)$ положим

$$
T_{g, k, m}(d, D)=\left\{\psi \in \widetilde{T}_{g, k, m} \mid \psi(d)=D\right\} .
$$

Пусть $k+m>1$. Рассмотрим вложение

$$
\varphi: \gamma_{g, n-1} \rightarrow \gamma_{g, n},
$$

определенное условиями

$$
\begin{gathered}
\varphi\left(a_{i}\right)=a_{i}, \quad \varphi\left(b_{i}\right)=b_{i} \quad(i=1, \ldots, g), \\
\varphi\left(c_{g+1}\right)=c_{n-1} c_{n}, \quad \varphi\left(c_{i}\right)=c_{n}^{-1} c_{n-1}^{-1} c_{i-1} c_{n-1} c_{n} \quad(i=g+2, \ldots, n-1) .
\end{gathered}
$$

Рассмотрим также вложение $\widetilde{\varphi}: \gamma_{0,3} \rightarrow \gamma_{g, n}$, определенное условиями

Гомоморфизм $\psi \in \widetilde{T}_{g, k, m}$ порождает гомоморфизмы

$$
\widetilde{\varphi}\left(c_{1}\right)=c_{n}^{-1} c_{n-1}^{-1}, \quad \widetilde{\varphi}\left(c_{2}\right)=c_{n-1}, \quad \widetilde{\varphi}\left(c_{3}\right)=c_{n} .
$$

$$
\Phi(\psi)=\psi \varphi, \quad \widetilde{\Phi}(\psi)=\psi \widetilde{\varphi} .
$$

Для $\psi^{\prime} \in \Phi\left(\widetilde{T}_{g, k, m}\right)$ обозначим через $\Phi_{\psi^{\prime}}$ ограничение отображения $\widetilde{\Phi}$ на $\Phi^{-1}\left(\psi^{\prime}\right)$.

Положим

$$
\begin{array}{lll}
\left(k^{\prime}, m^{\prime}\right)=(k+1, m-2), & (\widetilde{k}, \widetilde{m})=(1,2) \text { при } m \leqslant 2, \\
\left(k^{\prime}, m^{\prime}\right)=(k, 0), & (\widetilde{k}, \widetilde{m})=(2,1) \text { при } m=1, \\
\left(k^{\prime}, m^{\prime}\right)=(k-1,0), & (\widetilde{k}, \widetilde{m})=(3,0) \text { при } m=0 .
\end{array}
$$

Лемма 4.1. $\Phi\left(\widetilde{T}_{g, k, m}\right)=\widetilde{T}_{g, k^{\prime}, m^{\prime}}$. Для любого $\psi^{\prime} \in \widetilde{T}_{g, k^{\prime}, m^{\prime}}$

$$
\Phi_{\psi^{\prime}}\left(\Phi^{-1}\left(\psi^{\prime}\right)\right)=\widetilde{T}_{0, \widetilde{k}^{\prime}, \widetilde{m}^{\prime}}\left(c_{1}, \psi^{\prime}\left(c_{g+1}^{-1}\right)\right),
$$

причем отображсение $\Phi_{\psi^{\prime}}$ - взаимно однозначно. 
ДокаЗАТЕЛьСтво. Пусть $\psi \in \widetilde{T}_{g, k, m}$ и $\Gamma=\psi\left(\gamma_{g, n}\right)$. Согласно теореме $1.1 \Gamma-$ фуксова группа, а $P=H / \Gamma$ - риманова поверхность рода $g$ с $k$ дырами и $m$ проколами. Согласно лемме 2.1 множество $\psi\left(v_{g, n}\right)$ порождает стандартную систему образующих

$$
\widetilde{v}=\left\{\widetilde{a}_{i}, \widetilde{b}_{i}(i=1, \ldots, g), \widetilde{c}_{i}(i=g+1, \ldots, n)\right\}
$$

групшы $\pi_{1}(P)$. Отсюда (см. рис. 2.2) следует, что набор

$$
\left\{d_{1}, \ldots, d_{r}\right\}=\left\{\widetilde{a}_{1}, \widetilde{b}_{1} \widetilde{a}_{1}^{-1} \widetilde{b}_{1}^{-1}, \ldots, \widetilde{a}_{g}, \widetilde{b}_{g} \widetilde{a}_{g}^{-1} \widetilde{b}_{g}^{-1}, \widetilde{c}_{1}, \ldots, \widetilde{c}_{n}\right\}
$$

таков, что при любом $1<i<g+n$ элементы

$$
d_{1} \cdots d_{i-1}, d_{i}, d_{i+1} \cdots d_{r}
$$

могут быть представлены простыми контурами без попарных пересечений. В этом случае набор

$$
\left\{d_{1}, \ldots, d_{2 g}, d_{r-1} d_{r}, d_{r}^{-1} d_{r-1}^{-1} d_{2 g+1} d_{r-1} d_{r}, \ldots, d_{r}^{-1} d_{r-1}^{-1} d_{r-2} d_{r-1} d_{r}\right\}
$$

также обладает этим свойством. Согласно лемме 2.4 отсюда следует, что $\psi \varphi-$ последовательный набор. Таким образом,

$$
\Phi\left(\widetilde{T}_{g, k, m}\right) \subset \widetilde{T}_{g, k^{\prime}, m^{\prime}}
$$

Величины $\left(k^{\prime}, m^{\prime}\right)$ легко найти из геометрии набора $\widetilde{v}$ (рис. 2.2 ).

Аналогично доказьвается, что $\Phi_{\psi^{\prime}}\left(\Phi^{-1}\left(\widetilde{T}_{g, k^{\prime}, m^{\prime}}\right)\right) \subset \widetilde{T}_{0, \widetilde{k}^{\prime}, \widetilde{m}^{\prime}}\left(c_{1}, \psi^{\prime}\left(c_{g+1}^{-1}\right)\right)$.

Осталось доказать, что

$$
\Phi\left(\widetilde{T}_{g, k, m}\right) \supset \widetilde{T}_{g, k^{\prime}, m^{\prime}},
$$

и для каждого $\psi^{\prime} \in \widetilde{T}_{g, k^{\prime}, m^{\prime}}$ построить отображение

$$
T_{0, \widetilde{k}, \widetilde{m}}\left(c_{1}^{-1}, \psi^{\prime}\left(c_{g+1}\right)\right) \rightarrow \Phi^{-1}\left(\psi^{\prime}\right),
$$

обратное к $\Phi_{\psi^{\prime}}$. Пусть

$$
\widetilde{\psi} \in T_{0, \widetilde{k}, \widetilde{m}}\left(c_{1}^{-1}, \psi^{\prime}\left(c_{g+1}\right)\right) .
$$

Рассмотрим набор сдвигов $\psi\left(v_{g, n}\right)$, заданный условиями

$$
\begin{gathered}
\psi\left(a_{i}\right)=\psi^{\prime}\left(a_{i}\right), \quad \psi\left(b_{i}\right)=\psi^{\prime}\left(b_{i}\right) \quad(i=1, \ldots, g), \\
\psi\left(c_{i}\right)=\psi^{\prime}\left(c_{n-1} c_{n} c_{i-1} c_{n}^{-1} c_{n-1}^{-1}\right) \quad(i=g+2, \ldots, n-2), \\
\psi\left(c_{n-1}\right)=\widetilde{\psi}\left(c_{2}\right), \quad \psi\left(c_{n}\right)=\widetilde{\psi}\left(c_{3}\right) .
\end{gathered}
$$

Из определений следует, что это последовательный набор, порож дающий $\psi \in \widetilde{T}_{g, k, m}$, причем $\Phi(\psi)=\psi^{\prime}$. Таким образом, $\Phi\left(\widetilde{T}_{g, k, m}\right) \supset \widetilde{T}_{g, k^{\prime}, m^{\prime}}$. Нетрудно видеть, что соответствие $\widetilde{\psi} \mapsto \psi$ порождает отображение, обратное к $\Phi_{\psi^{\prime}}$.

2. Пусть $g>0$. Рассмотрим вложение $\varphi^{*}: \gamma_{g-1, n} \rightarrow \gamma_{g, n}$, определяемое условиями $\varphi^{*}\left(a_{i}\right)=a_{i}, \varphi^{*}\left(b_{i}\right)=b_{i}(i=1, \ldots, g-1), \varphi^{*}\left(c_{i}\right)=c_{i}(i=g, \ldots, n)$.

Рассмотрим также вложение $\widetilde{\varphi}^{*}: \gamma_{1,1} \rightarrow \gamma_{g, n}$, определенное условиями

$$
\tilde{\varphi}^{*}\left(a_{1}\right)=a_{g}, \quad \tilde{\varphi}^{*}\left(b_{1}\right)=b_{g}, \quad \tilde{\varphi}^{*}\left(c_{1}\right)=c_{g} .
$$

Гомоморфизм $\psi \in \widetilde{T}_{g, k, m}$ порождает гомоморфизмы

$$
\Omega(\psi)=\psi \varphi^{*}, \quad \widetilde{\Omega}(\psi)=\psi \widetilde{\varphi}^{*} .
$$

Для $\psi^{\prime} \in \Omega\left(\widetilde{T}_{g, k, m}\right)$ обозначим через $\Omega_{\psi^{\prime}}$ ограничение отображения $\widetilde{\Omega}$ на $\Omega^{-1}\left(\psi^{\prime}\right)$. 


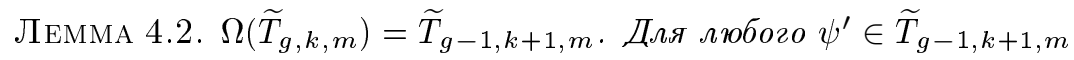

$$
\Omega_{\psi^{\prime}}\left(\Omega^{-1}\left(\psi^{\prime}\right)\right)=\widetilde{T}_{1,1,0}\left(c_{1}, \psi^{\prime}\left(c_{g}\right)\right),
$$

причем $\Omega_{\psi^{\prime}}-$ взаимно однозначно.

ДокАЗАТЕЛЬСтво. Соотношение $\Omega\left(\widetilde{T}_{g, k, m}\right) \subset \widetilde{T}_{g-1, k+1, m}$ сразу следует из наших определений. Осталось доказать, что

$$
\Omega\left(\widetilde{T}_{g, k, m}\right) \supset \widetilde{T}_{g-1, k+1, m},
$$

и для каждого $\psi^{\prime} \in \widetilde{T}_{g-1, k+1, m}$ построить отображение

$$
\widetilde{T}_{1,1,0}\left(c_{1}, \psi^{\prime}\left(c_{g}\right)\right) \rightarrow \Omega^{-1}\left(\psi^{\prime}\right),
$$

обратное к $\Omega_{\psi^{\prime}}$. Пусть

$$
\widetilde{\psi} \in \widetilde{T}_{1,1,0}\left(c_{1}, \psi^{\prime}\left(c_{g}\right)\right) .
$$

Построим набор сдвигов $\psi(g, n)$, заданньй условиями $\psi\left(a_{i}\right)=\psi^{\prime}\left(a_{i}\right), \psi\left(b_{i}\right)=\psi^{\prime}\left(b_{i}\right)$ $(i=1, \ldots, g-1), \psi\left(a_{g}\right)=\widetilde{\psi}\left(a_{1}\right), \psi\left(b_{g}\right)=\widetilde{\psi}\left(b_{g}\right), \psi\left(c_{i}\right)=\psi^{\prime}\left(c_{i}\right)(i=g+1, \ldots, n)$. Из определений следует, что это последовательньй набор, порождаюший $\psi \in \widetilde{T}_{g, k, m}$, причем $\Omega(\psi)=\psi^{\prime}$. Таким образом,

$$
\Omega\left(\widetilde{T}_{g, k, m}\right)=\widetilde{T}_{g-1, k+1, m}
$$

Нетрудно видеть, что соответствие $\widetilde{\psi} \mapsto \psi$ порождает отображение, обратное к $\Omega_{\psi^{\prime}}$.

3. Параметризуем теперь пространство $\widetilde{T}_{g, k, m}$, следуя [23].

Пусть $\psi \in \widetilde{T}_{g, k, m}$ и $n=g+k+m$.

При $m=0$ сопоставим мономорфизму $\psi$ набор чисел

$$
\omega(\psi)=\left(\lambda_{1}, \ldots, \lambda_{n-1}, \xi_{1}, \eta_{1}, \varepsilon_{1}, \ldots, \xi_{g}, \eta_{g}, \varepsilon_{g}, \alpha_{1}, \beta_{1}, \ldots, \alpha_{g+k-1}, \beta_{g+k-1}\right) .
$$

При $m=1$ сопоставим набор

$\omega(\psi)=\left(\lambda_{1}, \ldots, \lambda_{n-1}, \xi_{1}, \eta_{1}, \varepsilon_{1}, \ldots, \xi_{g}, \eta_{g}, \varepsilon_{g}, \alpha_{1}, \beta_{1}, \ldots, \alpha_{g+k-1}, \beta_{g+k-1}, \alpha_{g+k}\right)$.

При $m>1$ - набор

$$
\begin{aligned}
\omega(\psi)=\left(\lambda_{1}, \ldots, \lambda_{n-1}, \xi_{1}, \eta_{1}, \varepsilon_{1}, \ldots, \xi_{g}, \eta_{g},\right. \\
\left.\quad \varepsilon_{g}, \alpha_{1}, \beta_{1}, \ldots, \alpha_{g+k}, \beta_{g+k}, a_{g+k+1}, \ldots, a_{n-2}\right) .
\end{aligned}
$$

Эти числа определяются следующими условиями:

$$
\begin{gathered}
\psi\left(c_{i}\right)(z)=\frac{\left(\lambda_{i} \alpha_{i}-\beta_{i}\right) z+\left(1-\lambda_{i}\right) \alpha_{i} \beta_{i}}{\left(\lambda_{i}-1\right) z+\left(\alpha_{i}-\lambda_{i} \beta_{i}\right)}, \quad \lambda_{i}>1 \text { при } i \leqslant g+k, \\
\psi\left(c_{i}\right)(z)=\frac{\left(1-a_{i} \lambda_{i}\right) z+a_{i}^{2} \lambda_{i}}{-\lambda_{i} z+\left(1+a_{i} \lambda_{i}\right)}, \quad \lambda_{i}>0 \text { при } i>g+k, \\
\psi\left(a_{i}\right)(z)=\frac{\left(\lambda_{i}^{a} \delta_{i}-\eta_{i}\right) z-\left(1-\lambda_{i}^{a}\right) \delta_{i} \eta_{i}}{\left(\lambda_{i}^{a}-1\right) z+\left(\delta_{i}-\lambda_{i}^{a} \eta_{i}\right)}, \\
\psi\left(b_{i}\right)(z)=\frac{\left(\lambda_{i}^{b} \varepsilon_{i}-\xi_{i}\right) z-\left(1-\lambda_{i}^{b}\right) \varepsilon_{i} \xi_{i}}{\left(\lambda_{i}^{b}-1\right) z+\left(\varepsilon_{i}-\lambda_{i}^{b} \xi_{i}\right)} .
\end{gathered}
$$

Таким образом, мы сопоставили мономорфизму $\psi$ набор $\omega(\psi)$ из $6 g+3 k+2 m-3$ чисел. 
Лемма 4.3. Пусть $\psi_{1}, \psi_{2} \in \widetilde{T}_{g, k, m} u \omega\left(\psi_{1}\right)=\omega\left(\psi_{2}\right)$. Тогда $\psi_{1}=\psi_{2}$.

ДоКАЗАТЕЛЬСТво. Согласно определениям величины $\omega(\psi)$ непосредственно определяют $\psi\left(c_{i}\right)$. Уравнения $(3.8)$ и $(3.9)$ позволяют однозначно найти $\psi\left(a_{i}\right), \psi\left(b_{i}\right)$ через $\psi\left(c_{i}\right)$ и $\omega(\psi)$.

Таким образом, $\omega(\psi)$ дает глобальную систему координат на $\widetilde{T}_{g, k, m}$.

Группа $\operatorname{Aut}(\Lambda)$ действует на $\widetilde{T}_{g, k, m}$ сопряжением по правилу $\psi \mapsto h^{*} \psi$, где $\psi \in \widetilde{T}_{g, k, m}, h \in \operatorname{Aut}(\Lambda), h^{*} \psi(d)=h \psi(d) h^{-1}$. Положим

$$
T_{g, k, m}=\widetilde{T}_{g, k, m} / \operatorname{Aut}(\Lambda) .
$$

Если $\omega(\psi)=\left(\lambda_{1}, \ldots, \lambda_{n-1}, \xi_{1}, \eta_{1}, \ldots, a_{n-2}\right)$, то

$$
\omega\left(h^{*} \psi\right)=\left(\lambda_{1}, \ldots, \lambda_{n-1}, h \xi_{1}, h \eta_{1}, \ldots, h a_{n-2}\right) .
$$

Это позволяет отождествить

$$
\begin{aligned}
& T_{0,0, m}=\left\{\psi \in \widetilde{T}_{0,0, m} \mid \psi\left(c_{1} c_{2}\right)(0)=0, \psi\left(c_{1} c_{2}\right)(\infty)=\infty, a_{1}=-1\right\}, \\
& T_{0,1, m}=\left\{\psi \in \widetilde{T}_{0,1, m} \mid \alpha_{1}=\infty, \beta_{1}=0, a_{2}=1\right\} \\
& T_{1,1, m}=\left\{\psi \in \widetilde{T}_{1,1, m} \mid \alpha_{1}=\infty, \beta_{1}=0, \xi_{1}=1\right\}
\end{aligned}
$$

и в остальных случаях

$$
T_{g, k, m}=\left\{\psi \in \widetilde{T}_{g, k, m} \mid \alpha_{1}=\infty, \beta_{1}=0, \alpha_{2}=1\right\} .
$$

Опишем теперь отображение

$$
\Psi: T_{g, k, m} \rightarrow \mathbb{R}^{6 g+3 k+2 m-6} .
$$

При $g=k=0$ положим

$$
\Psi(\psi)=\left\{\lambda_{1}, \lambda_{3}, \lambda_{4}, \ldots, \lambda_{m-1}, a_{3}, \ldots, a_{m-2}\right\} \subset \mathbb{R}^{2 m-6} .
$$

При $g=0, k=1$ положим

$$
\Psi(\psi)=\left\{\lambda_{1}, \lambda_{2}, \ldots, \lambda_{m}, a_{3}, \ldots, a_{m-1}\right\} \subset \mathbb{R}^{2 m-3} .
$$

При $g=k=1$ положим

$$
\Psi(\psi)=\left\{\lambda_{1}, \ldots, \lambda_{m+1}, \eta_{1}, \varepsilon_{1}, \alpha_{2}, \beta_{2}, a_{3}, a_{4}, \ldots, a_{m}\right\} \subset \mathbb{R}^{2 m+3} .
$$

В остальных случаях:

$$
\begin{aligned}
& \text { при } m=0 \text { положим } \\
& \Psi(\psi)=\left\{\lambda_{1}, \ldots, \lambda_{g+k-1}, \xi_{1}, \eta_{1}, \varepsilon_{1}, \ldots, \xi_{g}, \eta_{g}, \varepsilon_{g}, \beta_{2},\right. \\
&\left.\alpha_{3}, \beta_{3}, \ldots, \alpha_{g+k-1}, \beta_{g+k-1}\right\} \subset \mathbb{R}^{6 g+3 k-6}, \\
& \text { при } m=1 \\
& \Psi(\psi)=\left\{\lambda_{1}, \ldots, \lambda_{g+k}, \xi_{1}, \eta_{1}, \varepsilon_{1}, \ldots, \xi_{g}, \eta_{g}, \varepsilon_{g}, \beta_{2},\right. \\
&\left.\alpha_{3}, \beta_{3}, \ldots, \alpha_{g+k-1}, \beta_{g+k-1}, \alpha_{g+k}\right\} \subset \mathbb{R}^{6 g+3 k-4},
\end{aligned}
$$

а в остальных случаях

$$
\begin{aligned}
\Psi(\psi)=\left\{\lambda_{1}, \ldots, \lambda_{g+k+m-1}, \xi_{1}, \eta_{1}, \varepsilon_{1}, \ldots, \xi_{g}, \eta_{g}, \varepsilon_{g}, \beta_{2},\right. \\
\left.\alpha_{3}, \beta_{3}, \ldots, \alpha_{g+k}, \beta_{g+k}, a_{g+k+1}, \ldots, a_{g+k+m-2}\right\} \subset \mathbb{R}^{6 g+3 k+2 m-6} .
\end{aligned}
$$

Положим

$$
\widehat{T}_{g, k, m}=\Psi\left(T_{g, k, m}\right) .
$$


Tеорема 4.1 [23]. Отображение $\Psi: T_{g, k, m} \rightarrow \widehat{T}_{g, k, m}$ является диффеоморфизмом и $\widehat{T}_{g, k, m}$ является открытой областью в $\mathbb{R}^{6 g+3 k+2 m-6}$, гомеоморфной $\mathbb{R}^{6 g+3 k+2 m-6}$.

ДокАЗАТЕЛЬСТво. Будем доказывать индукцией по $2 g+k+m$. Для $T_{1,1,0}, T_{0,3,0}$, $T_{0,2,1}, T_{0,1,2}$ утверждение теоремы следует из лемм 3.2-3.4. Докажем утверждение для $T_{g, k, m}$ в предположении, что оно верно для всех $T_{g^{\prime}, k^{\prime}, m^{\prime}}$, где $2 g^{\prime}+k^{\prime}+m^{\prime}<$ $2 g+k+m$. Пусть $g>0$. Тогда согласно лемме $4.2 \widetilde{T}_{g, k, m}=\{\psi\}$ представляется в виде расслоения над $\widetilde{T}_{g-1, k+1, m}$ со слоем, гомеоморфным $\widetilde{T}_{1,1,0}\left(c_{1}^{-1}, \psi\left(c_{g}\right)\right)$. Таким образом, $T_{g, k, m}$ представляется в виде расслоения над $T_{g-1, k+1, m}$ со слоем, гомеоморфным

$$
\left\{\psi \in \widetilde{T}_{1,1,0} \mid \psi\left(c_{1}\right)(z)=\lambda_{g} z\right\} .
$$

Согласно лемме 3.4 последнее множество гомеоморфно $\mathbb{R}^{3}$. По предположению индукции отсюда следует, что

$$
T_{g, k, m} \cong \mathbb{R}^{6 g+3 k+2 m-6} \text {. }
$$

Пусть $g=0$. Тогда согласно лемме $4.1 \widetilde{T}_{0, k, m}=\{\psi\}$ представляется в виде расслоения над $\widetilde{T}_{0, k^{\prime}, m^{\prime}}$ со слоем $\widetilde{T}_{0, \widetilde{k}, \widetilde{m}}\left(c_{1}^{-1}, \psi\left(c_{1}\right)\right)$. Таким образом, $T_{0, k, m}$ представляется в виде расслоения над $T_{0, k^{\prime}, m^{\prime}}$ со слоем, гомеоморфным $\widetilde{T}_{0,3,0}\left(c_{1}^{-1}, \psi\left(c_{1}\right)\right)$, $\widetilde{T}_{0,2,1}\left(c_{1}^{-1}, \psi\left(c_{1}\right)\right)$ или $\widetilde{T}_{0,1,2}\left(c_{1}^{-1}, \psi\left(c_{1}\right)\right)$. Согласно леммам $3.2,3.3$ последние множества гомеоморфны соответственно $\mathbb{R}^{3}, \mathbb{R}^{2}$ и $\mathbb{R}^{1}$. По предположению индукции отсюда следует, что

$$
T_{0, k, m} \cong \mathbb{R}^{3 k+2 m-6} .
$$

ЗАмечАниЕ. Неравенства, ограничиваюшие $\widehat{T}_{g, k, m}$ в $\mathbb{R}^{6 g+3 k+2 m-6}$, становятся линейньми и квадратичньми, если перейти от параметров $\lambda_{i}$ к $\mu_{i}=\sqrt{\lambda_{i}}$.

\section{§5. Модули римановых поверхностей}

1. Пусть $\psi \in \widetilde{T}=\widetilde{T}_{g, k, m}$. По определению это отображение $\psi: v \rightarrow \operatorname{Aut}(\Lambda)$ (где $\left.v=v_{g, g+k+m}\right)$, наделенное некоторьми специальными свойствами. Положим

$$
\widetilde{\operatorname{Mod}}^{\psi}=\widetilde{\operatorname{Mod}}_{g, k, m}^{\psi}=\{\alpha \in \operatorname{Aut}(\gamma) \mid \psi \alpha \in \widetilde{T}\},
$$

где $\gamma$ - группа, порожденная $v$.

Отображению $\psi \in \widetilde{T}$ отвечает фуксова группа $\Gamma^{\psi}=\psi(\gamma)$ и риманова поверхность $P^{\psi}=\Lambda / \Gamma^{\psi}$. Рассмотрим группу $G_{p}$ автогомеоморфизмов $P^{\psi}$, сохраняюших точку $p \in P^{\psi}$, ориентацию $P^{\psi}$ и переводяшую дыры в дыры и проколы в проколы. Рассмотрим подгруппу $G_{p}^{\prime} \subset G_{p}$, состояшую из гомеоморфизмов, получаюшихся из тождественного гомотопией, сохраняющей $p$. Положим $\widetilde{\operatorname{Mod}}_{p}^{\psi}=G_{p} / G_{p}^{\prime}$.

Лемма 5.1. Группы $\widetilde{\operatorname{Mod}}^{\psi} u \widetilde{\operatorname{Mod}}_{p}^{\psi}$ изоморфны. 
ДокаЗАтельСтво. Рассмотрим униформизацию $\Phi: \Lambda \rightarrow P^{\psi}$. Пусть $q \in \Phi^{-1}(p)$. Сопоставим элементу $\gamma \in \Gamma^{\psi}$ элемент $\Phi\left(\ell_{\gamma}\right) \in \pi_{1}\left(P^{\psi}, p\right)$, где $\ell_{\gamma} \subset \Lambda$ - отрезок, соединяюший $q$ и $\gamma(q)$. Соответствие $\gamma \mapsto \Phi\left(\ell_{\gamma}\right)$ порождает изоморфизм $\Phi_{q}: \Gamma^{\psi} \rightarrow$ $\pi_{1}\left(P^{\psi}, p\right)$. Пусть теперь $\alpha \in \widetilde{\operatorname{Mod}} \psi$. Согласно лемме 2.1 последовательному набору $\psi(v)$ отвечает стандартная система образующих групшы $\pi_{1}\left(P^{\psi}, p\right)$. Представим ее контурами, непересекающимися вне $p$. Их прообраз высекает на $\Lambda$ фундаментальную область $M$ с вершиной $q$. Аналогичную область $M^{\alpha}$ порождает последовательный набор $\psi \alpha(v)$. Таким образом, сушествует гомеоморфизм $A: \Lambda \rightarrow \Lambda$ такой, что $A(M)=M^{\alpha}$ и $A \Gamma^{\psi} A^{-1}=\Gamma^{\psi}$. На поверхности $P^{\psi}$ он порождает автогомеоморфизм $\varphi_{\alpha} \in \widehat{\operatorname{Mod}}_{p}^{\psi}$.

Соответствие $\alpha \mapsto \varphi_{\alpha}$ порождает гомоморфизм $\varphi_{q}: \widetilde{\operatorname{Mod}}^{\psi} \rightarrow \widetilde{\operatorname{Mod}}_{p}^{\psi}$. Нетрудно видеть, что это мономорфизм. Эпиморфизм следует из теоремы 2.1 .

Лемма 5.2. Пусть $\psi, \psi^{\prime} \in \widetilde{T}$. Тогда $\widetilde{\operatorname{Mod}} \psi=\widetilde{\operatorname{Mod}} \psi^{\prime}$.

ДокаЗАтельство. Пусть $\varphi_{q}: \widetilde{\operatorname{Mod}}^{\psi} \rightarrow \widetilde{\operatorname{Mod}}_{p}^{\psi}$ и $\varphi_{q}^{\prime}: \widetilde{\operatorname{Mod}}^{\psi^{\prime}} \rightarrow \widetilde{\operatorname{Mod}}_{p^{\prime}}^{\psi^{\prime}}-$ изоморфизмы, построенные при доказательстве леммы 5.1. Рассмотрим стандартные системы образуюших $v$ и $v^{\prime}$ групп $\pi_{1}\left(P^{\psi}, p\right)$ и $\pi_{1}\left(P^{\psi^{\prime}}, p^{\prime}\right)$. Рассмотрим гомеоморфизм $\beta: P \rightarrow P^{\prime}$, переводящий $v$ в $v^{\prime}$. Он индуцирует изоморфизм $\widetilde{\beta}: \widetilde{\operatorname{Mod}_{p}} \rightarrow \widetilde{\operatorname{Mod}_{p^{\prime}}} \psi^{\prime}$. Согласно нашим определениям и лемме $5.1 \varphi=\varphi_{q}^{-1} \widetilde{\beta}^{-1} \varphi_{q^{\prime}}^{\prime}$ порождает изоморфизм $\varphi: \widetilde{\operatorname{Mod}} \psi^{\prime} \rightarrow \widetilde{\operatorname{Mod}} \psi$.

Следуя лемме 5.2, мы будем писать $\widetilde{\operatorname{Mod}}$ вместо $\widetilde{\operatorname{Mod}} \psi$.

Всякий $h \subset \gamma$ порождает принадлежаший $\widehat{\operatorname{Mod}}$ автоморфизм $d \mapsto h d h^{-1}$ групшы $\gamma$. Пусть $\widetilde{\mathrm{Mod}} \subset \widetilde{\mathrm{Mod}}-$ подгруппа всех таких автоморфизмов и $\mathrm{Mod}=\widetilde{\operatorname{Mod}} / \widehat{\mathrm{IMod}}$.

Аналогично всякий $h \in \pi\left(P^{\psi}, p\right)$ порождает принадлежаший $\widetilde{\operatorname{Mod}}_{p}^{\psi}$ автоморфизм $d \mapsto h d h^{-1}$ группы $\pi_{1}\left(P^{\psi}, p\right)$. Пусть $\widetilde{\operatorname{Mod}}_{p}^{\psi} \subset \widetilde{\operatorname{Mod}}_{p}^{\psi}-$ подгруппа всех таких автоморфизмов и $\operatorname{Mod}_{p}^{\psi}=\widetilde{\operatorname{Mod}_{p}^{\psi}} / \widetilde{\operatorname{Mod}_{p}}$. Движение точки $p$ задает естественные изоморфизмы между группами $\operatorname{Mod}_{p}^{\psi}$, отвечающими разным $p$. Таким образом, каждая из них естественно изоморфна группе $\operatorname{Mod}_{*}^{\psi}=\widetilde{\operatorname{Mod}_{*}} \psi / \widetilde{\operatorname{Mod}_{*}} \psi$, где $\widetilde{\operatorname{Mod}_{*}} \psi-$ группа coxраняюших ориентацию автогомеоморфизмов $P^{\psi}$, переводяших дыры в дыры, а проколы в проколы, а $\widetilde{\mathrm{IMod}_{*}} \psi \subset \widetilde{\operatorname{Mod}_{*}} \psi$ - подгруппа гомотопных тождественному автогомеоморфизмов. Переводящие дыры в дыры и проколы в проколы гомеоморфизмы $P^{\psi} \rightarrow P^{\psi^{\prime}}$ порождают естественные изоморфизмы $\widetilde{\operatorname{Mod}}_{*}^{\psi} \rightarrow{\widetilde{\operatorname{Mod}_{*}}}^{\prime}$. Поэтому в дальнейшем мы будем опускать значки $\psi$ и считать $\widetilde{\operatorname{Mod}}_{*}=\widetilde{\operatorname{Mod}}_{*}^{\psi}, \operatorname{Mod}_{*}=\operatorname{Mod}_{*}^{\psi}$. Из леммы 5.1 следует

СЛЕДСТВИЕ 5.1. Групnа $\operatorname{Mod}=\operatorname{Mod}_{g, k}, m$ естественно изоморфна группе $\operatorname{Mod}_{*}=\operatorname{Mod}_{*}^{g, k, m}$ гомотопических классов, сохраняющих ориентацию автогомеоморфизмов поверхности типа $(g, k, m)$.

2. Перейдем теперь к описанию пространства модулей (т.е. классов биголоморфной эквивалентности) римановых поверхностей. 
Teоpema 5.1. Группа $\operatorname{Mod}_{g, k, m}$ естественно действует на $T_{g, k, m}$ диффеоморфизмами. Это действие дискретно. Фактор-множество $T_{g, k, m} / \operatorname{Mod}_{g, k, m}$ естественно отохсествляется с пространством модулей $M_{g, k, m}$ риманових поверхностей типа $(g, k, m)$.

ДокАЗАтЕльство. Согласно лемме 5.2 группа $\widetilde{\operatorname{Mod}}=\widetilde{\operatorname{Mod}}_{g, k}, m$ естественно действует на множестве $\widetilde{T}=\widetilde{T}_{g, k, m}$. На этом же множестве действует группа $\operatorname{Aut}(\Lambda)$. Нетрудно видеть, что $\widetilde{\operatorname{Mod}} \cap \operatorname{Aut}(\Lambda)=\widetilde{\mathrm{IMod}}$. Таким образом, группа Mod естественно действует на множестве $T=T_{g, k, m}$. Введенные в $\S 4$ координаты на $T_{g, k, m}=\{\psi\}$ явньми аналитическими формулами описьвают последовательный набор $\psi(v)$ как подмножество $(P S L(2, \mathbb{R}))^{2 g+k+m-1}$. Поэтому аналитичность действия Моd на $T$ следует из аналитичности операции в группе $P S L(2, \mathbb{R})$.

Рассмотрим отображение $\widetilde{\Phi}: \widetilde{T} \rightarrow M=M_{g, k, m}$, где $\widetilde{\Phi}(\psi)=P^{\psi}$. Очевидно, что $\widetilde{\Phi}$ принимает одинаковые значения на орбитах действия групп $\operatorname{Aut}(\Lambda)$ и $\widetilde{\operatorname{Mod}}$. Таким образом, $\widetilde{\Phi}$ индуцирует отображение $\Phi: T / \operatorname{Mod} \rightarrow M$. Согласно теореме униформизации всякая $P \in M$ имеет вид $\Lambda / \Gamma$, где $\Gamma$ - фуксова группа. Таким образом, согласно теореме $2.1 \Phi(T)=\widetilde{\Phi}(\widetilde{T})=M$.

Докажем теперь, что $\Phi(x) \neq \Phi\left(x^{\prime}\right)$ при $x \neq x^{\prime}$. Действительно, пусть $\psi \in x, \psi^{\prime} \in x^{\prime}$ и $\widetilde{\Phi}(\psi)=\widetilde{\Phi}\left(\psi^{\prime}\right)$. По определению это означает, что римановы поверхности $P^{\psi}$ и $P^{\psi^{\prime}}$ биголоморфно эквивалентны. Следовательно, $\psi$ и $\psi^{\prime}$ можно выбрать таким образом, чтобы униформизирующие группы $\Gamma^{\psi}$ и $\Gamma^{\psi^{\prime}}$ совпадали. Таким образом, сушествует $\alpha \in \widetilde{\operatorname{Mod}} \psi$ такой, что $\psi^{\prime}=\psi \alpha$. Следовательно, согласно лемме $5.2 \alpha \in \widetilde{\operatorname{Mod}}$ и, значит, $x=x^{\prime}$.

Сопоставим каждому $\psi \in \widetilde{T}$ набор длин геодезических на поверхности $P^{\psi}$. Длина геодезической, отвечающей $C \in \Gamma^{\psi}$, равна $\ln \frac{1}{4}\left(\lambda+\frac{1}{\lambda}+\sqrt{\lambda+\frac{1}{\lambda}}-2\right)$, где $\lambda$ - параметр сдвига $C$ [22]. Таким образом, дискретность действия Mod относительно наших координат следует из дискретности множества длин геодезических [22].

Согласно теореме 5.1 введенная в $§ 4$ аналитическая структура на $T$ порождает аналитическую структуру на пространстве модулей $M$. Именно эту структуру мы будем иметь в виду, исследуя топологию $M$. Теоремы 4.1 и 5.1 дают

СлЕДСТВИЕ 5.2 [18]. Пространство модулей римановых поверхностей типа $(g, k, m)$ диффеоморфно $\mathbb{R}^{6 g+3 k+2 m-6} / \operatorname{Mod}_{g, k, m}$, где $\operatorname{Mod}_{g, k, m}-$ дискретная групna.

\section{§. Пространство голоморфных морфизмов римановых поверхностей}

1. Голоморфным морфизмом степени $d$ мы назьваем тройку $(\widetilde{P}, f, P)$, где $\widetilde{P}$ и $P$ - римановы поверхности, а $f: \widetilde{P} \rightarrow P$ - голоморфное отображение степени $d$ такое, что $f(\widetilde{P})=P$. Морфизмы $\left(\widetilde{P}_{1}, f_{1}, P_{1}\right)$ и $\left(\widetilde{P}_{2}, f_{2}, P_{2}\right)$ считаются совпадаюшими, если сушествуют биголоморфные отображения $\widetilde{\varphi}: \widetilde{P}_{1} \rightarrow \widetilde{P}_{2}$ и $\widetilde{\varphi}: P_{1} \rightarrow P_{2}$ такие, что $\varphi f_{1}=$ $f_{2} \widetilde{\varphi}$.

Морфизмы $\left(\widetilde{P}_{1}, f_{1}, P_{1}\right)$ и $\left(\widetilde{P}_{2}, f_{2}, P_{2}\right)$ назьваются топологически эквивалентными, если существуют гомеоморфизмы $\widetilde{\varphi}: \widetilde{P}_{1} \rightarrow \widetilde{P}_{2}$ и $\varphi: P_{1} \rightarrow P_{2}$ такие, что $\varphi f_{1}=f_{2} \widetilde{\varphi}$. 
Класс топологической эквивалентности называется топологическим типом. Зафиксируем топологический тип $t$ и рассмотрим множество $H^{t}$ всех морфизмов этого топологического типа.

Пример. Если $P$ - сфера Римана, то $(\widetilde{P}, f, P)$ - это мероморфная функция на $\widetilde{P}$. Если $\widetilde{P}$ - тоже сфера Римана, то $f$ - рациональная функция. Если, кроме того, $f^{-1}(\infty)=\infty$, то $f-$ полином. Критическим значением полинома назьвается число $f(x)$, где $d f(x)=0$. Если все эти числа различны, то говорят, что это полином общего положения. Все такие полиномы топологически эквивалентны. Однако в общем случае характер критических значений не определяет топологический тип полинома [31]. $\mathrm{K}$ множествам вида $H^{t}$ относятся также лорановские полиномы обшего положения [32] и пространства мероморфных функций с фиксированным типом полюсов [33]-[35], возникающих в топологической теории поля [10].

Топологический тип однозначно определяет топологический тип $(g, k, m)$ поверхности $P$, накрываемой морфизмом $(\widetilde{P}, f, P) \in H^{t}$. Кроме того, $t$ определяет число $b$ критических значений

$$
B=B(\widetilde{P}, f, P)=\{f(\widetilde{p}) \in P \mid \widetilde{p} \in \widetilde{P}, d f(\widetilde{p})=0\}
$$

Далее мы считаем, что $b<\infty$. Тогда соответствие $(\widetilde{P}, f, P) \mapsto P \backslash B$ задает отображение

$$
\Psi: H^{t} \rightarrow M_{g, k, m+b}
$$

В $\S 5$ мы ввели топологию в пространстве $M_{g, k}, m+b$. Введем теперь топологию в пространстве $H^{t}$, потребовав, чтобы функция $\Psi$ была непрерывна.

2. Пусть теперь $\psi \in \widetilde{T}_{g, k, m+b}$ и $\widetilde{\gamma}-$ подгруппа индекса $d$ групшы $\gamma=\gamma_{g, n}$, где $n=g+k+m+b$. Положим

$$
\Gamma^{\psi}=\psi(\gamma), \quad \Gamma_{\widetilde{\gamma}}^{\psi}=\psi(\widetilde{\gamma}), \quad P^{\psi}=\Lambda / \Gamma^{\psi} \text { и } P_{\widetilde{\gamma}}^{\psi}=\Lambda \backslash \Gamma_{\widetilde{\gamma}}^{\psi}
$$

Тогда естественное вложение $\Gamma_{\widetilde{\gamma}}^{\psi} \subset \Gamma^{\psi}$ индуцирует морфизм

$$
f_{\widetilde{\gamma}}^{\psi}: P_{\widetilde{\gamma}}^{\psi} \rightarrow P^{\psi}
$$

степени $d$. Заклеим теперь проколы на $P^{\psi}$ и $P_{\widetilde{\gamma}}^{\psi}$, порожденные параболическими сдвигами $\psi\left(c_{g+k+m+1}\right), \ldots, \psi\left(c_{n}\right)$. В итоге получим морфизм

$$
\left(P_{\widetilde{\gamma}}^{\psi}, f_{\widetilde{\gamma}}^{\psi}, P^{\psi}\right)^{b}
$$

ЛЕмма 6.1. Всякий голоморфный морфизм имеет вид

$$
\left(P_{\widetilde{\gamma}}^{\psi}, f_{\widetilde{\gamma}}^{\psi}, P^{\psi}\right)^{b}
$$

əде $\psi \in \widetilde{T}_{g, k, m+b} u \widetilde{\gamma} \subset \gamma_{g, n}$. 
ДокАЗАТЕЛЬСТвО. Пусть $(\widetilde{P}, f, P)$ - произвольный голоморфный морфизм. Положим $P^{*}=P \backslash B(\widetilde{P}, f, P) \in M_{g, k, m+b}$ и $\widetilde{P}^{*}=f^{-1}\left(P^{*}\right)$. Согласно теореме 5.1 сушествует $\psi \in \widetilde{T}_{g, k, m+b}$ такой, что $P^{\psi}=P^{*}$. Согласно теореме 2.1 можно считать, что $\psi\left(c_{g+k+m+1}\right), \ldots, \psi\left(c_{n}\right)$ отвечают проколам из множества $B=B(\widetilde{P}, f, P) \subset P$.

Группа $\Gamma^{\psi}=\psi(\gamma)$ является группой монодромий универсального накрытия $\Phi: \Lambda \rightarrow P^{*}$. Ввиду универсальности накрытие $\Phi$ может быть "пропушено" через произвольное накрытие поверхности $P^{*}$, и, в частности, существует универсальное накрытие $\widetilde{\Phi}: \Lambda \rightarrow \widetilde{P}^{*}$ такое, что $\Phi=f \widetilde{\Phi}$. Пусть $\widetilde{\Gamma}^{\psi} \subset \Gamma^{\psi}-$ группа монодромий этого накрытия. Положим $\widetilde{\gamma}=\psi^{-1}\left(\widetilde{\Gamma}^{\psi}\right)$. Тогда

$$
\left(P_{\widetilde{\gamma}}^{\psi}, f_{\widetilde{\gamma}}^{\psi}, P^{\psi}\right)=\left(\widetilde{P}^{*}, f^{*}, P^{*}\right)
$$

где $f^{*}=\left.f\right|_{\widetilde{P}^{*}}$. Заклеивание проколов $B$ порождает

$$
\left(P_{\widetilde{\gamma}}^{*}, f_{\widetilde{\gamma}}^{\psi}, P^{\psi}\right)^{b}=(\widetilde{P}, f, P) .
$$

Лемма 6.2. Пусть $\psi, \psi^{\prime} \in \widetilde{T}_{g, k, m+b} u \widetilde{\gamma} \subset \gamma_{g, n}-$ подгруппа. Тогда $\left(P_{\widetilde{\gamma}}^{\psi}, f_{\widetilde{\gamma}}^{\psi}, P^{\psi}\right)^{b}$ и $\left(P_{\widetilde{\gamma}}^{\psi^{\prime}}, f_{\widetilde{\gamma}}^{\psi^{\prime}}, P^{\psi^{\prime}}\right)^{b}$ имеют одинаковый топологический тип. Если, кроме того, существует $A \in \operatorname{Aut}(\Lambda)$ такой, что $A \psi(w) A^{-1}=\psi^{\prime}(w)$ для всех $w \in v_{g, n}$, mо

$$
\left(P_{\widetilde{\gamma}}^{\psi}, f_{\widetilde{\gamma}}^{\psi}, P^{\psi}\right)^{b}=\left(P_{\widetilde{\gamma}}^{\psi^{\prime}}, f_{\widetilde{\gamma}}^{\psi^{\prime}}, P^{\psi^{\prime}}\right)^{b} .
$$

ДокАЗАТЕЛЬСТво. Положим $v=v_{g, g+k+m+b}$. Согласно лемме 2.1 наборы $\psi(v)$ и $\psi^{\prime}(v)$ при естественных накрытиях $\Phi: \Lambda \rightarrow P^{\psi}, \Phi^{\prime}: \Lambda \rightarrow P^{\psi^{\prime}}$ переходят в стандартные системы образуюших групा $\pi_{1}\left(P^{\psi}, p\right)$ и $\pi_{1}\left(P^{\psi^{\prime}}, p^{\prime}\right)$. Следовательно, существует гомеоморфизм $\varphi: P^{\psi} \rightarrow P^{\psi^{\prime}}$, переводящий одну стандартную систему в другую. Проекции $\Phi_{q}$ и $\Phi_{q}^{\prime}$ "поднимают" его до гомеоморфизма $A: \Lambda \rightarrow \Lambda$ такого, что $A \psi(w) A^{-1}=\psi^{\prime}(w)$ для любого $w \in v$. Отсюда следует, что $A \psi(\widetilde{\gamma}) A^{-1}=\psi^{\prime}(\widetilde{\gamma})$. Следовательно, $A$ индуцирует гомеоморфизм $\widetilde{\varphi}: \widetilde{P}^{\psi} \rightarrow \widetilde{P}^{\psi^{\prime}}$ такой, что $\varphi f=f^{\prime} \widetilde{\varphi}$. Если, кроме того, $A \in \operatorname{Aut}(\Lambda)$, то $\varphi$ и $\widetilde{\varphi}$ - голоморфные отображения и, следовательно,

$$
\left(P_{\widetilde{\gamma}}^{\psi}, f_{\widetilde{\gamma}}^{\psi}, P^{\psi}\right)^{b}=\left(P_{\widetilde{\gamma}}^{\psi^{\prime}}, f_{\widetilde{\gamma}}^{\psi^{\prime}}, P^{\psi^{\prime}}\right)^{b} .
$$

Выделим в группе $\gamma=\gamma_{g, n}$, порожденной

$$
v_{g, n}=\left\{a_{i}, b_{i}(i=1, \ldots, g), c_{i}(i=g+1, \ldots, n)\right\},
$$

подмножество $c$ элементов вида $h c_{i} h^{-1}$, где $i>g+k+m, h \in \gamma$.

Положим

$$
\widetilde{\operatorname{Mod}}_{g, k, m+b}^{b}=\left\{\alpha \in \widetilde{\operatorname{Mod}}_{g, k, m+b} \mid \alpha(c)=c\right\} .
$$

Лемма 6.3. Морфизмы $\left(P_{\widetilde{\gamma}}^{\psi}, f_{\widetilde{\gamma}}^{\psi}, P^{\psi}\right)^{b} u\left(P_{\gamma^{\prime}}^{\psi}, f_{\gamma^{\prime}}^{\psi}, P^{\psi}\right)^{b}$ имеют одинаковый топологический тип, если и только если $\gamma^{\prime}=\alpha(\widetilde{\gamma})$, где $\alpha \in \widetilde{\operatorname{Mod}}_{g, k, m+b}^{b}$. 
ДокаЗАТельСтво. Пусть $\widetilde{\varphi}: P_{\widetilde{\gamma}}^{\psi} \rightarrow P_{\gamma^{\prime}}^{\psi}$ и $\varphi: P^{\psi} \rightarrow P^{\psi}$ - гомеоморфизмы, порождаюшие топологическую эквивалентность $\left(P_{\widetilde{\gamma}}^{\psi}, f_{\widetilde{\gamma}}^{\psi}, P^{\psi}\right)^{b}$ и $\left(P_{\gamma^{\prime}}^{\psi}, f_{\gamma^{\prime}}^{\psi}, P^{\psi^{\prime}}\right)^{b}$. Естественные накрытия $\Phi: \Lambda \rightarrow P_{\widetilde{\gamma}}^{\psi} \rightarrow P^{\psi}$ и $\Phi^{\prime}: \Lambda \rightarrow P_{\gamma^{\prime}}^{\psi} \rightarrow P^{\psi}$ поднимают их до гомеоморфизма $A: \Lambda \rightarrow \Lambda$ такого, что $\psi(\gamma)=A \psi(\gamma) A^{-1}$ и $\psi\left(\gamma^{\prime}\right)=A \psi(\widetilde{\gamma}) A^{-1}$. Обозначим через $A^{*}: \psi(\gamma) \rightarrow \psi(\gamma)$ гомоморфизм $A^{*}(h)=A h A^{-1}$ и положим $\alpha=\psi^{-1} A^{*} \psi: \gamma \rightarrow \gamma$. Тогда $\alpha \in \widetilde{\operatorname{Mod}}_{g, k, m+b}^{b}$ и $\alpha(\widetilde{\gamma})=\gamma^{\prime}$.

Пусть теперь $\gamma^{\prime}=\alpha(\widetilde{\gamma})$, где $\alpha \in \widetilde{\operatorname{Mod}}_{g, k, m+b}^{b}$. Положим $\psi^{\prime}=\psi \alpha \in \widetilde{T}_{g, k, m+b}$. Тогда

$$
\left(P_{\widetilde{\gamma}}^{\psi^{\prime}}, f_{\widetilde{\gamma}}^{\psi^{\prime}}, P^{\psi^{\prime}}\right)^{b}=\left(P_{\gamma^{\prime}}^{\psi}, f_{\gamma^{\prime}}^{\psi}, P^{\psi}\right)^{b},
$$

причем согласно лемме $6.2\left(P_{\widetilde{\gamma}}^{\psi^{\prime}}, f_{\widetilde{\gamma}}^{\psi^{\prime}}, P^{\psi^{\prime}}\right)^{b}$ и $\left(P_{\widetilde{\gamma}}^{\psi}, f_{\widetilde{\gamma}}^{\psi}, P^{\psi}\right)^{b}$ имеют одинаковый топологический тип.

Лемма 6.4. Пусть $\left(P_{\widetilde{\gamma}}^{\psi}, f_{\tilde{\gamma}}^{\psi}, P^{\psi}\right) \in H^{t} . \quad$ Тогда $H^{t}=\left\{\left(P_{\widetilde{\gamma}}^{\psi^{\prime}}, f_{\widetilde{\gamma}}^{\psi^{\prime}}, P^{\psi^{\prime}}\right)^{b} \mid \psi^{\prime} \in\right.$ $\left.\widetilde{T}_{g, k, m+b}\right\}$.

ДоКАЗАТЕЛЬСТво. Согласно лемме 6.2

$$
\left\{\left(P_{\widetilde{\gamma}}^{\psi^{\prime}}, f_{\widetilde{\gamma}}^{\psi^{\prime}}, P^{\psi^{\prime}}\right)^{b} \mid \psi^{\prime} \in \widetilde{T}_{g, k, m+b}\right\} \subset H^{t} .
$$

Докажем обратное включение. Пусть $(\widetilde{P}, f, P) \in H^{t}$. Согласно лемме 6.1

$$
(\widetilde{P}, f, P)=\left(P_{\gamma^{\prime}}^{\psi^{\prime}}, f_{\gamma^{\prime}}^{\psi^{\prime}}, P^{\psi^{\prime}}\right)^{b},
$$

где согласно лемме $6.3 \gamma^{\prime}=\alpha(\widetilde{\gamma})$ для $\alpha \in{\widetilde{\operatorname{Mod}_{g, k}}}^{b}, m+b$. Положим $\psi^{*}=\psi^{\prime} \alpha$. Тогда

$$
\left(P_{\gamma^{\prime}}^{\psi^{\prime}}, f_{\gamma^{\prime}}^{\psi^{\prime}}, P^{\psi^{\prime}}\right)^{b}=\left(P_{\widetilde{\gamma}}^{\psi^{*}}, f_{\widetilde{\gamma}}^{\psi^{*}}, P^{\psi^{*}}\right)^{b}
$$

и $\psi^{*} \in \widetilde{T}_{g, k, m+b}$.

3. Для подгрупшы $\widetilde{\gamma} \subset \gamma=\gamma_{g, n}$ положим

$$
\begin{aligned}
\widetilde{\operatorname{Mod}}_{g, k, m, b}^{\tilde{\gamma}} & =\left\{\alpha \in \widetilde{\operatorname{Mod}}_{g, k, m+b}^{b} \mid \alpha \widetilde{\gamma}=\widetilde{\gamma}\right\}, \\
\widetilde{\operatorname{Iod}}_{g, k, m, b} & =\widetilde{\operatorname{Mod}}_{g, k, m, b} \cap \widetilde{\operatorname{IMod}}_{g, k, m+b}, \\
\operatorname{Mod}_{g, k, m, b}^{\tilde{\gamma}} & =\widetilde{\operatorname{Mod}}_{g, k, m, b} \backslash \widetilde{\operatorname{IMod}}_{g, k, m, b} .
\end{aligned}
$$

Автоморфизм $\alpha \in \widetilde{\operatorname{IMod}}_{g, k, m, b}^{\widetilde{\gamma}}$ переводит $\psi \in \widetilde{T}_{g, k, m+b}$ в $h^{*} \psi \in \widetilde{T}_{g, k, m+b}$, где $h^{*} \psi(w)=h \psi(w) h^{-1}$ и $h \in \operatorname{Aut}(\Lambda)$. Поэтому группа $\operatorname{Mod}_{g, k, m, b}^{\widetilde{\gamma}}$ действует на $T_{g, k, m+b}$.

Пусть $N(g, n, d)$ - множество подгрупп индекса $d$ группы $\gamma_{g, n}$. На этом множестве действует группа $\operatorname{Mod}_{g, k, m+b}^{b}$. Обозначим через $n(g, k, m, b, d)$ число орбит этого действия.

Пусть $H_{g, k, m}^{b, d}$ - пространство голоморфных морфизмов $(\widetilde{P}, f, P)$ таких, что $P \in$ $H_{g, k, m}$, степень $f$ равна $d$ и число критических значений $f$ равно $b$. 
Теорема 6.1. Пространство $H=H_{g, k, m}^{b, d}$ распадается на $n(g, k, m, b, d)$ компонент связности. Каждая из них является классом топологической эквивалентности голоморфных морфизмов и гомеоморфна $T_{g, k, m+b} / \widetilde{\operatorname{Mod}}_{g, k, m, b}{ }_{\gamma}$, әде $\operatorname{Mod}_{g, k, m, b}^{\widetilde{\gamma}}$ действует дискретно.

ДоКАЗАТЕЛЬСТво. Согласно леммам 6.4 и 6.3 каждая компонента связности пространства $H$ имеет вид

$$
H^{t}=\left\{\left(P_{\widetilde{\gamma}}^{\psi}, f_{\widetilde{\gamma}}^{\psi}, P^{\psi}\right)^{b} \mid \psi \in \widetilde{T}_{g, k, m+b}\right\}
$$

и число таких компонент равно $n(g, k, m, b, d)$.

Рассмотрим отображение $\widetilde{\Phi}: \widetilde{T}_{g, k, m+b} \rightarrow H^{t}$, где $\widetilde{\Phi}(\psi)=\left(P_{\widetilde{\gamma}}^{\psi}, f_{\widetilde{\gamma}}^{\psi}, P^{\psi}\right)^{b}$. Согласно лемме 6.2 она индуцирует отображение $\Phi: T_{g, k, m+b} \rightarrow H^{t}$. Нетрудно видеть, что слой отображения $\Phi$ состоит из орбиты групшы $\operatorname{Mod}_{g, k, m, b}^{\widetilde{\gamma}}$. Дискретность действия $\operatorname{Mod}_{g, k, m, b}^{\widetilde{\gamma}}$ следует из дискретности действия $\operatorname{Mod}_{g, k, m, b}$ на $T_{g, k, m+b}$ (теорема 5.1).

СлЕДСТВИЕ 6.1. Пространство всех голоморфнъх морфизмов фиксированного топологического типа связано и гомеоморфно $\mathbb{R}^{s} \backslash \operatorname{Mod}$, где Mod - дискретная әруппа.

ЗАмЕчАНИЕ. Частные случаи этой теоремы были известны ранее: 1) для многочленов Лорана обшего положения [32]; 2) для мероморфных функций обшего положения (вместе с описанием группы Mod [36]; 3) для $g=0$ [37]; 4) для компактных поверхностей [38].

\section{$\S$ 7. Поднятие фуксовых групп на $S L(2, \mathbb{R})$}

1. Для исследования спинорных расслоений и суперримановых поверхностей нам понадобится решение следующей задачи. Пусть

$$
\Gamma \subset \operatorname{Aut}_{1}(\Lambda) \cup \operatorname{Aut}_{2}(\Lambda) \subset \operatorname{Aut}(\Lambda)=P S L(2, \mathbb{R})
$$

- конечно порожденная фуксова группа. Как описать все ее поднятия на $S L(2, \mathbb{R})$, т.е. подгруппы $\Gamma^{*} \subset S L(2, \mathbb{R})$, на которых естественная проекция $J: S L(2, \mathbb{R}) \rightarrow P S L(2, \mathbb{R})$ индуцирует изоморфизм $\Gamma^{*} \rightarrow \Gamma$.

Для начала положим

$$
\operatorname{Aut}_{i}^{*}=J^{-1}\left(\operatorname{Aut}_{i}(\Lambda)\right) \subset S L(2, \mathbb{R}) \quad(i=1,2)
$$

и свяжем с каждой матрищей $C \in \mathrm{Aut}_{i}^{*}$ число $\sigma(C) \in\{1,-1\}$, однозначно определяемое следуюшим условием: матрица $C$ сопряжена матрище

$$
\sigma(C)\left(\begin{array}{cc}
\lambda & 0 \\
0 & \lambda^{-1}
\end{array}\right), \quad \lambda>0
$$

(если $C \in \mathrm{Aut}_{2}^{*}$ ), или матрище

$$
\sigma(C)\left(\begin{array}{ll}
1 & 1 \\
0 & 1
\end{array}\right)
$$

(если $C \in \mathrm{Aut}_{1}^{*}$ ). Таким образом, знак $\sigma(C)$ совпадает со знаком следа матришы $C$.

Лемма 7.1. Пусть $C_{1}, C_{2}, C_{3} \in S L(2, \mathbb{R})$ maкuе, что $\left\{J\left(C_{1}\right), J\left(C_{2}\right), J\left(C_{3}\right)\right\}$ последовательный набор типа $(0, k, m)$. Тогда $C_{1} C_{2} C_{3}=1$, если и только если $\sigma\left(C_{1}\right) \sigma\left(C_{2}\right) \sigma\left(C_{3}\right)=-1$. 
ДокАЗАТЕльство. Предположим сначала, что $C_{1}, C_{2} \in \mathrm{Aut}_{2}^{*}$. После сопряжения пары $\left(C_{1}, C_{2}\right)$ матрищей из $S L(2, \mathbb{R})$ можно считать, что

$$
C_{1}=\frac{\sigma\left(C_{1}\right)}{\sqrt{\lambda_{1}}}\left(\begin{array}{cc}
\lambda_{1} & 0 \\
0 & 1
\end{array}\right)
$$

и

$$
C_{2}=\frac{\sigma\left(C_{2}\right)}{\sqrt{\lambda_{2}}(\alpha-\beta)}\left(\begin{array}{cc}
\left(\lambda_{2} \alpha-\beta\right), & \left(1-\lambda_{2}\right) \alpha \beta \\
\left(\lambda_{2}-1\right), & \left(\alpha-\lambda_{2} \beta\right)
\end{array}\right),
$$

где $\lambda_{1}, \lambda_{2}>1, \alpha<\beta$. Следовательно,

$$
C_{1} C_{2}=\frac{\sigma\left(C_{1}\right) \sigma\left(C_{2}\right)}{\sqrt{\lambda_{1} \lambda_{2}}(\alpha-\beta)}\left(\begin{array}{cc}
\lambda_{1}\left(\lambda_{2} \alpha-\beta\right), & \lambda_{1}\left(1-\lambda_{2}\right) \alpha \beta \\
\left(\lambda_{2}-1\right), & \left(\alpha_{2}-\lambda_{2} \beta\right)
\end{array}\right) .
$$

Характеристическое уравнение этой матрищы имеет вид

$$
x^{2}-\left(\lambda_{2} \beta-\alpha-\lambda_{1} \beta+\lambda_{1} \lambda_{2} \alpha\right) x+\lambda_{1} \lambda_{2}(\alpha-\beta)^{2}=0
$$

Его корни имеют одинаковьй знак, совпадающий со знаком числа

$$
\lambda_{2} \beta-\alpha-\lambda_{1} \beta+\lambda_{1} \lambda_{2} \alpha \text {. }
$$

Согласно лемме 3.2

$$
0<\left(\frac{\sqrt{\lambda_{1}}+\sqrt{\lambda_{2}}}{1+\sqrt{\lambda_{1} \lambda_{2}}}\right)^{2} \beta \leqslant \alpha<\beta<\infty
$$

Следовательно,

$$
\alpha \geqslant\left(\frac{\sqrt{\lambda_{1}}+\sqrt{\lambda_{2}}}{\sqrt{\lambda_{1} \lambda_{2}}+1}\right)^{2} \beta \geqslant\left(\frac{\sqrt{\lambda_{1}}-\sqrt{\lambda_{2}}}{\sqrt{\lambda_{1} \lambda_{2}}-1}\right)^{2} \beta>\frac{\lambda_{1}+\lambda_{2}-2}{\lambda_{1} \lambda_{2}-1} \beta,
$$

откуда

$$
\lambda_{2} \beta-\alpha-\lambda_{1} \beta+\lambda_{1} \lambda_{2} \alpha>2\left(\lambda_{2}-1\right) \beta>0 .
$$

Таким образом,

$$
\sigma\left(C_{1} C_{2}\right)=\sigma\left(C_{1}\right) \sigma\left(C_{2}\right) \operatorname{sign}(\alpha-\beta)=-\sigma\left(C_{1}\right) \sigma\left(C_{2}\right)
$$

и условие $C_{3}^{-1}=C_{1} C_{2}$ эквивалентно

$$
\sigma\left(C_{3}\right)=\sigma\left(C_{3}^{-1}\right)=\sigma\left(C_{1} C_{2}\right)=-\sigma\left(C_{1}\right) \sigma\left(C_{2}\right) .
$$

Аналогично, если $C_{1} \in \mathrm{Aut}_{2}^{*}, C_{2} \in \mathrm{Aut}_{1}^{*}$, то после сопряжения элементом из $S L(2, \mathbb{R})$ можно считать, что

$$
C_{1}=\frac{\sigma\left(C_{1}\right)}{\sqrt{\lambda}}\left(\begin{array}{ll}
\lambda & 0 \\
0 & 1
\end{array}\right)
$$

и

$$
C_{2}=\sigma\left(C_{2}\right)\left(\begin{array}{cc}
1-\alpha \gamma, & a^{2} \gamma \\
-\gamma, & 1+a \gamma
\end{array}\right)
$$


где $\lambda>1, \gamma>0$. Следовательно,

$$
C_{1} C_{2}=\frac{\sigma\left(C_{1}\right) \sigma\left(C_{2}\right)}{\sqrt{\lambda}}\left(\begin{array}{cc}
\lambda(1-a \gamma), & \lambda a^{2} \gamma \\
-\gamma, & 1+a \gamma
\end{array}\right) .
$$

Характеристическое уравнение этой матрищы имеет вид

$$
x^{2}-[\lambda(1-a \gamma)+(1+a \gamma)]+\lambda a^{2} \gamma^{2}=0 .
$$

Его корни имеют одинаковьй знак, совпадающий со знаком числа $\lambda(1-a \gamma)+(1+a \gamma)$. Согласно лемме 3.3

$$
a \gamma \geqslant \frac{\sqrt{\lambda}+1}{\sqrt{\lambda}-1}=\frac{(\sqrt{\lambda}+1)^{2}}{\lambda-1}
$$

откуда

$$
\lambda(1-a \gamma)+(1+a \gamma)=(1+\lambda)+a \gamma(1-\lambda) \leqslant(1+\lambda)-(\sqrt{\lambda}+1)^{2}<0 .
$$

Таким образом,

$$
\sigma\left(C_{1} C_{2}\right)=-\sigma\left(C_{1}\right) \sigma\left(C_{2}\right)
$$

и условие $C_{3}^{-1}=C_{1} C_{2}$ эквивалентно

$$
\sigma_{1}\left(C_{1}\right) \sigma\left(C_{2}\right) \sigma\left(C_{3}\right)=-1 .
$$

Приведенные рассуждения доказывают лемму в случае $k>0$. Если $k=0$, то после сопряжения можно считать, что

$$
C_{1}=\sigma\left(C_{1}\right)\left(\begin{array}{ll}
1 & 1 \\
0 & 1
\end{array}\right), \quad C_{2}=\sigma\left(C_{2}\right)\left(\begin{array}{ll}
-3 & 4 \\
-4 & 5
\end{array}\right) .
$$

Откуда

$$
\sigma\left(C_{1} C_{2}\right)=-\sigma\left(C_{1}\right) \sigma\left(C_{2}\right) .
$$

Лемма 7.2. Пусть $A, B, C \in S L(2, \mathbb{R})$ maкue, что $\{J(A), J(B), J(C)\}-$ noследовательный набор типа $(1,1,0)$. Тогда $[A, B] C=1$, если и только если $\sigma(C)=-1$. Кроме того, $\sigma(A B)=\sigma(A) \sigma(B)$.

ДокАЗАТЕЛЬСТво. По определению $\sigma(A)=\sigma\left(B A^{-1} B^{-1}\right)$ и $\left\{J(A), J\left(B A^{-1} B^{-1}\right)\right.$, $J(C)\}$ - последовательньй набор типа $(0,3,0)$. Поэтому первое утверждение леммы 7.2 следует из леммы 7.1. Докажем, что $\sigma(A, B)=\sigma(A) \sigma(B)$. После сопряжения элементом из $S L(2, \mathbb{R})$ можно считать, что

и

$$
A=\frac{\sigma(A)}{\sqrt{\lambda_{A}}}\left(\begin{array}{ll}
\lambda & 0 \\
0 & 1
\end{array}\right)
$$

$$
B=\frac{\sigma(B)}{\sqrt{\lambda_{2}}(\alpha-\beta)}\left(\begin{array}{cc}
\lambda_{2} \alpha-\beta, & \left(1-\lambda_{2}\right) \alpha \beta \\
\lambda_{2}-1, & \alpha-\lambda_{2} \beta
\end{array}\right),
$$

где $\lambda_{1}, \lambda_{2}>1, \beta<0<\alpha$ (см. рис. 7.1$)$.

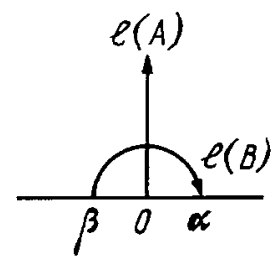

Рис. 7.1 
Следовательно,

$$
A B=\frac{\sigma(A) \sigma(B)}{\sqrt{\lambda_{1} \lambda_{2}}(\alpha-\beta)}\left(\begin{array}{cc}
\lambda_{1}\left(\lambda_{2} \alpha-\beta\right), & \lambda_{1}\left(1-\lambda_{2}\right) \alpha \beta \\
\lambda_{2}-1, & \alpha-\lambda_{2} \beta
\end{array}\right)
$$

Корни характеристического уравнения этой матрицы имеют одинаковьй знак, совпадающий со знаком

$$
\lambda_{1}\left(\lambda_{2} \alpha-\beta\right)+\left(\alpha-\lambda_{2} \beta\right)>0 \text {. }
$$

Таким образом,

$$
\sigma(A B)=\sigma(A) \sigma(B)
$$

Teopema 7.1. Пусть $\left\{A_{i}, B_{i}(i=1, \ldots, g), C_{i}(i=g+1, \ldots, n)\right\} \subset S L(2, \mathbb{R}) u$ $\left\{J\left(A_{i}\right), J\left(B_{i}\right)(i=1, \ldots, g), J\left(C_{i}\right)(i=g+1, \ldots, n)\right\}$ - последовательный набор muna $(g, k, m)$. Тогда

$$
\prod_{i=1}^{g}\left[A_{i}, B_{i}\right] \prod_{i=g+1}^{n} C_{i}=1
$$

если и только если или $n=g$, или

$$
\prod_{i=g+1}^{n} \sigma\left(C_{i}\right)=(-1)^{n-g}
$$

ДокАЗАТЕЛЬСТво. Согласно лемме $7.2 \sigma\left(\left[A_{i}, B_{i}\right]\right)=-1$. Поэтому утверждение теоремы достаточно доказать для $g=0$. В этом случае воспользуемся индукцией по $n$. Для $n=3$ утверждение следует из леммы 7.1. Шаг индукции также следует из леммы 7.1 , если применить ее к набору $\left\{C_{1} \cdots C_{n-2}, C_{n-1}, C_{n}\right\}$.

2. Свяжем теперь с поднятием фуксовой групшы

$$
\Gamma \subset \operatorname{Aut}_{2}(\Lambda) \cup \operatorname{Aut}_{1}(\Lambda)
$$

функцию

$$
H_{1}\left(\Lambda / \Gamma, \mathbb{Z}_{2}\right) \rightarrow \mathbb{Z}_{2}
$$

где $\mathbb{Z}_{2}=\mathbb{Z} / 2 \mathbb{Z}=\{0,1\}$. Итак, пусть $P=\Lambda / \Gamma$ и $\Gamma^{*} \subset S L(2, \mathbb{R})$ - подгруппа, на которой $J$ индуцирует изоморфизм $\widetilde{J}: \Gamma^{*} \rightarrow \Gamma$. При выбранной точке $q \in \Lambda$ униформизация $\Phi: \Lambda \rightarrow P$ индуцирует изоморфизм $\Phi_{q}: \Gamma \rightarrow \pi_{1}(P, p)$, где $p=\Phi(q)$ (см. $\left.\S 2\right)$. Построим функцию $\widetilde{\omega}: \pi_{1}(P, p) \rightarrow \mathbb{Z}_{2}$, положив

$$
\widetilde{\omega}(c)=\left\{\begin{array}{l}
0, \text { если } \sigma\left(\widetilde{J}^{-1}\left(\Phi_{q}^{-1}(c)\right)\right)=-1, \\
1, \text { если } \sigma\left(\widetilde{J}^{-1}\left(\Phi_{q}^{-1}(c)\right)\right)=1 .
\end{array}\right.
$$

Обозначим через $(\cdot, \cdot): H_{1}\left(P, \mathbb{Z}_{2}\right) \times H_{2}\left(P, \mathbb{Z}_{2}\right) \rightarrow \mathbb{Z}_{2}$ билинейную форму индекса пересечения в $H_{1}\left(P, \mathbb{Z}_{2}\right)$. Естественная проекция $\pi_{1}(P, p) \rightarrow H_{1}\left(P, \mathbb{Z}_{2}\right)$ переносит $(\cdot, \cdot)$ на $\pi_{1}(P, p)$. 
ЛЕмма 7.3. Пусть $\widetilde{c}_{1}, \widetilde{c}_{2}, \widetilde{c}_{3} \subset P$ - простые контуры такие, ито $\widetilde{c}_{i} \cap \widetilde{c}_{j}=p$, и отвечающие им $c_{i} \in \pi_{1}(P, p)$ такие, что $c_{i} \neq 1$ и $c_{1} c_{2} c_{3}=1$. Тогда $\widetilde{\omega}\left(c_{3}\right)=$ $\widetilde{\omega}\left(c_{1}\right)+\widetilde{\omega}\left(c_{2}\right)+\left(c_{1}, c_{2}\right)$.

ДокАЗАТЕЛЬСтво. Согласно теореме 2.1 сдвиги $\Phi_{q}^{-1}\left(c_{i}\right)$ образуют последовательньй набор типа $(0, k, m)$ или $(2,1,0)$. В первом случае $\left(c_{1}, c_{2}\right)=0$ и $\widetilde{\omega}\left(c_{3}\right)=\widetilde{\omega}\left(c_{1}\right)+\widetilde{\omega}\left(c_{2}\right)$ ввиду леммы 7.1. Во втором случае $\left(c_{1}, c_{2}\right)=1$ и $\widetilde{\omega}\left(c_{3}\right)=\widetilde{\omega}\left(c_{1}\right)+\widetilde{\omega}\left(c_{2}\right)+1$ ввиду леммы 7.2.

Лемма 7.4. Пусть $d_{1}, d_{2} \in \pi_{1}(P, p)$ представляют один и тот же әлемент группьь $H_{1}\left(P, \mathbb{Z}_{2}\right)$. Тогда $\widetilde{\omega}\left(d_{1}\right)=\widetilde{\omega}\left(d_{2}\right)$.

ДокАЗАтельство. Рассмотрим стандартную систему образующих

$$
v=\left\{a_{i}, b_{i}(i=1, \ldots, g), c_{i}(i=g+1, \ldots, n)\right\}
$$

группы $\pi_{1}(P, p)$. Рассмотрим функцию $\omega_{v}: H_{1}\left(P, \mathbb{Z}_{2}\right) \rightarrow \mathbb{Z}_{2}$, совпадаюшую с $\widetilde{\omega}$ на $v$ и удовлетворяюшую условию $\omega_{v}(a+b)=\omega_{v}(a)+\omega_{v}(b)+(a, b)$ для любых $a, b \in$ $H_{1}\left(P, \mathbb{Z}_{2}\right)$. Эти условия однозначно определяют $\omega_{v}$. Рассмотрим скручивания Дена [39], т.е. преобразования одного из трех типов:

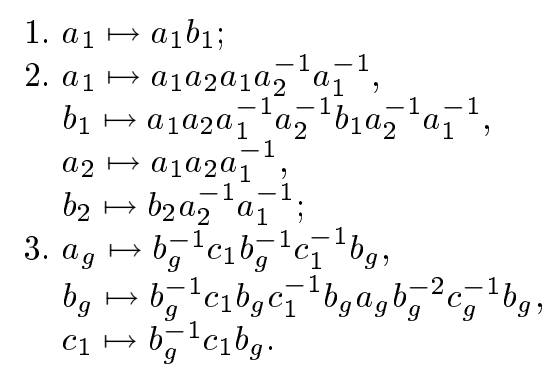

Они переводят $v$ в другие стандартные системы образующих $\widetilde{v}$. Из леммы 7.3 следует, что $\omega_{\widetilde{v}}=\omega_{v}$. С другой стороны, при помощи последовательности скручивания Дена и перенумераций можно получить из $v$ любую стандартную систему образующих и, в частности, такую, что $a_{1}$ - любой наперед заданньй простой контур, не лежащий в ядре $(\cdot, \cdot)$, a $c_{g+1}-$ любой наперед заданный простой контур, гомологичный дыре или проколу. Таким образом, $\widetilde{\omega}=\omega_{v} \eta$, где $\eta: \pi_{1}(P, p) \rightarrow H_{1}\left(P, \mathbb{Z}_{2}\right)$ - естественная проекция.

Согласно лемме 7.4 функция $\widetilde{\omega}$ индуцирует функцию $\omega: H_{1}\left(P, \mathbb{Z}_{2}\right) \rightarrow \mathbb{Z}_{2}$. Из леммы 7.3 сразу следует

СлЕДСТвИе 7.1. $\omega(a+b)=\omega(a)+\omega(b)+(a, b)$.

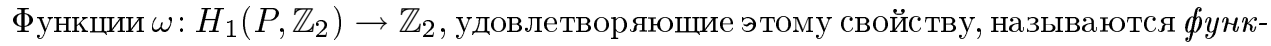
ииями Аpфa [40]. Таким образом, наша конструкция сопоставляет всякому поднятию $\Gamma^{*}$ групшы $\Gamma$ некоторую функцию Арфа $\omega_{\Gamma^{*}}: H_{1}\left(\Lambda / \Gamma, \mathbb{Z}_{2}\right) \rightarrow \mathbb{Z}_{2}$.

TeOPEмa 7.2 [41]. Соответствие $\Gamma^{*} \mapsto \omega_{\Gamma^{*}}$ является взаимно однозначныцм соответствием между поднятиями группь Г и функииями Арфа на $H_{1}\left(\Lambda / \Gamma, \mathbb{Z}_{2}\right)$. 
ДокАЗАТЕЛЬСТво. Из конструкции очевидно, что разньм поднятиям отвечают разные функции Арфа. Сопоставим теперь произвольной функции Арфа $\omega$ поднятие $\Gamma^{*}$ такое, что $\omega_{\Gamma^{*}}=\omega$. Для этого рассмотрим произвольный последовательный набор

$$
V=\left\{A_{i}, B_{i}(i=1, \ldots, g), C_{i}(i=g+1, \ldots, n)\right\}=\left\{D_{1}, \ldots, D_{g+n}\right\},
$$

порождающий Г. Рассмотрим матрицы

$$
V^{*}=\left\{D_{1}^{*}, \ldots, D_{g+n}^{*}\right\} \in S L(2, \mathbb{R}),
$$

однозначно определенные условиями

$$
J\left(D_{i}^{*}\right)=D_{i} \quad \text { и } \quad \sigma\left(D_{i}^{*}\right)=\left\{\begin{aligned}
-1, & \text { если } \omega\left(\Phi_{q}\left(D_{i}\right)\right)=0, \\
1, & \text { если } \omega\left(\Phi_{q}\left(D_{i}\right)\right)=1 .
\end{aligned}\right.
$$

Согласно лемме 7.3 и теореме 7.1

$$
\prod_{i=1}^{g}\left[A_{i}^{*}, B_{i}^{*}\right] \prod_{i=g+1}^{n} C_{i}^{*}=1
$$

Ввиду того, что

$$
\prod_{i=1}^{g}\left[A_{i}, B_{i}\right] \prod_{i=g+1}^{n} C_{i}=1
$$

- единственное определяющее соотношение в $Г$, отсюда следует, что ограничение гомоморфизма $J$ на группу $\Gamma^{*}$, порожденную $V^{*}$, индуцирует изоморфизм $\Gamma^{*} \rightarrow \Gamma$. По построению $\omega_{\Gamma^{*}}=\omega$.

\section{§ 8. Топологическая классификация функций Арфа и пар функций Арфа}

В предыдушем параграфе мы свели описание поднятий фуксовых групп к функциям Арфа, т.е. отображениям $\omega: H_{1}\left(P, \mathbb{Z}_{2}\right) \rightarrow \mathbb{Z}_{2}$ таким, что

$$
\omega(a+b)=\omega(a)+\omega(b)+(a, b)
$$

где $\mathbb{Z}_{2}=\mathbb{Z} / 2 \mathbb{Z}_{\text {и }}(a, b) \in \mathbb{Z}_{2}$ - индекс пересечения $a$ и $b$ в $H_{1}\left(P, \mathbb{Z}_{2}\right)$. Автогомеоморфизмы $\varphi: P \rightarrow P$ действуют на множестве функций Арфа. Цель этого параграфа - найти орбиты этого действия.

Итак, пусть $P$ - поверхность типа $(g, k, m)$. Базис

$$
v=\left\{a_{i}, b_{i}(i=1, \ldots, g), c_{i}(i=g+1, \ldots, n)\right\}
$$

группы $H_{1}\left(P, \mathbb{Z}_{2}\right)$ назовем стандартнылм, если он является образом стандартной системы образующих

$$
v^{\prime}=\left\{a_{i}^{\prime}, b_{i}^{\prime}(i=1, \ldots, g), \quad c_{i}^{\prime}(i=g+1, \ldots, n)\right\} \in \pi_{1}(P, p)
$$

при естественной проекции $\pi_{1}(P, p) \rightarrow H_{1}\left(P, \mathbb{Z}_{2}\right)$. 
Рассмотрим группу автогомеоморфизмов $\operatorname{Mod}(P)$ поверхности $P$, переводяших дыры в дыры и проколы в проколы. Она порождает группу $A(P)$, эффективно действуюшую на множестве функций Арфа. Элемент группы $A(P)$ однозначно определяется образом стандартного базиса $v$. Образуюшие Дена [39] групшы $\operatorname{Mod}(P)$ определяют образуюшие группы $A(P)$. Они разделяются на 5 типов и однозначно определяются тем, в какой стандартный базис

$$
\widetilde{v}=\left\{\widetilde{a}_{i}, \widetilde{b}_{i}(i=1, \ldots, g), \widetilde{c}_{i}(i=g+1, \ldots, n)\right\} \subset H_{1}\left(P, \mathbb{Z}_{2}\right)
$$

переходит фиксированньй стандартный базис

$$
v=\left\{a_{i}, b_{i}(i=1, \ldots, g), c_{i}(i=g+1, \ldots, n)\right\} \subset H_{1}\left(P, \mathbb{Z}_{2}\right) .
$$

Эти образующие имеют один из следующих 5 видов:

(1) $\widetilde{a}_{i}=a_{i}+b_{i} ; \quad$ (2) $\widetilde{a}_{i}=b_{i}, \widetilde{b}_{i}=a_{i} ; \quad(3) \widetilde{a}_{i}=a_{i}+a_{j}, \widetilde{b}_{j}=b_{j}, \widetilde{a}_{i}=a_{i}, \widetilde{b}_{i}=b_{i}+b_{j}$;

$$
\text { (4) } \tilde{a}_{i}=a_{i}+c_{r} ; \quad \text { (5) } \widetilde{c}_{i}=c_{j}, \widetilde{c}_{j}=c_{i} \text {, }
$$

где $\widetilde{a}_{k}=a_{k}, \widetilde{b}_{k}=b_{k}, \widetilde{c}_{k}=c_{k}$ при $k \neq i, j$.

Положим $\delta=\delta(P, \omega)=0$, если существует стандартньй базис $v$ такой, что

$$
\sum_{i=1}^{g} \omega\left(a_{i}\right) \omega\left(b_{i}\right) \equiv 0 \quad(\bmod 2)
$$

В остальных случаях положим $\delta=1$.

Множество дыр $c_{g+1}, \ldots, c_{g+k}$ делится на 2 множества

$$
D_{0}=\left\{c_{i} \mid \omega\left(c_{i}\right)=0\right\}, \text { и } D_{1}=\left\{c_{i} \mid \omega\left(c_{i}\right)=1\right\} .
$$

Обозначим через $k_{\alpha}=k_{\alpha}(P, \omega)$ мошность множества $D_{\alpha}$. Аналогично проколы $c_{g+k+1}, \ldots, c_{n}$ делятся на 2 множества вида

$$
E_{\alpha}=\left\{c_{i} \mid \omega\left(c_{i}\right)=\alpha\right\}
$$

Пусть $m_{\alpha}(\alpha=0,1)$ - мошность множества $E_{\alpha}$. Типом функиии $A p \phi a(P, \omega)$ назовем набор $\left(g, \delta, k_{0}, k_{1}, m_{0}, m_{1}\right)$. Положим $n_{\alpha}=k_{\alpha}+m_{\alpha}$.

Лемма 8.1. Набор $\left(g, \delta, k_{\alpha}, m_{\alpha}\right)$ является типом некоторой функции Арфа, если и только если

$$
\text { 1) } k_{1}+m_{1} \equiv 0 \quad(\bmod 2) ; \quad \text { 2) } \delta=0 \quad n p u \quad n_{1}>0 .
$$

В этом случае существует стандартный базис $v$ такой, что $\omega\left(a_{i}\right)=\omega\left(b_{i}\right)=0$ для $i>1, \omega\left(a_{1}\right)=\omega\left(b_{1}\right)=\delta, \omega\left(c_{i}\right)=0$ nри $g+1 \leqslant i \leqslant g+k_{0}$ u $g+k_{0}+k_{1}+1$ $\leqslant i \leqslant g+k_{0}+k_{1}+m_{1}$. 
ДокаЗАТЕльСтво. Условие $k_{1}+m_{1} \equiv 0(\bmod 2)$ следует из

$$
\sum_{i=g+1}^{n} \omega\left(c_{i}\right)=\omega\left(\sum_{i=g+1}^{n} c_{i}\right)=\omega(0)=0 .
$$

Докажем теперь, что существует стандартный базис $v$ такой, что $\omega\left(a_{i}\right)=\omega\left(b_{i}\right)=0$ при $i>1$ и $\omega\left(a_{1}\right)=\omega\left(b_{1}\right)=0$ при $n_{1}>0$. Нетрудно видеть, что, используя образующие (1)-(3), можно перейти от произвольного стандартного базиса $v$ к стандартному базису $v$ такому, что $\omega\left(a_{i}\right)=\omega\left(b_{i}\right)=0$ при $i>1$ и $\omega\left(a_{1}\right)=\omega\left(b_{1}\right)$. Если при этом $\omega\left(a_{1}\right)=\omega\left(b_{1}\right)=\omega\left(c_{i}\right)=1$, то преобразование $\widetilde{a}_{1}=a_{1}+c_{i}, \widetilde{b}_{1}=b_{1}+c_{i}$ дает нужный базис. Если $n_{1}=0$, то преобразования (1)-(5) сохраняют четность суммы

$$
\sum_{i=1}^{g} \omega\left(a_{i}\right) \omega\left(b_{i}\right)
$$

и, следовательно, тип $\left(g, 1, k_{0}, 0, m_{0}, 0\right)$ существует.

Пусть теперь $\left(P, \omega_{1}, \omega_{2}\right)$ - пара функций Арфа, причем $\omega_{1} \neq \omega_{2}$. Типом этой пары назовем набор $\left(g, \delta_{1}, \delta_{2}, k_{\alpha \beta}, m_{\alpha \beta}\right)$, где $\delta_{i}=\delta\left(P, \omega_{i}\right), k_{\alpha \beta}$ - число дыр $c_{i}$ таких, что $\omega_{1}\left(c_{i}\right)=\alpha, \omega_{2}\left(c_{i}\right)=\beta$, и $m_{\alpha \beta}$ - число проколов $c_{i}$ таких, что $\omega_{1}\left(c_{i}\right)=\alpha, \omega_{2}\left(c_{i}\right)=\beta$, где $\alpha, \beta \in\{0,1\}$. Положим $n_{\alpha \beta}=k_{\alpha \beta}+m_{\alpha \beta}$.

Лемма 8.2. Набор $\left(g, \delta_{1}, \delta_{2}, k_{\alpha \beta}, m_{\alpha \beta}\right)$ является типом некоторой пары функиий Арфа, если и только если наборь $\left(g, \delta_{1}, k_{\alpha 0}+k_{\alpha 1}, m_{\alpha 0}+m_{\alpha 1}\right) u\left(g, \delta_{2}, k_{0 \alpha}+k_{1 \alpha}\right.$, $\left.m_{0 \alpha}+m_{1 \alpha}\right)$ являются типами некоторых функций Арфа. В этом случае существует стандартный базис v такой, что:

1) если $n_{10}+n_{01}>0$, то $\omega_{j}\left(a_{1}\right)=\omega_{j}\left(b_{1}\right)=\delta_{j}, \omega_{j}\left(a_{i}\right)=\omega_{j}\left(b_{i}\right)=0$, где $j=1,2, i>1$

2) если $n_{10}=n_{01}=0$ и $n_{11}>0$, то $\omega_{j}\left(a_{i}\right)=\omega_{j}\left(b_{i}\right)=0$ при $j=1,2, i>1 u$ $\omega_{1}\left(b_{1}\right)=\omega_{2}\left(a_{1}\right)=\omega_{2}\left(b_{1}\right)=0, \omega_{1}\left(a_{1}\right)=1$

3) если $n_{01}=n_{10}=n_{11}=0$, то $\omega_{1}\left(a_{1}\right)+\omega_{2}\left(a_{1}\right)=1, \omega_{1}\left(a_{2}\right)=\omega_{1}\left(b_{2}\right)=$ $\omega_{2}\left(a_{2}\right)=\omega_{2}\left(b_{2}\right)=\varepsilon, \omega_{1}\left(b_{1}\right)=\omega_{2}\left(b_{1}\right)=\delta\left(\right.$ əде $\varepsilon=0$, eсли $\delta=1, u \omega_{1}\left(a_{1}\right)=1$, если $\delta=0)$ и $\omega_{k}\left(a_{i}\right)=\omega_{k}\left(b_{i}\right)=0$ при $i>2$.

ДокАЗАТельСтво. Первое утверждение очевидно. Пусть $\left(P, \omega_{1}, \omega_{2}\right)$ - пара несовпадаюших функций Арфа. Рассмотрим эпиморфизм $\omega=\left(\omega_{1}+\omega_{2}\right): H_{1}\left(P, \mathbb{Z}_{2}\right) \rightarrow \mathbb{Z}_{2}$. Используя преобразования (1)-(5), нетрудно перейти от произвольного базиса к базису

$$
v=\left\{a_{i}, b_{i}, c_{j}\right\} \subset H_{1}\left(P, \mathbb{Z}_{2}\right)
$$

такому, что

$$
\omega\left(b_{1}\right)=\omega\left(a_{i}\right)=\omega\left(b_{i}\right)=0 \text { для } i>1
$$

и

$$
\omega\left(a_{1}\right)=0 \text { при } n_{01}+n_{10}>0 .
$$

Пусть $n_{01}+n_{10}>0$. Тогда $\omega_{1}\left(a_{i}\right)=\omega_{2}\left(a_{i}\right)$ и $\omega_{1}\left(b_{i}\right)=\omega_{2}\left(b_{i}\right)$. Перестройки $(1)-(3)$ дают базис $v$ такой, что

$$
\omega_{k}\left(a_{i}\right)=\omega_{k}\left(b_{i}\right)=0 \text { для } i>1
$$


и

$$
\omega_{1}\left(a_{1}\right)=\omega_{1}\left(b_{1}\right)=\omega_{2}\left(a_{1}\right)=\omega_{2}\left(b_{1}\right) .
$$

Используя, если необходимо, перестройки $\widetilde{a}_{1}=a_{1}+c_{k}$ и $\widetilde{b}_{1}=b_{1}+c_{k}$, получаем $\omega_{k}\left(\widetilde{a}_{1}\right)=\omega_{k}\left(\widetilde{b}_{1}\right)=\delta_{k}$. Пусть теперь $n_{01}=n_{10}=0$ и $b \subset P-$ простой контур, представляюший $b_{1}$. Тогда функции $\omega_{1}$ и $\omega_{2}$ равны на $P \backslash b$, и согласно лемме 8.1 сушествует базис $v \in H_{1}\left(P, \mathbb{Z}_{2}\right)$ такой, что:

1) $\omega_{k}\left(a_{i}\right)=\omega_{k}\left(b_{i}\right)=0$ при $i>2$;

2) $\omega_{1}\left(a_{2}\right)=\omega_{1}\left(b_{2}\right)=\omega_{2}\left(a_{2}\right)=\omega_{2}\left(b_{2}\right)=\varepsilon, \omega_{1}\left(b_{1}\right)=\omega_{2}\left(b_{1}\right)=\delta, \omega_{1}\left(a_{1}\right)+$ $\omega_{2}\left(a_{1}\right)=1$ и $\varepsilon=0$, если $\delta=1$ или $n_{11}>0$.

Если $n_{11}>0$, то при подходящем $k$ перестройка $\widetilde{b}_{1}=b_{1}+c_{k}$ дает $\delta=0$. Если $\delta=0$, перестройка $\widetilde{a}_{1}=a_{1}+b_{1}$ дает $\omega_{1}\left(\widetilde{a}_{1}\right)=1$.

Два набора функций Арфа $\left(\omega_{1}, \ldots, \omega_{n}\right)$ и $\left(\omega_{1}^{\prime}, \ldots, \omega_{n}^{\prime}\right)$ на $P$ назовем топологически әквивалентными, если сушествует гомеоморфизм $\alpha \in \operatorname{Mod}(P)$ такой, что $\omega_{i}^{\prime}=\omega_{i} \alpha$.

Теорема 8.1 [42], [43]. Функиии Арфа и пары функиий Арфа топологически эквивалентны, если и только если они имеют одинаковый топологический тип.

ДокАЗАТЕльство. Непосредственно проверяется, что преобразования (1)-(5) сохраняют топологический тип функций Арфа и пар функций Арфа. Эти преобразования порождают группу $A(P)$. Следовательно, топологический тип не меняется под действием $\alpha \in \operatorname{Mod}(P)$. Пусть теперь $\omega$ и $\omega^{\prime}$ имеют одинаковый топологический тип. Тогда согласно лемме 8.1 сушествуют стандартные базисы $v=\left\{v_{i}\right\}$ и $v^{\prime}=\left\{v_{i}^{\prime}\right\}$ группы $\pi_{1}(P, p)$ такие, что $\omega\left(v_{i}\right)=\omega^{\prime}\left(v_{i}^{\prime}\right)$. Рассмотрим $\alpha \in \operatorname{Mod}(P)$ такой, что $\alpha\left(v_{i}^{\prime}\right)=v_{i}$. Тогда $\omega^{\prime}=\omega \alpha$ и, значит, $\omega^{\prime}$ и $\omega$ топологически эквивалентны. Аналогично с использованием леммы 8.2 доказывается второе утверждение теоремы 8.1.

Особенно важен случай компактных поверхностей, который мы и рассматриваем до конца этого параграфа. В этом случае согласно нашим определениям топологический тип функции Арфа описьвается элементом $\delta(\omega) \in\{0,1\}$, назьваемьм Арф-инварианmом [44].

Лемма 8.3. Пусть $\left\{a_{i}, b_{i}(i=1, \ldots, g)\right\}$ - произвольный стандартный базис $H_{1}\left(P, \mathbb{Z}_{2}\right)$. Тогда

$$
\delta(\omega) \equiv \sum_{i=1}^{g} \omega\left(a_{i}\right) \omega\left(b_{i}\right) \quad(\bmod 2) .
$$

ДокАЗАТЕльСтво. Правая часть сравнения инвариантна относительно преобразований (1)-(5). С другой стороны, эти преобразования порождают группу, транзитивно действующую на множестве всех стандартных базисов.

Для случая компактных поверхностей теорема 8.1 и лемма 8.2 дают

СЛЕДСТВИЕ 8.1. Функиии Арфа на компактной поверхности топологически әквивалентнь, если и только если их Арф-инварианты совпадают. Пары функций Арфа на компактной поверхности топологически эквивалентны, если и только если их Арф-инварианты совпадают.

В следуюшем параграфе мы докажем аналог этого утверждения для произвольного числа функций Арфа. 


\section{§. Топологическая классификация независимых систем функций Арфа на компактных поверхностях}

Под функцией Арфа в этом параграфе мы понимаем функцию Арфа на фиксированной компактной поверхности $P$ рода $g$. Сумма четного числа таких функций является линейной формой вида $H_{1}\left(P, \mathbb{Z}_{2}\right) \rightarrow \mathbb{Z}_{2}$. Сумма нечетного числа функций Арфа - снова функция Арфа.

Набор функций Арфа

$$
\Omega=\left(\omega_{0}, \omega_{1}, \ldots, \omega_{n}\right)
$$

назовем независимым, если равенство

$$
\sum_{i=0}^{n} \alpha_{i} \omega_{i}=0
$$

где $\alpha_{i} \in\{0,1\}$, влечет $\alpha_{0}=\alpha_{1}=\cdots=\alpha_{n}=0$. Цель этого параграфа - доказать следуюшую теорему.

Tеорема 9.1 [45]. Наборы независимых функиий Арфа $\Omega^{1}=\left(\omega_{0}^{1}, \ldots, \omega_{n}^{1}\right)$ и $\Omega^{2}=$ $\left(\omega_{0}^{2}, \ldots, \omega_{n}^{2}\right)$ топологически эквивалентны, если и только если $\delta\left(\omega_{0}^{1}+\omega_{i}^{1}+\omega_{j}^{1}\right)=$ $\delta\left(\omega_{0}^{2}+\omega_{i}^{2}+\omega_{j}^{2}\right) \partial л я$ в всех $i, j=0,1, \ldots, n$.

Для доказательства рассмотрим $H_{1}\left(P, \mathbb{Z}_{2}\right)$ как векторное пространство $H$ над $\mathbb{Z}_{2}$. Индекс пересечения индуцирует на $H$ невырожденную симметричную билинейную форму $(\cdot, \cdot): H \times H \rightarrow \mathbb{Z}_{2}$ такую, что $(a, a)=0$. Базис

$$
\left\{a_{i}, b_{i}(i=1, \ldots, g)\right\} \subset H
$$

назовем симплектическим, если $\left(a_{i}, a_{j}\right)=\left(b_{i}, b_{j}\right)=0,\left(a_{i}, b_{j}\right)=\delta_{i j}$.

Пусть $V=\left(v_{1}, \ldots, v_{n}\right) \subset H$ - набор линейно независимых векторов. Опишем процедуру "ортогонализации" $V$. Ее результатом будет множество $W=W(V)=$ $\left\{s_{i}, t_{i}(i=1, \ldots, r), s_{i}(i=r+1, \ldots, r+h)\right\}$, которое строится рекурсивно, начиная c $V^{0}=V$.

Переход от

$$
V^{k-1}=\left\{s_{i}, t_{i}(i=1, \ldots, k-1), v_{j_{1}}^{k-1}, \ldots, v_{j_{n_{k-1}}}^{k-1}\right\}
$$

$\mathrm{K}$

$$
V^{k}=\left\{s_{i}, t_{i}(i=1, \ldots, k), v_{j_{1}}^{k}, \ldots, v_{j_{n_{k}}}^{k}\right\}
$$

дается следуюшими правилами.

Если все $v_{i}^{k-1}$ попарно ортогональны (т.е. $\left(v_{i}^{k-1}, v_{j}^{k-1}\right)=0$ ), то положим

$$
W=V^{k-1} .
$$

В противном случае

1) выберем $v_{m}^{k-1}$ с наименьшим номером $i$, не ортогональный хотя бы одному $v_{m}^{k-1}$;

2) выберем $v_{m}^{k-1}$ с наименьшим номером $j$ такой, что $\left(v_{i}^{k-1}, v_{j}^{k-1}\right)=1$;

3) положим $s_{k}=v_{i}^{k-1}, t_{k}=v_{j}^{k-1}$;

4) для $m \neq i, j$ положим $v_{m}^{k}=v_{m}^{k-1}-\left(v_{m}^{k-1}, s_{k}\right) t_{k}+\left(v_{m}^{k-1}, t_{k}\right) s_{k}$. 
ЛЕмма 9.1. Пусть наборы линейно независимых векторов $V=\left(v_{1}, \ldots, v_{n}\right)$ и $V^{\prime}=\left(v_{1}^{\prime}, \ldots, v_{n}^{\prime}\right)$ таковы, что $\left(v_{i}, v_{j}\right)=\left(v_{0}^{\prime}, v_{j}^{\prime}\right)$ для всех $i, j$. Пусть $\omega u \omega^{\prime}-$ функиии Арфа такие, что $\delta(\omega)=\delta\left(\omega^{\prime}\right) u \omega\left(v_{i}\right)=\omega^{\prime}\left(v_{i}^{\prime}\right)$ для $i=1, \ldots, n$. Тогда существует изоморфизм $\varphi: H \rightarrow H$ такой, что $(a, b)=(\varphi(a), \varphi(b)), \omega^{\prime} \varphi=\omega u$ $\varphi\left(v_{i}\right)=\varphi\left(v_{i}^{\prime}\right)$ для всех $a, b \in H, v_{i} \subset V$.

ДокАЗАТЕЛЬСтво. Пусть

$$
W(V)=\left\{s_{i}, t_{i}(i=1, \ldots, r), s_{i}(i=r+1, \ldots, r+h)\right\}
$$

$$
W\left(V^{\prime}\right)=\left\{s_{i}^{\prime}, t_{i}^{\prime}(i=1, \ldots, r), s_{i}^{\prime}(i=r+1, \ldots, r+h)\right\} .
$$

Тогда из $\omega\left(v_{i}\right)=\omega^{\prime}\left(v_{i}^{\prime}\right)$ следует $\omega\left(s_{i}\right)=\omega^{\prime}\left(s_{i}^{\prime}\right), \omega\left(t_{i}\right)=\omega^{\prime}\left(t_{i}^{\prime}\right)$. Кроме того, $\left(s_{i}, s_{j}\right)=$ $\left(t_{i}, t_{j}\right)=\left(s_{i}^{\prime}, s_{j}^{\prime}\right)=\left(t_{i}^{\prime}, t_{j}^{\prime}\right)=0,\left(s_{i}, t_{j}\right)=\left(s_{i}^{\prime}, t_{j}^{\prime}\right)=\delta_{i j}$ и, значит, $W(V)$ и $W\left(V^{\prime}\right)$ можно дополнить до симплектических базисов

$$
\left\{a_{i}, b_{i}(i=1, \ldots, g)\right\}, \quad\left\{a_{i}^{\prime}, b_{i}^{\prime}(i=1, \ldots, g)\right\} .
$$

Используя условие $\delta(\omega)=\delta\left(\omega^{\prime}\right)$ и повторяя рассуждения леммы 8.1 , нетрудно убедиться, что дополняюшие векторы можно выбрать так, чтобы $\omega\left(a_{i}\right)=\omega^{\prime}\left(a_{i}^{\prime}\right)$ и $\omega\left(b_{i}\right)=$ $\omega^{\prime}\left(b_{i}^{\prime}\right)$ для всех $i$. Соответствие $a_{i} \mapsto a_{i}^{\prime}, b_{i} \mapsto b_{i}^{\prime}$ порождает изоморфизм $\varphi: H \rightarrow H$ с нужными свойствами.

Символом * мы будем обозначать двойственные относительно формы $(\cdot, \cdot)$ объекты. В частности, если $\omega^{\prime}$ и $\omega^{\prime \prime}-$ функции Арфа, то $\omega^{\prime}+\omega^{\prime \prime} \in H^{*}$ и $\left(\omega^{\prime}+\omega^{\prime \prime}\right)^{*} \in H$.

Лемма 9.2. Пусть $\left\{\omega_{0}, \omega_{1}, \omega_{2}\right\}-$ функиии Арфа. Тогда

1) $\omega_{0}\left(\left(\omega_{0}+\omega_{1}\right)^{*}\right)=\delta\left(\omega_{0}\right)+\delta\left(\omega_{1}\right)$;

2) $\delta\left(\omega_{0}+\omega_{1}+\omega_{2}\right)=\delta\left(\omega_{0}\right)+\delta\left(\omega_{1}\right)+\delta\left(\omega_{2}\right)+\left(\left(\omega_{0}+\omega_{1}\right)^{*},\left(\omega_{0}+\omega_{2}\right)^{*}\right)$.

ДокАЗАТЕльствО. Пусть $\left\{a_{i}, b_{i}(i=1, \ldots, g)\right\}$ - симплектический базис $H$ такой, что $a_{1}=\left(\omega_{0}+\omega_{1}\right)^{*}$. Тогда $\omega_{1}=\omega_{0}+a_{1}^{*}$ и, следовательно,

$\omega_{1}\left(a_{i}\right)=\omega_{0}\left(a_{i}\right)$ при всех $i, \omega_{1}\left(b_{i}\right)=\omega_{0}\left(b_{i}\right)$ при $i>1$ и $\omega_{1}\left(b_{1}\right)=\omega_{0}\left(b_{1}\right)+1$.

Таким образом,

$$
\delta\left(\omega_{0}\right)+\delta\left(\omega_{1}\right)=\omega_{0}\left(a_{1}\right) \omega_{0}\left(b_{1}\right)+\omega_{1}\left(a_{1}\right) \omega_{1}\left(b_{1}\right)=\omega_{0}\left(a_{1}\right)=\omega_{0}\left(\left(\omega_{0}+\omega_{1}\right)^{*}\right) .
$$

Следовательно,

$$
\begin{aligned}
\delta\left(\omega_{0}\right. & \left.+\omega_{1}+\omega_{2}\right)+\delta\left(\omega_{0}\right)+\delta\left(\omega_{1}\right)+\delta\left(\omega_{2}\right) \\
& =\left(\delta\left(\omega_{0}\right)+\delta\left(\omega_{0}+\omega_{1}+\omega_{2}\right)\right)+\left(\delta\left(\omega_{0}\right)+\delta\left(\omega_{1}\right)\right)+\left(\delta\left(\omega_{0}\right)+\delta\left(\omega_{2}\right)\right) \\
& =\omega_{0}\left(\left(\omega_{1}+\omega_{2}\right)^{*}\right)+\omega_{0}\left(\left(\omega_{0}+\omega_{1}\right)^{*}\right)+\omega_{0}\left(\left(\omega_{0}+\omega_{2}\right)^{*}\right) \\
& =\omega_{0}\left(\left(\omega_{0}+\omega_{1}\right)^{*}+\left(\omega_{0}+\omega_{2}\right)^{*}\right)+\omega_{0}\left(\left(\omega_{0}+\omega_{1}\right)^{*}\right)+\omega_{0}\left(\left(\omega_{0}+\omega_{2}\right)^{*}\right) \\
& =\left(\left(\omega_{0}+\omega_{1}\right)^{*},\left(\omega_{0}+\omega_{2}\right)^{*}\right) .
\end{aligned}
$$


ДоКАЗАТЕЛЬСТВо ТЕОРЕмы 9.1. В одну сторону утверждение теоремы очевидно. Пусть теперь наборы $\Omega^{1}$ и $\Omega^{2}$ такие, что

$$
\delta\left(\omega_{0}^{1}+\omega_{i}^{1}+\omega_{j}^{1}\right)=\delta\left(\omega_{0}^{2}+\omega_{1}^{2}+\omega_{2}^{2}\right)
$$

и, в частности, $\delta\left(\omega_{i}^{1}\right)=\delta\left(\omega_{i}^{2}\right)$ для всех $i, j=0,1, \ldots, n$.

Положим

$$
v_{i}^{j}=\left(\omega_{0}^{j}+\omega_{i}^{j}\right)^{*} \text { и } V^{j}=\left(v_{1}^{j}, \ldots, v_{n}^{j}\right) .
$$

Тогда согласно лемме 9.2

$$
\left(v_{i}^{1}, v_{j}^{1}\right)=\left(v_{i}^{2}, v_{j}^{2}\right) \text { и } \omega_{0}^{1}\left(v_{i}^{1}\right)=\omega_{0}^{2}\left(v_{i}^{2}\right) .
$$

Таким образом, согласно лемме 9.1 существует изоморфизм $\varphi: H \rightarrow H$, сохраняющий форму $(\cdot, \cdot)$, переводяший $V^{1}$ в $V^{2}$ и такой, что $\omega_{0}^{2} \varphi=\omega_{0}^{1}$. Используя скручивание Дена [39], легко показать, что сохраняюший форму $(\cdot, \cdot)$ автоморфизм $\varphi$ порождается гомеоморфизмом $\widetilde{\varphi}: P \rightarrow P$.

\section{$\S 10$. Пространство модулей спинорных расслоений ранга 1}

1. Пусть $e: E \rightarrow P$ - линейное локальное тривиальное голоморфное расслоение над римановой поверхностью $P$. Униформизация $\Lambda \rightarrow \Lambda / \Gamma$, где $\Gamma \subset P S L(2, \mathbb{R})$ - фуксова группа, переводит $e$ в расслоение $\widetilde{e}: \widetilde{E} \rightarrow \Lambda$. Оно допускает тривилизацию, т.е. биголоморфное отображение $\widetilde{\Phi}: \widetilde{E} \rightarrow \Lambda \times \mathbb{C}$, переводящее $\widetilde{e}$ в естественную проекцию $\widetilde{\lambda}:(\Lambda \times \mathbb{C}) \rightarrow \Lambda$. Таким образом, расслоение $e$ изоморфно расслоению, получающемуся факторизацией расслоения $(\Lambda \times \mathbb{C})$ по группе $\Gamma$, где элемент $\gamma \in \Gamma \subset P S L(2, \mathbb{R})$ действует на $(\Lambda \times \mathbb{C})$ по правилу:

$$
\gamma(z, x)=\left(\frac{a z+b}{c z+d}, f(\gamma, z) \cdot x\right)
$$

Функция $f: \Gamma \times \Lambda \rightarrow \mathbb{C}^{*}=\mathbb{C} \backslash 0$ называется функиией перехода.

В частности, если $E$ - кокасательное расслоение, то

$$
f(x, z)=\left(\frac{d \gamma}{d z}\right)^{-1}=(c z+d)^{2}
$$

Если функции перехода $f$ и $g$ отвечают расслоениям $E \rightarrow P$ и $F \rightarrow P$, то расслоению $E \otimes F \rightarrow P$ отвечает функция перехода $f \cdot g$.

Линейное расслоение $E \rightarrow P$ называется спинорнылм, если расслоение $E \otimes E \rightarrow P$ изоморфно кокасательному. Таким образом, его функция перехода имеет вид $f(\gamma, z)=$ $\sigma(a, b, c, d)(c z+d)$, где $\sigma^{2}(a, b, c, d)=1$. Сопоставим отображению $\gamma(z)=\frac{a z+b}{c z+d}$ из Г матрицу

$$
J_{e}^{*}(\gamma)=\sigma(a, b, c, d) \cdot\left(\begin{array}{ll}
a & b \\
c & d
\end{array}\right) \in S L(2, \mathbb{R})
$$

ЛЕмма 10.1. Отображсение $J_{e}^{*}: \Gamma \rightarrow S L(2, \mathbb{R})$ определено корректно и является мономорфизмом. 
ДокАЗАТЕльСтво. Корректность $J^{*}$ означает, что

$$
\sigma(-a,-b,-c,-d)=-\sigma(a, b, c, d) .
$$

Это в свою очередь сразу следует из определения $f$. Если

$$
\gamma_{1}(z)=\frac{a_{1} z+b_{1}}{c_{1} z+d_{1}}, \quad \gamma_{2}(z)=\frac{a_{2} z+b_{2}}{c_{2} z+d_{2}}
$$

то

$$
\gamma_{1} \gamma_{2}(z)=\frac{\widetilde{a} z+\widetilde{b}}{\widetilde{c} z+\widetilde{d}}, \text { где }\left(\begin{array}{cc}
\widetilde{a} & \widetilde{b} \\
\widetilde{c} & \widetilde{d}
\end{array}\right)=\left(\begin{array}{ll}
a_{1} & b_{1} \\
c_{1} & d_{2}
\end{array}\right)\left(\begin{array}{ll}
a_{2} & b_{2} \\
c_{2} & d_{2}
\end{array}\right) .
$$

Таким образом,

$$
\gamma_{1} \gamma_{2}(z, x)=\left(\frac{\widetilde{a} z+\widetilde{b}}{\widetilde{c} z+\widetilde{d}}, f\left(\gamma_{1} \gamma_{2}, z\right) x\right)=\left(\frac{\widetilde{a} z+\widetilde{b}}{\widetilde{c} z+\widetilde{d}}, \sigma(\widetilde{a}, \widetilde{b}, \widetilde{c}, \widetilde{d})(\widetilde{c} z+\widetilde{d})\right) .
$$

Но, с другой стороны,

$$
\begin{aligned}
\gamma_{1} \gamma_{2}(z, x) & =\gamma_{1}\left(\frac{a_{2} z+b_{2}}{c_{2} z+d_{2}}, f\left(\gamma_{2}, z\right) x\right) \\
& =\left(\frac{\widetilde{a} z+\widetilde{b}}{\widetilde{c} z+\widetilde{d}}, \sigma\left(a_{1}, b_{1}, c_{1}, d_{1}\right)\left(c_{1} \frac{a_{2} z+b_{2}}{c_{2} z+d_{2}}+d_{1}\right) \sigma\left(a_{2}, b_{2}, c_{2}, d_{2}\right)\left(c_{2} z+d_{2}\right) x\right) .
\end{aligned}
$$

Следовательно,

$$
\sigma(\widetilde{a}, \widetilde{b}, \widetilde{c}, \widetilde{d})(\widetilde{c} z+\widetilde{d})=\sigma\left(a_{1}, b_{1}, c_{1}, d_{1}\right) \sigma\left(a_{2}, b_{2}, c_{2}, d_{2}\right)(\widetilde{c} z+\widetilde{d})
$$

и

$$
\sigma(\widetilde{a}, \widetilde{b}, \widetilde{c}, \widetilde{d})\left(\begin{array}{cc}
\widetilde{a} & \widetilde{b} \\
\widetilde{c} & \widetilde{d}
\end{array}\right)=\sigma\left(a_{1}, b_{1}, c_{1}, d_{1}\right) \sigma\left(a_{2}, b_{2}, c_{2}, d_{2}\right)\left(\begin{array}{ll}
a_{1} & b_{1} \\
c_{1} & d_{1}
\end{array}\right)\left(\begin{array}{ll}
a_{2} & b_{2} \\
c_{2} & d_{2}
\end{array}\right) .
$$

Таким образом, $J_{e}^{*}$ - гомоморфизм. Если $J: S L(2, \mathbb{R}) \rightarrow P S L(2, \mathbb{R})$ - естественная проекция, то $J J_{e}^{*}=\mathrm{id}$ и, следовательно, $J_{e}^{*}$ - мономорфизм.

Согласно лемме $10.1 J_{e}^{*}(\Gamma)$ - поднятие групшы Г. Пусть $\omega_{e}: H_{1}\left(\Lambda / \Gamma, \mathbb{Z}_{2}\right) \rightarrow \mathbb{Z}_{2}-$ функция Арфа, отвечаюшая этому поднятию (см. §7).

Tеорема 10.1 [41], [43]. Соответствие е $\mapsto \omega_{е}$ устанавливает взаимно однозначное соответствие между спинорными расслоениями и функииями Арфана римановой поверхности $P=\Lambda / \Gamma$.

ДоказАТЕльство. Теорема 7.2 устанавливает взаимно однозначное соответствие между функциями Арфа на $P$ и поднятиями группы $Г$. Докажем, что отображение $e \mapsto J_{e}^{*}(\Gamma)$ устанавливает взаимно однозначное соответствие между спинорными расслоениями и поднятиями группы Г. Для этого достаточно сопоставить каждому поднятию $J^{*}: \Gamma \rightarrow S L(2, \mathbb{R})$ спинорное расслоение $e$ такое, что $J_{e}^{*}=J^{*}$. Такое расслоение определяется переходной функцией $f(\gamma, z)=c z+d$, где $J^{*}(\gamma)=\left(\begin{array}{ll}a & b \\ c & d\end{array}\right)$. 
ЗАмЕчАниЕ. В случае компактных поверхностей другое описание соответствия $e \mapsto \omega_{e}$ содержится в [46]-[48].

2. Рассмотрим теперь множество $S p$ всех пар $(P, e)$, где $e: E \rightarrow P$ - спинорное расслоение на $P$. Будем считать, что $\left(P^{\prime}, e^{\prime}\right)=(P, e)$, если существуют биголоморфные отображения $f_{E}: E \rightarrow E^{\prime}$ и $f_{P}: P \rightarrow P$ такие, что $e^{\prime} f_{E}=f_{P} e$. Согласно теореме 10.1 расслоение $(P, e)$ определяется парой $(P, \omega)$, где $\omega$ - функция Арфа на $P$. Таким образом, топология пространства модулей римановых поверхностей индуцирует топологию на $S p$. Пусть $\left(g, \delta, k_{\alpha}, m_{\alpha}\right)$ удовлетворяет условиям леммы 8.1. Обозначим через $S\left(g, \delta, k_{\alpha}, m_{\alpha}\right)$ множество всех спинорных расслоений $(P, e)$ таких, что $\omega_{e}-$ функция Арфа топологического типа $t=\left(g, \delta, k_{\alpha}, m_{\alpha}\right)$.

Tеорема 10.2 [43]. Пространство $S\left(g, \delta, k_{\alpha}, m_{\alpha}\right)$ - гомеоморфно $T_{t} / \operatorname{Mod}_{t}$, әде

$$
T_{t} \cong T_{g, k_{1}+k_{2}, m_{1}+m_{2}} \cong \mathbb{R}^{6 g+3\left(k_{1}+k_{2}\right)+2\left(m_{1}+m_{2}\right)-6}
$$

$u$

$$
\operatorname{Mod}_{t} \subset \operatorname{Mod}_{g, k_{1}+k_{2}, m_{1}+m_{2}}
$$

- подгруппа конечного индекса.

ДокАЗАТЕльство. Пусть $k=k_{1}+k_{2}, m=m_{1}+m_{2}, n=k+m+g$ и $\psi \in T_{g, k, m}$. По определению это означает, что $\psi \in \operatorname{Hom}(\gamma, \operatorname{Aut}(\Lambda))$, где группа $\gamma=\gamma_{g, n}$ порождена образуюшими $v_{g, n}=\left\{a_{i}, b_{i}(i=1, \ldots, g), c_{i}(i=g+1, \ldots, n)\right\}$. Пусть

$$
\widetilde{v}_{g, n}=\left\{\widetilde{a}_{i}, \widetilde{b}_{i}(i=1, \ldots, g), \widetilde{c}_{i}(i=g+1, \ldots, n)\right\}
$$

- отвечаюший ему базис $H_{1}\left(P, \mathbb{Z}_{2}\right)$, где $P=P_{\psi}=\Lambda / \psi(\gamma)$. Рассмотрим на $P$ функцию Арфа $\omega=\omega_{\psi}$ такую, что $\omega\left(a_{i}\right)=\omega\left(b_{i}\right)=0$ при $i<g, \omega\left(a_{g}\right)=\omega\left(b_{g}\right)=\delta, \omega\left(c_{j}\right)=0$ при $g+1 \leqslant j \leqslant g+k_{0}, g+k+1 \leqslant j \leqslant g+k+m_{1}$ и $\omega\left(c_{j}\right)=1$ в остальных случаях. Согласно теореме 10.1 функции Арфа $\omega_{\psi}$ отвечает спинорное расслоение $e_{\psi}: E_{\psi} \rightarrow P_{\psi}$. Соответствие $\psi \mapsto e_{\psi}$ определяет отображение

$$
\Psi: T_{g, k, m} \rightarrow S\left(g, \delta, k_{\alpha}, m_{\alpha}\right)
$$

Согласно лемме 8.1

$$
\Psi\left(T_{g, k, m}\right)=S\left(g, \delta, k_{\alpha}, m_{\alpha}\right)
$$

Прообраз каждой точки $s \in S\left(g, \delta, k_{\alpha}, m_{\alpha}\right)$ состоит из орбиты группы $\operatorname{Aut}\left(P_{\psi}\right)=$ $\operatorname{Mod}_{g, k, m}$, сохраняющей функцию Арфа $\omega_{\psi}$. Таким образом,

$$
S\left(g, \delta, k_{\alpha}, m_{\alpha}\right)=T_{g, k, m} / \operatorname{Mod}_{t}
$$

где $\operatorname{Mod}_{t}$ - дискретная группа. 


\section{$\S$ 11. Суперфуксовы группы, суперримановы поверхности и их топологические типы}

Пусть $L=L(\mathbb{K})$ - грассманова алгебра над полем $\mathbb{K}$ с бесконечным числом образующих $1, \ell_{1}, \ell_{2}, \ldots$ Каждьй элемент $a \in L(\mathbb{K})$ является конечной линейной комбинацией мономов $\ell_{i_{1}} \wedge \cdots \wedge \ell_{i_{n}}$ с коэффициентами из $\mathbb{K}$, т.е.

$$
a=a^{\#}+\sum a_{i} e_{i}+\sum_{i j} a_{i j} e_{i} \wedge e_{j}+\cdots
$$

Соответствие $a \mapsto a^{\#}$ определяет эпиморфизм \#: $L(\mathbb{K}) \rightarrow \mathbb{K}$.

Моном $\ell_{i_{1}} \wedge \cdots \wedge \ell_{i_{n}} \neq 0$ называется четным, если $n$ четно, и нечетны.м, если $n$ - нечетно. Константы также считаются четньми мономами. Линейные комбинации с коэффициентами из $\mathbb{K}$ четных (нечетных) мономов порождают линейное пространство $L_{0}(\mathbb{K})$ четных $\left(L_{1}(\mathbb{K})\right.$ нечетных $)$ элементов алгебры $L(\mathbb{K})$. Супераналог линейного пространства - это множество

$$
\mathbb{K}^{(n \mid m)}=\left\{\left(z_{1}, \ldots, z_{n} \mid \theta_{1}, \ldots, \theta_{m}\right): z_{i} \in L_{0}(\mathbb{K}), \theta_{j} \in L_{1}(\mathbb{K})\right\}
$$

В качестве поля $\mathbb{K}$ будем рассматривать поле комплексных чисел $\mathbb{C}$ или вешественных чисел $\mathbb{R}$.

Множество

$$
\Lambda^{N S}=\left\{\left(z \mid \theta_{1}, \ldots, \theta_{N}\right) \in C^{(1 \mid N)} \mid \operatorname{Im} z^{\#}>0\right\}
$$

назьвается верхней $N$-суперполуплоскостью. В этом параграфе мы будем иметь дело с 1-суперполуплоскостями $\Lambda^{S}=\Lambda^{1 S}$. Группа автоморфизмов $\operatorname{Aut}\left(\Lambda^{S}\right)$ суперобласти $\Lambda^{S}$ состоит из преобразований $A=A[a, b, c, d, \sigma \mid \varepsilon, \delta]$ вида

$$
A(z \mid \theta)=\left(\frac{a z+b}{c z+d}-\frac{(a d-b c)(\varepsilon+\delta z)}{(c z+d)^{2}} \theta, \frac{\sigma \sqrt{a d-b c}}{c z+d}\left(\theta+\varepsilon+\delta z+\frac{1}{2} \varepsilon \delta \theta\right)\right)
$$

где $a, b, c, d \in L_{0}(\mathbb{R}), \sigma= \pm 1, \varepsilon, \delta \in L_{1}(\mathbb{R}),(a d-b c)^{\#}>0$, а символом $\sqrt{\Delta}$ обозначается элемент из $L_{0}(\mathbb{R})$, однозначно определяемый свойствами $(\sqrt{\Delta})^{2}=\Delta$ и $(\sqrt{\Delta})^{\#}>0$ [2], [4].

Соответствие

$$
A \mapsto A^{\#}, \quad \text { где } A^{\#}(z)=\frac{a^{\#} z+b^{\#}}{c^{\#} z+d^{\#}},
$$

порождает эпиморфизм

$$
\text { \#: } \operatorname{Aut}\left(\Lambda^{S}\right) \rightarrow \operatorname{Aut}(\Lambda) .
$$

Элементы множества

$$
\operatorname{Aut}_{1}\left(\Lambda^{S}\right)=(\#)^{-1}\left(\operatorname{Aut}_{1}(\Lambda)\right)
$$

называются суперпараболическими, а элементы множества

$$
\operatorname{Aut}_{2}\left(\Lambda^{S}\right)=(\#)^{-1}\left(\operatorname{Aut}_{2}(\Lambda)\right)
$$

- супергиперболическими. 
Подгруппу $\Gamma \subset \operatorname{Aut}\left(\Lambda^{S}\right)$ мы называем суперфуксовой, если $\Gamma^{\#}=\#(\Gamma)-$ фуксова группа и \#: $\Gamma \rightarrow \Gamma^{\#}$ - изоморфизм. В этом параграфе мы будем рассматривать (не оговаривая это специально) лишш суперфуксовы групшы, состояшие из суперпараболических и супергиперболических автоморфизмов $\Lambda^{S}$.

Сопоставим автоморфизму $A=A[a, b, c, d, \sigma \mid \varepsilon, \delta]$ матрицу

$$
\bar{J}(A)=\frac{\sigma}{\sqrt{a^{\#} d^{\#}-c^{\#} d^{\#}}}\left(\begin{array}{ll}
a^{\#} & b^{\#} \\
c^{\#} & d^{\#}
\end{array}\right) \in S L(2, \mathbb{R}) .
$$

Лемма 11.1. Пусть Г - суперфуксова группа. Тогда соответствие $\bar{J}: \Gamma \rightarrow S L(2, \mathbb{R})$ является мономорфизмом $u$, следовательно, определяет поднятие $J^{*}: \Gamma^{\#} \rightarrow \bar{\Gamma}=\bar{J}(\Gamma)$.

ДокАЗАТЕльство. Прямая выкладка показьвает, что $\bar{J}$ - гомоморфизм. Кроме того, \# $=J \circ \bar{J}$ и мономорфность \# влечет мономорфность $\bar{J}$.

Пусть $\Gamma \subset \operatorname{Aut}\left(\Lambda^{S}\right)$ - суперфуксова группа. Фактор множества $\Lambda^{S} / \Gamma$ называется римановой суперповерхностью (или $N=1$ суперримановой поверхностью). Суперповерхности $P_{1}=\Lambda^{S} / \Gamma_{1}$ и $P_{2}=\Lambda^{S} / \Gamma_{2}$ считаются совпадаюшими, если $\Gamma_{1}$ и $\Gamma_{2}$ сопряжены в $\operatorname{Aut}\left(\Lambda^{S}\right)$. Проекции $\#: \Lambda^{S} \rightarrow \Lambda$ и \#: Г $\rightarrow \Gamma^{\#}$ определяют проекцию $\#: P \rightarrow P^{\#}=\Lambda / \Gamma^{\#}$.

Согласно теореме 7.2 поднятие $J^{*}$ задает функцию Арфа

$$
\omega_{P}: H_{1}\left(P^{\#}, \mathbb{Z}_{2}\right) \rightarrow \mathbb{Z}_{2}
$$

Топологический тип $\left(g, \delta, k_{\alpha}, m_{\alpha}\right)$ этой функции Арфа назовем топологическим типом суперповерхности $P$.

TеОрема 11.1. Пусть $Q$ - риманова поверхность $и \omega: H_{1}\left(Q, \mathbb{Z}_{2}\right) \rightarrow \mathbb{Z}_{2}-$ произвольная функиия Арфа. Тогда существует риманова суперповерхность $P$ такая, что $P^{\#}=Q u \omega_{P}=\omega$.

ДокаЗАТЕльство. Пусть $Q=\Lambda / \Gamma$, где $\Gamma$ - фуксова группа. Тогда согласно теореме 7.2 существует поднятие $J^{*}: \Gamma \rightarrow S L(2, \mathbb{R})$, порождающее функцию Арфа $\omega$. Сопоставим теперь автоморфизму $\gamma \in \Gamma \subset \operatorname{Aut}(\Lambda)$ автоморфизм $\gamma^{S} \in \Gamma \subset \operatorname{Aut}\left(\Lambda^{S}\right)$ вида

$$
\gamma^{S}(z \mid \theta)=\left(\frac{a z+b}{c z+d} \mid \frac{\theta}{c z+d}\right), \quad \text { где }\left(\begin{array}{ll}
a & b \\
c & d
\end{array}\right)=J^{*}(\gamma)
$$

Тогда множество

$$
\Gamma^{S}=\left\{\gamma^{S} \mid \gamma \in \Gamma\right\} \subset \operatorname{Aut}\left(\Lambda^{S}\right)
$$

является группой. Кроме того, $\left(\gamma^{S}\right)^{\#}=\gamma$ и, следовательно, $\Gamma^{S}-$ суперфуксова группа. Положим $P=\Lambda^{S} / \Gamma^{S}$. Тогда $P^{\#}=Q$ и $\omega_{P}=\omega$.

Согласно теореме 11.1 множество $T^{t}$ римановых суперповерхностей типа

$$
t=\left(g, \delta, k_{\alpha}, m_{\alpha}\right)
$$

непусто для каждого $t$, удовлетворяюшего условиям леммы 8.1. Наша ближайшая задача - описать множества $T^{t}$. 


\section{§ 12. Модули суперримановых поверхностей}

1. Простейшие супергиперболические и суперпараболические автоморфизмы имеют вид

$$
I_{\lambda}^{\sigma}(z \mid \theta)=(\lambda z \mid \sigma \sqrt{\lambda} \theta)
$$

и

$$
J_{\mu}^{\sigma}(z \mid \theta)=(z+\mu \mid \sigma \theta),
$$

где $\sigma= \pm 1, \lambda, \mu \in L_{0}(\mathbb{R}), \lambda^{\#}>1, \mu^{\#}>0$.

Прямой выкладкой нетрудно доказать, что всякий

$$
A \in \operatorname{Aut}_{2}\left(\Lambda^{S}\right)
$$

со свойством $A^{\#}(\infty) \neq \infty$ имеет вид

$$
A=I(\sigma, \lambda, \alpha, \beta \mid \varepsilon, \delta),
$$

где $\lambda, \alpha, \beta \in L_{0}(\mathbb{R}), \varepsilon, \delta \in L_{1}(\mathbb{R}), \sigma= \pm 1, \lambda^{\#}>1, \alpha^{\#} \neq \beta^{\#}$ и

$$
\begin{aligned}
A(z \mid \theta)=\left(\frac{(\lambda \alpha-\beta) z+(1-\lambda) \alpha \beta}{(\lambda-1) z+(\alpha-\lambda \beta)}-\frac{\lambda(\alpha-\beta)^{2}(\varepsilon+\delta z) \theta}{((\lambda-1) z+(\alpha-\lambda \beta))^{2}} \mid\right. \\
\left.\frac{\sigma \sqrt{\lambda}(\alpha-\beta)}{(\lambda-1) z+(\alpha-\lambda \beta)}\left(\theta+\varepsilon+\delta z+\frac{1}{2} \varepsilon \delta \theta\right)\right) .
\end{aligned}
$$

Положим

$$
I(\sigma, \lambda, \infty, 0 \mid 0,0)=I_{\lambda}^{\sigma} .
$$

Аналогично, всякий

$$
A \in \operatorname{Aut}_{1}\left(\Lambda^{S}\right)
$$

со свойством $A^{\#}(\infty) \neq \infty$ имеет вид

$$
A=J(\sigma, \lambda, \alpha \mid \varepsilon, \delta),
$$

где $\lambda, a \in L_{0}(\mathbb{R}), \varepsilon, \delta \in L_{1}(\mathbb{R}), \sigma= \pm 1, \lambda^{\#}>0$ и

$$
A(z \mid \theta)=\left(\frac{(1-\alpha \lambda) z+a^{2} \lambda}{-\lambda z+(1+a \lambda)}-\frac{(\varepsilon+\delta z) \theta}{(-\lambda z+(1+a z))^{2}} \mid \frac{\sigma\left(\theta+\varepsilon+\delta z+\frac{1}{2} \varepsilon \delta \theta\right)}{-\lambda z+(1+a \lambda)}\right) .
$$

Мы используем эту параметризацию множества $\operatorname{Aut}_{1}\left(\Lambda^{S}\right) \cup \mathrm{Aut}_{2}\left(\Lambda^{S}\right)$ для построения супераналога пространства Фрике-Клейна-Тайхмюллера.

Пусть, как и раньше, $\gamma_{g, n}$ - группа, порож денная образуюшими

$$
v_{g, n}=\left\{a_{i}, b_{i}(i=1, \ldots, g), c_{i}(i=g+1, \ldots, n)\right\}
$$

с определяющими соотношениями

$$
\prod_{i=1}^{g}\left[a_{i}, b_{i}\right] \prod_{i=g+1}^{n} c_{i}=1
$$

Положим $c_{i}=\left[a_{i}, b_{i}\right](i=1, \ldots, g)$.

Пусть $n=g+k+m, 6 g+3 k+2 m>6$ и $t=\left(g, \delta, k_{\alpha}, m_{\alpha}\right)-$ топологический тип некоторой функции Арфа на поверхности типа $(g, k, m)$. Согласно лемме 8.1 это означает, что

1) $k=k_{0}+k_{1}, m=m_{0}+m_{1}, k_{1}+m_{1} \equiv 0(\bmod 2) ;$

2) $\delta=0$ при $k_{1}+m_{1}>0$. 
Обозначим через $\widetilde{T}^{t}$ множество мономорфизмов $\psi: v_{g, n} \rightarrow \operatorname{Aut}\left(\Lambda^{S}\right)$ таких, что

1) $\left(\psi\left(v_{g, n}\right)\right)^{\#} \in \widetilde{T}_{g, k, m}$

2) суперриманова поверхность $P=\Lambda^{S} / \psi\left(\gamma_{g, n}\right)$ порождает на $P^{\#}$ функцию Арфа $\omega=\omega_{P}$ типа $t$;

3) естественная проекция $\left(\psi\left(\gamma_{g, n}\right)\right)^{\#} \rightarrow H_{1}\left(P^{\#}, \mathbb{Z}_{2}\right)$ переводит $v_{g, n}$ в стандартный базис $\left\{a_{i} b_{i}(i=1, \ldots, g), c_{i}(i=g+1, \ldots, n)\right\}$ такой, что $\omega\left(a_{i}\right)=\omega\left(b_{i}\right)=0$ для $i>1, \omega\left(a_{1}\right)=\omega\left(b_{1}\right)=\delta$ и $\omega\left(c_{i}\right)=0$, если и только если $g+1 \leqslant i \leqslant g+k_{0}$ или $g+k+1 \leqslant i \leqslant g+k+m_{0}$.

Соответствие $\psi \mapsto \psi^{\#}$, где $\psi^{\#}(d)=(\psi(d))^{\#}$, порождает отображение \#: $\widetilde{T}^{t} \rightarrow$ $\widetilde{T}_{g, k, m}$. Согласно теореме 11.1

$$
\#\left(\widetilde{T}^{t}\right)=\widetilde{T}_{g, k, m}
$$

Группа $\operatorname{Aut}\left(\Lambda^{S}\right)$ действует на $\widetilde{T}^{t}$ сопряжениями. Положим

$$
T^{t}=\widetilde{T}^{t} / \operatorname{Aut}\left(\Lambda^{S}\right)
$$

Для любой тройки точек

$$
\left(z_{1} \mid \theta_{1}\right),\left(z_{2} \mid \theta_{2}\right),\left(z_{3} \mid \theta_{3}\right) \in \mathbb{R}^{(1 \mid 1)}
$$

сушествует $h \in \operatorname{Aut}\left(\Lambda^{S}\right)$ такой, что

$$
h\left(z_{1} \mid \theta_{1}\right)=(\infty \mid 0), \quad h\left(z_{2} \mid \theta_{2}\right)=(0 \mid 0) \text { и } h\left(z_{3} \mid \theta_{3}\right)=(1 \mid \theta) .
$$

Кроме того, автоморфизмы, сохраняющие точки $(\infty \mid 0),(0 \mid 0)$ и переводящие точки вида $(1 \mid \theta)$ в точки такого же вида, совпадают с инволюцией $(z \mid \theta) \mapsto(z \mid-\theta)$. Это позволяет отождествить $T^{t}$ с множеством $\check{T}^{t} / \mathbb{Z}_{2}$, где

1) при $g=k=0$

$$
\check{T}^{t}=\left\{\psi \in \widetilde{T}^{t} \mid \psi\left(c_{1} c_{2}\right)=I_{\lambda}^{\sigma}, \psi\left(c_{1}\right)=J\left(\sigma_{1}, \lambda_{1},-1 \mid \varepsilon, \delta\right)\right\} ;
$$

2) при $g=0, k=1$

$$
\check{T}^{t}=\left\{\psi \in \widetilde{T}^{t} \mid \psi\left(c_{1}\right)=I_{\lambda_{1}}^{\sigma_{1}}, \psi\left(c_{2}\right)=J\left(\sigma_{2}, \lambda_{2}, 1 \mid \varepsilon, \delta\right)\right\} ;
$$

3) при $g=k=1$

$$
\check{T}^{t}=\left\{\psi \in \widetilde{T}^{t} \mid \psi\left(c_{1}\right)=I_{\lambda_{1}}^{\sigma_{1}}, \psi\left(b_{1}\right)=I\left(\sigma, \lambda_{2}, 1, \beta \mid \varepsilon, \delta\right)\right\}
$$

4) в остальных случаях

$$
\check{T}^{t}=\left\{\psi \in \widetilde{T}^{t} \mid \psi\left(c_{1}\right)=I_{\lambda_{1}}^{\sigma_{1}}, \psi\left(c_{2}\right)=I\left(\sigma_{2}, \lambda_{2}, 1, \beta \mid \varepsilon, \delta\right)\right\} .
$$


В этих обозначениях числа $\sigma, \sigma_{1}, \sigma_{2}$ однозначно определяются типом $t$. Опишем теперь отображение

$$
\Psi: \check{T}^{t} \rightarrow \mathbb{R}^{(6 g+3 k+2 m-6 \mid 4 g+2 k+2 m-4)} .
$$

Для этого представим элементы $\psi\left(v_{g, m}\right)$, где $\psi \in \check{T}^{t}$, в следуюшем виде:

$$
\begin{aligned}
\psi\left(c_{i}\right)= & I\left(\sigma_{i}, \lambda_{i}, \alpha_{i}, \beta_{i} \mid x_{i}, y_{i}\right) \quad \text { при } i \leqslant g+k, \\
\psi\left(c_{i}\right)= & J\left(\sigma_{i}, \lambda_{i}, a_{i} \mid x_{i}, y_{i}\right) \quad \text { при } i>g+k, \\
& \psi\left(a_{i}\right)=I\left(\sigma_{i}^{a}, \lambda_{i}^{a}, \delta_{i}, \eta_{i} \mid x_{i}^{a}, y_{i}^{a}\right), \\
& \psi\left(b_{i}\right)=I\left(\sigma_{i}^{b}, \lambda_{i}^{b}, \varepsilon_{i}, \xi_{i} \mid x_{i}^{b}, y_{i}^{b}\right) .
\end{aligned}
$$

При $g=k=0$ положим

$$
\begin{aligned}
\Psi(\psi)=\left\{\lambda_{1}, \lambda_{3}, \ldots, \lambda_{m-1}, a_{3}, \ldots, a_{m-2} \mid\right. \\
\left.x_{1}, y_{1}, x_{3}, y_{3}, \ldots, x_{m-1}, y_{m-1}\right\} \subset \mathbb{R}^{(2 m-6 \mid 2 m-4)} .
\end{aligned}
$$

При $g=0, k=1$ положим

$$
\Psi(\psi)=\left\{\lambda_{1}, \lambda_{2}, \ldots, \lambda_{m}, a_{3}, \ldots, a_{m-1} \mid x_{2}, y_{2}, \ldots, x_{m}, y_{m}\right\} \subset \mathbb{R}^{(2 m-3 \mid 2 m-2)} .
$$

При $g=k=1$ положим

$$
\begin{aligned}
\Psi(\psi)=\left\{\lambda_{1}, \ldots, \lambda_{m+1}, \eta_{1}, \varepsilon_{1}, \alpha_{2}, \beta_{2}, a_{3}, a_{4}, \ldots, a_{m} \mid\right. \\
\left.x_{2}, y_{2}, \ldots, x_{m}, y_{m}, x_{1}^{a}, y_{1}^{a}, x_{1}^{b}, y_{1}^{b}\right\} \subset \mathbb{R}^{(2 m+3 \mid 2 m+2)} .
\end{aligned}
$$

В остальных случаях:

при $m=0$ положим

$$
\begin{aligned}
\Psi(\psi)= & \left\{\lambda_{1}, \ldots, \lambda_{g+k-1}, \xi_{1}, \eta_{1}, \varepsilon_{1}, \ldots, \xi_{g}, \eta_{g}, \varepsilon_{g}, \beta_{2}, \alpha_{3}, \beta_{3}, \ldots, \alpha_{g+k-1}, \beta_{g+k-1} \mid\right. \\
& \left.x_{2}, y_{2}, \ldots, x_{g+k-1}, y_{g+k-1}, x_{1}^{a}, y_{1}^{a}, \ldots, x_{g}^{a}, y_{g}^{a}\right\} \subset \mathbb{R}^{(6 g+3 k-6 \mid 4 g+2 k-4)}, \\
\text { при } m= & 1 \\
\Psi(\psi)= & \left\{\lambda_{1}, \ldots, \lambda_{g+k}, \xi_{1}, \eta_{1}, \varepsilon_{1}, \ldots, \xi_{g}, \eta_{g}, \varepsilon_{g}, \beta_{2}, \alpha_{3}, \beta_{3}, \ldots, \alpha_{g+k-1}, \beta_{g+k-1},\right. \\
& \left.\alpha_{g+k} \mid x_{2}, y_{2}, \ldots, x_{g+k}, y_{g+k}, x_{1}^{a}, y_{1}^{a}, \ldots, x_{g}^{a}, y_{g}^{a}\right\} \subset \mathbb{R}^{(6 g+3 k-4 \mid 4 g+2 k-2)},
\end{aligned}
$$

а в остальных случаях

$$
\begin{gathered}
\Psi(\psi)=\left\{\lambda_{1}, \ldots, \lambda_{g+k+m-1}, \xi_{1}, \eta_{1}, \varepsilon_{1}, \ldots, \xi_{g}, \eta_{g}, \varepsilon_{g}, \beta_{2}, \alpha_{3}, \beta_{3}, \ldots,\right. \\
\alpha_{g+k}, \beta_{g+k}, a_{g+k+1}, \ldots, a_{g+k+m-2} \mid x_{2}, y_{2}, \\
\left.\ldots, x_{g+k+m-1}, y_{g+k+m-1}, x_{1}^{a}, y_{1}^{a}, \ldots, x_{g}^{a}, y_{g}^{a}\right\} \subset \mathbb{R}^{(6 g+3 k+2 m-6 \mid 4 g+2 k+2 m-4)} .
\end{gathered}
$$

Положим

$$
\widehat{T}^{t}=\Psi\left(\check{T}^{t}\right) .
$$

Будем говорить, что область $Q \subset \mathbb{R}^{(r \mid \ell)}$ сильно диффеоморфна $\mathbb{R}^{(r \mid \ell)}$, если $Q^{\#}$ диффеоморфна $\mathbb{R}^{r}$ и $Q=\#^{-1}(\#(Q))$.

Tеорема 12.1 [49]-[51]. Отображсение $\Psi: \check{T}^{t} \rightarrow \widehat{T}^{t}-$ взаимно однозначно и $\widehat{T}^{t}$ - сильно диффеоморфно

$$
\mathbb{R}^{(6 g+3 k+2 m-6 \mid 4 g+2 k+2 m-4)} .
$$


ДокАЗАТЕЛЬСтво. Тип $t$ однозначно определяет величины $\sigma_{i}, \sigma_{i}^{a}, \sigma_{i}^{b}$. Параметры $\lambda_{i}, \alpha_{i}, \beta_{i}, a_{i}, x_{i}, y_{i}$ однозначно определяют $\psi\left(c_{i}\right)$ при $i<n$, а следовательно, и

$$
\psi\left(c_{n}\right)=\left(\prod_{i=1}^{n-1} \psi\left(c_{i}\right)\right)^{-1}
$$

Это позволяет определить по формулам (3.6)-(3.9) параметры $\lambda_{i}^{a}, \lambda_{i}^{b}$ и $\delta_{i}$, которые вместе с параметрами $x_{i}^{a}, y_{i}^{a}, x_{i}, y_{i}$ однозначно определяют $\psi\left(a_{i}\right)$ и $\psi\left(b_{i}\right)$. Таким образом, отображение $\Psi$ - взаимно однозначно. Все ограничения на параметры сводятся к ограничениям на их числовые части. Таким образом,

$$
\widehat{T}^{t}=\#^{-1}\left(\#\left(\widehat{T}^{t}\right)\right) .
$$

Кроме того, согласно теореме 4.1

$$
\left(\widehat{T}^{t}\right)^{\#}=\widehat{T}_{g, k, m} \cong \mathbb{R}^{6 g+3 m+2 k-6} .
$$

Из нашей конструкции сразу следует

СлЕДСТвИЕ 12.1 [43], [50]. Пусть $t$ - топологический тип функции Арфа на поверхности типа $(g, k, m)$ и $M^{t}$ - множество всех суперримановых поверхностей mипа t. Тогда $M^{t}$ представляется в виде $T^{t} / \operatorname{Mod}^{t}$, где $T^{t}$ сильно диффеоморфно

$$
\mathbb{R}^{(6 g+3 k+2 m-6 \mid 4 g+2 k+2 m-4)} / \mathbb{Z}_{2},
$$

a группа $\operatorname{Mod}^{t}$ дискретно действует на $T^{t}$. Если $\omega-$ функция Арфа типа $t$, то

$$
\operatorname{Mod}^{t} \cong\left\{\alpha \in \operatorname{Mod}_{g, k, m} \mid \omega \alpha=\omega\right\}
$$

ПримеР. Рассмотрим пространство $M_{g}$ суперримановых поверхностей $P$ таких, что $P_{\#}^{\#}$ - компактная риманова поверхность рода $g>1$. На компактных поверхностях рода $g>0$ сушествуют функции Арфа ровно двух типов. Таким образом, $M_{g}$ распадается на 2 компоненты связности, каждая из которых диффеоморфна

$$
\mathbb{R}^{(6 g-6 \mid 4 g-4)} / \operatorname{Mod},
$$

где Mod - зависящая от компоненты дискретная группа. 
$\S$ 13. $N=2$ суперфуксовы группы. $N=2$ суперримановы поверхности и их топологические инварианты

Обозначим через $A[a, b, c, d, \ell \mid \varepsilon]$ отображение $A: \Lambda^{2 S} \rightarrow \Lambda^{2 S}$ вида

$$
\begin{aligned}
& A\left(z \mid \theta_{1}, \theta_{2}\right)=\left(\frac{a z+b+\delta^{11} \theta_{1}+\delta^{12} \theta_{2}}{c z+d+\delta^{21} \theta_{1}+\delta^{22} \theta_{2}} \mid\right. \\
&\left.\frac{\ell^{11} \theta_{1}+\ell^{12} \theta_{2}+\varepsilon^{11} z+\varepsilon^{12}}{c z+d+\delta^{21} \theta_{1}+\delta^{22} \theta_{2}}, \frac{\ell^{21} \theta_{1}+\ell^{22} \theta_{2}+\varepsilon^{21} z+\varepsilon^{22}}{c z+d+\delta^{21} \theta_{1}+\delta^{22} \theta_{2}}\right),
\end{aligned}
$$

где $a, b, c, d \in L_{0}(\mathbb{R}), \ell \in G L\left(2, L_{0}(\mathbb{R})\right), \varepsilon^{i j}, \delta^{i j} \in L_{1}(\mathbb{R})$.

Согласно [6] группа автоморфизмов $\operatorname{Aut}\left(\Lambda^{2 S}\right)$ суперобласти $\Lambda^{2 S}$ состоит из $A[a, b, c, d, \ell \mid \varepsilon]$, где

$$
\left(\begin{array}{ll}
-c & a \\
-d & b
\end{array}\right)\left(\begin{array}{ll}
\delta^{11} & \delta^{12} \\
\delta^{21} & \delta^{22}
\end{array}\right)=\left(\begin{array}{ll}
\varepsilon^{21} & \varepsilon^{11} \\
\varepsilon^{22} & \varepsilon^{12}
\end{array}\right)\left(\begin{array}{ll}
\ell^{11} & \ell^{12} \\
\ell^{21} & \ell^{22}
\end{array}\right)
$$

и

$$
a d-b c-\varepsilon^{11} \varepsilon^{12}-\varepsilon^{21} \varepsilon^{22}=\ell^{11} \ell^{22}+\ell^{21} \ell^{12}+\delta^{11} \delta^{22}+\delta^{12} \delta^{21}=\Delta,
$$

где $\Delta^{\#}>0$

$$
\ell^{11} \ell^{21}+\delta^{11} \delta^{21}=\ell^{12} \ell^{22}+\delta^{12} \delta^{22}=0
$$

Прямой выкладкой нетрудно показать, что верны следующие леммы.

ЛЕмма 13.1. Автоморфизм $A[a, b, c, d, \ell \mid \varepsilon]$ относится $к$ одному из двух тиnов:

1) (нескрученных $)\left(\ell^{12}\right)^{\#}=\left(\ell^{21}\right)^{\#}=0$;

2) $($ скрученных $)\left(\ell^{11}\right)^{\#}=\left(\ell^{22}\right)^{\#}=0$.

Нескрученный (скрученный) автоморфизм однозначно определяется параметрами $a, b, c, d, \varepsilon^{i j}, \ell^{11}$ (соответственно $a, b, c, d, \varepsilon^{i j}, \ell^{12}$ ). Эти параметры могут принимать произвольные значения такие, что $a, b, c, d, \ell^{i j} \in L_{0}(\mathbb{R}), \varepsilon^{i j} \in L_{1}(\mathbb{R})$, $(a d-b c)^{\#}>0,\left(\ell^{11}+\ell^{12}\right)^{\#} \neq 0$.

Лемма 13.2. Пусть $\left(z_{i} \mid \theta_{i}^{1}, \theta_{i}^{2}\right) \in \mathbb{R}^{(1 \mid 2)}, i=1,2,3, u z_{i}^{\#} \neq z_{j}^{\#}$ при $i \neq j$. Тогда существует $A \in \operatorname{Aut}\left(\Lambda^{2 S}\right)$ такой, что $A\left(z_{1} \mid \theta_{1}^{1}, \theta_{1}^{2}\right)=(0 \mid 0,0), A\left(z_{2} \mid \theta_{2}^{1}, \theta_{2}^{2}\right)=$ $(\infty \mid 0,0)$ u $A\left(z_{3} \mid \theta_{3}^{1}, \theta_{3}^{2}\right)=\left(1 \mid \theta^{1}, \theta^{2}\right)$.

Положим

$$
\begin{aligned}
\operatorname{Aut}_{0}^{2 t}=\left\{h \in \operatorname{Aut}\left(\Lambda^{2 S}\right) \mid\right. & \\
& \left.\quad h(0 \mid 0,0)=(0 \mid 0,0), h(\infty \mid 0,0)=(\infty \mid 0,0), h(1 \mid 0,0)=\left(1 \mid \theta^{1}, \theta^{2}\right)\right\} .
\end{aligned}
$$

Лемма 13.3. Группа $A_{0}^{2 S}$ состоит из әлементов вида $h\left(z \mid \theta^{1}, \theta^{2}\right)=\left(z \mid \mu \theta^{i}\right.$, $\left.\mu^{-1} \theta^{3-i}\right)$, где $\mu \in L_{0}(\mathbb{R}), \mu^{\#} \neq 0$ и і равно 1 или 2. 
ДокАЗАТЕльство. Определение $A_{0}^{2 S}$ влечет $h=A[1,0,0,1, \ell \mid 0]$, откуда $\ell^{11} \ell^{21}=$ $\ell^{12} \ell^{22}=0, \ell^{11} \ell^{22}+\ell^{12} \ell^{21}=1$. Следовательно, или $\ell^{12}=\ell^{21}=0, \ell^{11} \ell^{22}=1$, или $\ell^{11}=\ell^{22}=0, \ell^{12} \ell^{21}=1$.

Соответствие $A \mapsto A^{\#}$, где

$$
A=A[a, b, c, d, \ell \mid \varepsilon], \quad A^{\#}(z)=\frac{a^{\#} z+b^{\#}}{c^{\#} z+d^{\#}}
$$

порождает эпиморфизм \#: $\operatorname{Aut}\left(\Lambda^{2 S}\right) \rightarrow \operatorname{Aut}(\Lambda)$. Элемент множества $\operatorname{Aut}_{1}\left(\Lambda^{2 S}\right)=$ $(\#)^{-1}\left(\operatorname{Aut}_{1}(\Lambda)\right)$ назьвается суперпараболическим, а элемент множества $\operatorname{Aut}_{2}\left(\Lambda^{2 S}\right)$ $=(\#)^{-1}\left(\operatorname{Aut}_{2}(\Lambda)\right)-$ супергиперболическим.

Подгруппа $\Gamma \subset \operatorname{Aut}\left(\Lambda^{2 S}\right)$ называется $N=2$ суперфуксовой, если $\Gamma^{\#}=\#(\Gamma)-$ фуксова группа и \# : $\rightarrow \Gamma^{\#}-$ изоморфизм. В этом параграфе мы будем рассматривать (не оговаривая это специально) лишш $N=2$ суперфуксовы групшы, состояшие из суперпараболических и супергиперболических автоморфизмов $\Lambda^{2 S}$.

Из соотношений на $\ell^{i j}$ следует, что $\left(\ell^{11} \ell^{22}\right)^{\#} \geqslant 0,\left(\ell^{12} \ell^{21}\right)^{\#} \geqslant 0$ и или $\left(\ell^{11}\right)^{\#}=$ $\left(\ell^{22}\right)^{\#}=0$, или $\left(\ell^{12}\right)^{\#}=\left(\ell^{21}\right)^{\#}=0$.

Сопоставим автоморфизму $A=A[a, b, c, d, \ell \mid \varepsilon]$ матрицу

$$
\bar{J}(A)=\frac{\sigma}{\sqrt{a^{\#} d^{\#}-b^{\#} c^{\#}}}\left(\begin{array}{cc}
a^{\#} & b^{\#} \\
c^{\#} & d^{\#}
\end{array}\right) \in S L(2, \mathbb{R}),
$$

где $\sigma=\operatorname{sign}\left(\ell^{11}+\ell^{12}+\ell^{21}+\ell^{22}\right)^{\#}$.

Лемма 13.4. Пусть $\Gamma \in \operatorname{Aut}\left(\Lambda^{2 S}\right)$ есть $N=2$ суперфуксова группа. Тогда соответствие $\bar{J}: \Gamma \rightarrow S L(2, \mathbb{R})$ является мономорфизмом и, следовательно, определяет поднятие $J^{*}: \Gamma^{\#} \rightarrow \bar{\Gamma}=\bar{J}(\Gamma)$.

ДокАЗАТЕльство. Прямая выкладка показьвает, что $\bar{J}$ - гомоморфизм. Кроме того, \# $=J \circ \bar{J}$ и мономорфность \# влечет мономорфность $\bar{J}$.

Пусть $\Gamma \subset \operatorname{Aut}\left(\Lambda^{2 S}\right)$ есть $N=2$ суперфуксова группа. Фактормножество $\Lambda^{2 S} / \Gamma$ назьвается римановой $N=2$ суперповерхностью или $N=2$ суперримановой поверхностью. Две $N=2$ суперповерхности $P_{1}=\Lambda^{2 S} / \Gamma_{1}$ и $P_{2}=\Lambda^{2 S} / \Gamma_{2}$ считаются совпадающими, если $\Gamma_{1}$ и $\Gamma_{2}$ сопряжены в $\operatorname{Aut}\left(\Lambda^{2 S}\right)$. Проекции $\#: \Lambda^{2 S} \rightarrow \Lambda$ и $\#: \Gamma \rightarrow \Gamma^{\#}$ определяют проекцию \# $P \rightarrow P^{\#}=\Lambda / \Gamma^{\#}$.

Согласно теореме 7.2 поднятие $J^{*}$ задает функцию Арфа

$$
\omega_{P}^{1}: H_{1}\left(P^{\#}, \mathbb{Z}_{2}\right) \rightarrow \mathbb{Z}_{2}
$$

Рассмотрим теперь функцию

$$
\Omega: \Gamma \rightarrow \mathbb{Z}_{2}=\{0,1\}
$$

сопоставляюшую автоморфизму $A[a, b, c, d, \ell \mid \varepsilon] \in \Gamma$ значение 0 , если $\left(\ell^{12}\right)^{\#}=$ $\left(\ell^{21}\right)^{\#}=0$, и значение 1 , если $\left(\ell^{11}\right)^{\#}=\left(\ell^{22}\right)^{\#}=0$. Нетрудно видеть, что $\Omega-$ гомоморфизм, индуцирующий гомоморфизм $\omega_{P}^{0}: H_{1}\left(P^{\#}, \mathbb{Z}_{2}\right) \rightarrow \mathbb{Z}_{2}$. Обозначим через $\omega_{P}^{2}$ функцию Арфа $\omega_{P}^{1}+\omega_{P}^{0}$. 
Tеорема 13.1. Пусть $Q$ - риманова поверхность $и \omega^{1}, \omega^{2}: H_{1}\left(Q, \mathbb{Z}_{2}\right) \rightarrow \mathbb{Z}_{2}-$ произвольные функиии Арфа. Тогда существует риманова $N=2$ суперповерхность $P$ такая, ито $P^{\#}=Q u \omega_{P}^{1}=\omega^{1}, \omega_{P}^{2}=\omega^{2}$.

ДокАЗАТЕЛЬСтво. Пусть $Q=\Lambda / \Gamma$, где $\Gamma$ - фуксова группа. Тогда согласно теореме 7.2 сушествует поднятие $J^{*}: \Gamma \rightarrow S L(2, \mathbb{R})$, порождающее функции Арфа $\omega^{1}$. Сопоставим теперь автоморфизму $\gamma \in \Gamma \subset \operatorname{Aut}(\Lambda)$ автоморфизм $\gamma^{S} \in \operatorname{Aut}\left(\Lambda^{2 S}\right)$ вида

$$
\gamma^{S}\left(z \mid \theta_{1}, \theta_{2}\right)=\left(\frac{a z+b}{c z+d} \mid \frac{\theta_{i}}{c z+d}, \frac{\theta_{3-i}}{c z+d}\right),
$$

где $\left(\begin{array}{ll}a & b \\ c & d\end{array}\right)=J^{*}(\gamma)$ и

$$
i= \begin{cases}1, & \text { если } \omega^{1}(\gamma)=\omega^{2}(\gamma), \\ 2, & \text { если } \omega^{1}(\gamma) \neq \omega^{2}(\gamma) .\end{cases}
$$

Так как $\omega^{1}+\omega^{2}$ - гомоморфизм, то $\Gamma^{S}=\left\{\gamma^{S} \mid \gamma \in \Gamma\right\}$ есть $N=2$ суперфуксова группа и $P=\Lambda^{2 S} / \Gamma^{S}$ есть риманова $N=2$ суперповерхность. При этом $P^{\#}=Q$, $\omega_{P}^{1}=\omega^{1}$ и $\omega_{P}^{2}=\omega^{2}$.

Риманову $N=2$ суперповерхность $P$ назовем нескрученной, если $\omega_{P}^{0}=0$. Ее топологическим типом назовем топологический тип $\left(g, \delta, k_{\alpha}, m_{\alpha}\right)$ функции Арфа $\omega_{P}^{1}=\omega_{P}^{2}$. Если $\omega_{P}^{0} \neq 0$, то риманову поверхность назовем скрученной. Ее топологическим типом назовем топологический тип $\left(g, \delta_{1}, \delta_{2}, k_{\alpha \beta}, m_{\alpha \beta}\right)$ пары функций Арфа $\left(\omega_{P}^{1}, \omega_{P}^{2}\right)$.

Наша ближайшая задача - описать множества $M^{2 t}$ римановых $N=2$ суперповерхностей произвольного топологического типа $t$.

\section{$\S$ 14. Модули $N=2$ суперримановых поверхностей}

Простейшие супергиперболические и суперпараболические автоморфизмы суперобласти $\Lambda^{2 S}$ имеют вид

$$
I_{\lambda}^{\ell}\left(z \mid \theta_{1}, \theta_{2}\right)=\left(\lambda z \mid \ell^{11} \theta_{1}+\ell^{12} \theta_{2}, \ell^{21} \theta_{1}+\ell^{22} \theta_{2}\right)
$$

и

$$
J_{\mu}^{\ell}\left(z \mid \theta_{1}, \theta_{2}\right)=\left(z+\mu \mid \ell^{11} \theta_{1}+\ell^{12} \theta_{2}, \ell^{21} \theta_{1}+\ell^{22} \theta_{2}\right),
$$

где $\lambda, \mu \in L_{0}(\mathbb{R}), \lambda^{\#}>1, \mu^{\#}>0, \ell \in G L\left(2, L_{0}(\mathbb{R})\right)$ и $\ell^{11} \ell^{21}=\ell^{12} \ell^{22}=0, \ell^{11} \ell^{22}+$ $\ell^{12} \ell^{21}$ равна $\lambda$ для $I_{\lambda}^{\ell}$ и 1 для $J_{\mu}^{\ell}$.

Всякий $A \in \operatorname{Aut}_{2}\left(\Lambda^{2 S}\right)$ со свойством $A^{\#}(\infty) \neq \infty$ имеет вид

$$
A=I(\ell, \lambda, \alpha, \beta \mid \varepsilon)=A[\lambda \alpha-\beta,(1-\alpha) \alpha \beta, \lambda-1, \alpha-\lambda \beta, \ell \mid \varepsilon],
$$

где $\lambda, \alpha, \beta \in L_{0}(\mathbb{R}), \lambda^{\#}>1, \alpha^{\#} \neq \beta^{\#}, \ell \in G L\left(2, L_{0}(\mathbb{R})\right), \varepsilon \in G L\left(2, L_{1}(\mathbb{R})\right)$. Положим

$$
I(\ell, \lambda, \infty, 0 \mid 0)=I_{\lambda}^{\ell} .
$$


Всякий $A \in \operatorname{Aut}_{1}\left(\Lambda^{2 S}\right)$ со свойством $A^{\#}(\infty) \neq \infty$ имеет вид

$$
A=J(\ell, \lambda, a \mid \varepsilon)=A\left[1-a \lambda, a^{2} \lambda,-\lambda, 1+a \lambda, \ell \mid \varepsilon\right],
$$

где $\lambda, a \in L_{0}(\mathbb{R}), \lambda^{\#}>0, \ell \in G L\left(2, L_{0}(\mathbb{R})\right), \varepsilon \in G L\left(2, L_{1}(\mathbb{R})\right)$.

Как и в случае $N=1$ суперповерхностей, мы используем эту параметризацию множества $\operatorname{Aut}_{1}\left(\Lambda^{2 S}\right) \cup \operatorname{Aut}_{2}\left(\Lambda^{2 S}\right)$ для построения $N=2$ супераналога пространства Фрике-Клейна-Тайхмюллера.

Обозначим через $\widetilde{T}^{2 t}-$ множество мономорфизмов $\psi: v_{g, n} \rightarrow \operatorname{Aut}\left(\Lambda^{2 S}\right)$ таких, что:

1) $\left(\psi\left(v_{g, n}\right)\right) \# \in \widetilde{T}_{g, k, m}$

2) $P=\Lambda^{2 S} / \psi\left(\gamma_{g, n}\right)$ - риманова $N=2$ суперповерхность типа $t$;

$3)$ естественная проекция $\left(\psi\left(\gamma_{g, n}\right)\right)^{\#} \rightarrow H_{1}\left(P^{\#}, \mathbb{Z}_{2}\right)$ переводит $v_{g, n}$ в стандартный базис $\left\{a_{i}, b_{i}(i=1, \ldots, g), c_{i}(i=g+1, \ldots, n)\right\}$ такой, что $\omega_{i}=\omega_{P}^{i}$ удовлетворяют условиям леммы 8.1 (если суперповерхность нескручена) или условиям леммы 8.2 (если суперповерхность скручена).

Соответствие $\psi \mapsto \psi^{\#}$, где $\psi^{\#}(d)=(\psi(d))^{\#}$, порождает отображение \#: $\widetilde{T}^{2 t} \rightarrow$ $\widetilde{T}_{g, k, m}$. Согласно теореме $13.1 \#\left(\widetilde{T}^{2 t}\right)=\widetilde{T}_{g, k, m}$.

Соответствие $\Psi: \psi \mapsto \Lambda^{2 S} / \psi\left(\gamma_{g, n}\right)$ переносит топологию с пространства $\widetilde{T}^{2 t}$ на пространство $M_{2 t} N=2$ суперримановых поверхностей типа $t$.

Рассмотрим теперь пространство $\check{T}^{2 t}$, определенное условиями

1) при $g=k=0$

$$
\check{T}^{2 t}=\left\{\psi \in \widetilde{T}^{2 t} \mid \psi\left(c_{1} c_{2}\right)=I_{\lambda}^{\ell}, \psi\left(c_{1}\right)=J\left(\ell_{1}, \lambda_{1},-1, \mid \varepsilon\right)\right\} ;
$$

2) при $g=0, k=1$

$$
\check{T}^{2 t}=\left\{\psi \in \widetilde{T}^{2 t} \mid \psi\left(c_{1}\right)=I_{\lambda_{1}}^{\ell_{1}}, \psi\left(c_{2}\right)=J\left(\ell_{2}, \lambda_{2}, 1, \mid \varepsilon\right)\right\} ;
$$

3) при $g=k=1$

$$
\check{T}^{2 t}=\left\{\psi \in \widetilde{T}^{2 t} \mid \psi\left(c_{1}\right)=I_{\lambda_{1}}^{\ell_{1}}, \psi\left(b_{1}\right)=I\left(\ell, \lambda_{2}, 1, \mid \varepsilon\right)\right\} ;
$$

4) в остальных случаях

$$
\check{T}^{2 t}=\left\{\psi \in \widetilde{T}^{2 t} \mid \psi\left(c_{1}\right)=I_{\lambda_{1}}^{\ell_{1}}, \psi\left(c_{2}\right)=I\left(\ell_{2}, \lambda_{2}, 1, \beta, \mid \varepsilon\right)\right\} .
$$

Опишем теперь отображение

$$
\Psi^{2 t}: \check{T}^{2 t} \rightarrow \mathbb{R}^{\left(r_{1} \mid r_{2}\right)}
$$

Для этого представим элементы $\psi\left(v_{g, m}\right)$, где $\psi \in T^{2 t}$, в следуюшем виде:

$$
\begin{aligned}
\psi\left(c_{i}\right)= & I\left(\ell_{i}, \lambda_{i}, \alpha_{i}, \beta_{i} \mid z_{i}\right) \text { при } i \leqslant g+k, \\
\psi\left(c_{i}\right)= & J\left(\ell_{i}, \lambda_{i}, a_{i} \mid z_{i}\right) \quad \text { при } i>g+k, \\
& \psi\left(a_{i}\right)=I\left(\ell_{i}^{a}, \lambda_{i}^{a}, \delta_{i}, \eta_{i} \mid z_{i}^{a}\right), \\
& \psi\left(b_{i}\right)=I\left(\ell_{i}^{b}, \lambda_{i}^{b}, \varepsilon_{i}, \xi_{i} \mid z_{i}^{b}\right) .
\end{aligned}
$$


Согласно лемме 13.1 при заданном топологическом типе $t$ матрицы $\ell, \ell_{i}, \ell_{i}^{a}, \ell_{i}^{b}$ определяются величинами

$$
\tilde{\ell}, \tilde{\ell}_{i}, \tilde{\ell}_{i}^{a}, \tilde{\ell}_{i}^{b} \in \Lambda_{0}(\mathbb{R})
$$

где

$$
\tilde{\ell}, \tilde{\ell}_{i}, \tilde{\ell}_{i}^{a}, \tilde{\ell}_{i}^{b}=\left( \pm \ell, \pm \ell_{i}, \pm \ell_{i}^{a}, \pm \ell_{i}^{b}\right),
$$

с положительной числовой частью $\tilde{\ell}^{\#}, \tilde{\ell}_{i}^{\#},\left(\tilde{\ell}_{i}^{a}\right)^{\#},\left(\tilde{\ell}_{i}^{b}\right) \#>0$.

Для нескрученного топологического типа $t$ при $k=m=0$ положим

$$
\begin{aligned}
\Psi(\psi)=\left\{\lambda_{1}, \ldots, \lambda_{g-1}, \tilde{\ell}_{1}^{a}, \tilde{\ell}_{1}^{b}, \ldots, \tilde{\ell}_{g}^{a}, \tilde{\ell}_{g}^{b}, \xi_{1}, \eta_{1}, \varepsilon_{1}, \ldots, \xi_{g}, \eta_{g}, \varepsilon_{g},\right. \\
\left.\quad \beta_{2}, \alpha_{3}, \beta_{3}, \ldots, \alpha_{g-1}, \beta_{g-1} \mid z_{2}, \ldots, z_{g-1}, z_{1}^{a}, \ldots, z_{g}^{a}\right\} \subset \mathbb{R}^{(8 g-6 \mid 8 g-8)} .
\end{aligned}
$$

Для скрученного топологического типа $t$ при $k=m=0$ положим

$$
\begin{aligned}
\Psi(\psi)=\left\{\lambda_{1}, \ldots, \lambda_{g-1}, \tilde{\ell}_{1}^{b}, \tilde{\ell}_{2}^{a}, \tilde{\ell}_{2}^{b}, \ldots, \tilde{\ell}_{g}^{a}, \tilde{\ell}_{g}^{b}, \xi_{1}, \eta_{1}, \varepsilon_{1}, \ldots, \xi_{g}, \eta_{g}, \varepsilon_{g},\right. \\
\\
\left.\quad \beta_{2}, \alpha_{3}, \beta_{3}, \ldots, \alpha_{g-1}, \beta_{g-1} \mid z_{2}, \ldots, z_{g-1}, z_{1}^{a}, \ldots, z_{g}^{a}\right\} \subset \mathbb{R}^{(8 g-7 \mid 8 g-8)} .
\end{aligned}
$$

При $g=k=0$ положим

$$
\begin{aligned}
& \Psi(\psi)=\left\{\tilde{\ell}, \tilde{\ell}_{1}, \tilde{\ell}_{3}, \ldots, \tilde{\ell}_{m-1}, \lambda_{1}, \lambda_{3}, \ldots, \lambda_{m-1}, a_{3}, \ldots, a_{m-2} \mid\right. \\
& \left.z_{1}, z_{3}, \ldots, z_{m-1}\right\} \subset \mathbb{R}^{(3 m-7 \mid 4 m-8)} \text {. }
\end{aligned}
$$

При $g=0, k=1$ положим

$$
\begin{array}{r}
\Psi(\psi)=\left\{\tilde{\ell}_{1}, \tilde{\ell}_{2}, \ldots, \tilde{\ell}_{m}, \lambda_{1}, \lambda_{2}, \ldots, \lambda_{m}, a_{3}, \ldots, a_{m-1} \mid\right. \\
\left.z_{2}, \ldots, z_{m}\right\} \subset \mathbb{R}^{(3 m-3 \mid 4 m-4)} .
\end{array}
$$

При $g=k=1$ положим

$$
\begin{aligned}
\Psi(\psi)=\left\{\tilde{\ell}_{2}, \ldots, \tilde{\ell}_{m+1}, \lambda_{1}, \ldots, \lambda_{m+1}, \tilde{\ell}_{1}^{a}, \tilde{\ell}_{1}^{b}, \eta_{1}, \varepsilon_{1}, \alpha_{2}, \beta_{2}, a_{3}, \ldots, a_{m} \mid\right. \\
\left.z_{2}, \ldots, z_{m}, z_{1}^{a}, z_{1}^{b}\right\} \subset \mathbb{R}^{(3 m+5 \mid 4 m+4) .} .
\end{aligned}
$$

В остальных случаях:

при $m=0$ положим

$$
\begin{array}{r}
\Psi(\psi)=\left\{\tilde{\ell}_{g+1}, \ldots, \tilde{\ell}_{g+k-1}, \lambda_{1}, \ldots, \lambda_{g+k-1}, \tilde{\ell}_{1}^{a}, \tilde{\ell}_{1}^{b}, \ldots, \tilde{\ell}_{g}^{a}, \tilde{\ell}_{g}^{b}\right. \\
\xi_{1}, \eta_{1}, \varepsilon_{1}, \ldots, \xi_{g}, \eta_{g}, \varepsilon_{g}, \beta_{2}, \alpha_{3}, \beta_{3}, \ldots, \alpha_{g+k-1}, \beta_{g+k-1} \mid \\
\left.z_{2}, \ldots, z_{g+k-1}, z_{1}^{a}, \ldots, z_{g}^{a}\right\} \subset \mathbb{R}^{(8 g+4 k-7 \mid 8 g+4 k-8)},
\end{array}
$$

при $m=1$ положим

$$
\begin{aligned}
\Psi(\psi)=\left\{\tilde{\ell}_{g+1}, \ldots, \tilde{\ell}_{g+k}, \lambda_{1}, \ldots, \lambda_{g+k}, \tilde{\ell}_{1}^{a}, \tilde{\ell}_{1}^{b}, \ldots, \tilde{\ell}_{g}^{a}, \tilde{\ell}_{g}^{b}\right. \\
\xi_{1}, \eta_{1}, \varepsilon_{1}, \ldots, \xi_{g}, \eta_{g}, \varepsilon_{g}, \beta_{2}, \alpha_{3}, \beta_{3}, \ldots, \alpha_{g+k-1}, \beta_{g+k-1}, \alpha_{g+k} \mid \\
\left.z_{2}, \ldots, z_{g+k}, z_{1}^{a}, \ldots, z_{g}^{a}\right\} \subset \mathbb{R}^{(8 g+4 k-4 \mid 8 g+4 k-4)},
\end{aligned}
$$


а в остальных случаях

$$
\begin{gathered}
\Psi(\psi)=\left\{\tilde{\ell}_{g+1}, \ldots, \tilde{\ell}_{g+k+m-1}, \lambda_{1}, \ldots, \lambda_{g+k+m-1}, \tilde{\ell}_{1}^{a}, \tilde{\ell}_{1}^{b}, \ldots, \tilde{\ell}_{g}^{a}, \tilde{\ell}_{g}^{b}\right. \\
\xi_{1}, \eta_{1}, \varepsilon_{1}, \ldots, \xi_{g}, \eta_{g}, \varepsilon_{g}, \beta_{2}, \alpha_{3}, \beta_{3}, \ldots, \alpha_{g+k}, \beta_{g+k}, a_{g+k+1}, \ldots, a_{g+k+m-2} \mid \\
\left.z_{2}, \ldots, z_{g+k+m-1}, z_{1}^{a}, \ldots, z_{g}^{a}\right\} \subset \mathbb{R}^{(8 g+4 k+3 m-7 \mid 8 g+4 k+4 m-8)} .
\end{gathered}
$$

Положим

$$
\widehat{T}^{2 t}=\Psi\left(\check{T}^{2 t}\right) \text {. }
$$

Теорема 14.1. Отображение $\Psi: \check{T}^{2 t} \rightarrow \widehat{T}^{2 t}$ - взаимно однозначно и $\widehat{T}^{2 t}$ сильно диффеоморфно

$$
\mathbb{R}^{(8 g+4 k+3 m-b \mid 8 g+4 k+4 m-8)},
$$

¿de

1) $b=6$, если $k=m=0$ и $t$ - нескрученный топологический тип;

2) $b=7$ в остальных случаях.

Доказательство аналогично доказательству теоремы 12.1.

Группа $\operatorname{Aut}\left(\Lambda^{2 S}\right)$ действует на $\widetilde{T}^{2 t}$ сопряжениями. Положим

$$
T^{2 t}=\widetilde{T}^{2 t} / \operatorname{Aut}\left(\Lambda^{2 S}\right)
$$

Для любой тройки

$$
\left(z_{1} \mid \theta_{1}^{1}, \theta_{1}^{2}\right),\left(z_{2} \mid \theta_{2}^{1}, \theta_{2}^{2}\right),\left(z_{3} \mid \theta_{3}^{1}, \theta_{3}^{2}\right) \in \mathbb{R}^{(1 \mid 2)}
$$

сушествует $h \in \operatorname{Aut}\left(\Lambda^{2 S}\right)$ такой, что

$$
h\left(z_{1} \mid \theta_{1}^{1}, \theta_{1}^{2}\right)=(\infty \mid 0,0), \quad h\left(z_{2} \mid \theta_{2}^{1}, \theta_{2}^{2}\right)=(0 \mid 0,0), \quad h\left(z_{3} \mid \theta_{3}^{1}, \theta_{3}^{2}\right)=\left(1 \mid \theta^{1}, \theta^{2}\right) .
$$

Таким образом, всякой точке из $T^{2 t}$ отвечает хотя бы одна точка из $\check{T}^{2 t}$. Кроме того, группа

$$
\operatorname{Aut}\left(\Lambda^{2 S}\right)=\left\{h \in \operatorname{Aut}\left(\Lambda^{2 S}\right) \mid h\left(\check{T}^{2 t}\right)=\check{T}^{2 t}\right\}
$$

состоит из отображений, переводящих точки $(\infty \mid 0,0),(0 \mid 0,0),\left(1 \mid \theta^{1}, \theta^{2}\right)$ в точки такого же вида, и, следовательно, порождается отображениями

$$
\begin{aligned}
h_{\lambda}:\left(z \mid \theta^{1}, \theta^{2}\right) & \mapsto\left(z \mid \lambda \theta^{1}, \lambda^{-1} \theta^{2}\right), \\
h^{1}:\left(z \mid \theta^{1}, \theta^{2}\right) & \mapsto\left(z \mid \theta^{2}, \theta^{1}\right), \\
h^{2}:\left(z \mid \theta^{1}, \theta^{2}\right) & \mapsto\left(z \mid-\theta^{1},-\theta^{2}\right) .
\end{aligned}
$$

Группа отображений вида $h_{\lambda}$ эффективно действует на $\check{T}^{2 t}$, если $t$ - тип скрученных пар функций Арфа, и действует тождественно на $\breve{T}^{2 t}$, если $t$ - тип нескрученных функций Арфа.

Согласно теореме 14.1 отсюда следует 
Tеорема 14.2 [43], [52]. Множество $T^{2 t}$ сильно диффеоморфно

$$
\mathbb{R}^{(8 g+4 k+3 m-b(t) \mid 8 g+4 k+4 m-8)} /\left(\mathbb{Z}_{2}\right)^{2},
$$

əде

1) $b(t)=6$, если $k=m=0$ и $t$ - тип нескрученной пары функиий Арфа;

2) $b(t)=8$, если $t$ - тип скрученной парьц функиий Арфа;

3) $b(t)=7$ в остальных случаях.

Таким образом, справедливо

СлеДСТВИЕ 14.1. Пусть $t$ - топологический тип функции Арфа на поверхности типа $(g, k, m)$ и $M^{2 t}$ - множество всех $N=2$ суперримановых поверхностей типа $t$. Тогда $M^{t}$ представляется в виде $T^{2 t} / \mathrm{Mod}^{2 t}$, где $T^{2 t}$ сильно диффеоморфно

$$
\mathbb{R}^{(8 g+4 k+3 m-b(t) \mid 8 g+4 k+4 m-8)} /\left(\mathbb{Z}_{2}\right)^{2},
$$

a группа $\operatorname{Mod}^{2 t}$ дискретно действует на $T^{2 t}$. Eсли $\left(\omega_{1}, \omega_{2}\right)-$ пара функиий Арфа muna $t$, mo

$$
\operatorname{Mod}^{2 t} \cong\left\{\alpha \in \operatorname{Mod}_{g, k, m} \mid \omega_{1} \alpha=\omega_{1}, \omega_{2} \alpha=\omega_{2}\right\}
$$

\section{$\S$ 15. Суперголоморфные морфизмы римановых суперповерхностей}

1. Пусть $\widetilde{U}=\{(\widetilde{z}, \widetilde{\theta})\}$ и $U=\{(z, \theta)\}$ - две суперобласти в $\mathbb{C}^{(1 \mid 1)}$. Рассмотрим отображение $f: U \rightarrow \widetilde{U}$. Оно называется суперконформным, если

$$
\begin{gathered}
\tilde{z}=u(z)-u^{\prime}(z) \varepsilon(z) \theta \\
\tilde{\theta}=\sqrt{u^{\prime}(z)}\left(\theta+\varepsilon(z)+\frac{1}{2} \varepsilon(z) \varepsilon^{\prime}(z) \theta\right),
\end{gathered}
$$

где $u(z) \in L_{0}(\mathbb{C}), \varepsilon(z) \in L_{1}(\mathbb{C})[5]$.

Согласно [6] группа суперконформных автоморфизмов $\Lambda^{s}$ совпадает с $\operatorname{Aut}\left(\Lambda^{s}\right)$.

Пусть теперь $\Gamma \subset \operatorname{Aut}\left(\Lambda^{s}\right)$ - суперфуксова группа, $P=\Lambda^{s} / \Gamma-$ риманова суперповерхность, $\varphi: \Lambda^{s} \rightarrow P$ - естественная проекция и $\varphi^{\#}: \Lambda \rightarrow P^{\#}-$ ее числовая часть. Рассмотрим $U \subset P$, компоненту связности $V$ прообраза $\left(\varphi^{\#}\right)^{-1}\left(U^{\#}\right)$ и $\widetilde{U}=(\#)^{-1}(V)$. Множество $U$ назовем окрестностью точки $p \in U$, если $U=\varphi(\widetilde{U})$. В этом случае отображение $\varepsilon: U \rightarrow \mathbb{C}^{(1 \mid 1)}$ называется локальной картой, если $\varepsilon \varphi: \widetilde{U} \rightarrow \mathbb{C}^{(1 \mid 1)}$ суперконформно и $\varepsilon^{\#}\left(p_{1}^{\#}\right) \neq \varepsilon^{\#}\left(p_{2}^{\#}\right)$ при $p_{1}^{\#} \neq p_{2}^{\#}$. Покрьваюшую $P$ систему локальных карт $\left\{\varepsilon_{i}\right\}$ назовем атласом на $P$.

Пусть теперь $\widetilde{P}$ - другая риманова суперповерхность и $\left\{\widetilde{\varepsilon}_{i}\right\}-$ атлас на ней. Отображение $f: \widetilde{P} \rightarrow P$ назовем суперголоморфнылм морфизмом, если $f(\widetilde{P})=P$ и $f$ суперконформно в картах атласов $\left\{\varepsilon_{i}\right\}$ и $\left\{\widetilde{\varepsilon}_{i}\right\}$. Обратимьй суперголоморфный морфизм назовем суперголоморфным изоморфизмом.

Два суперголоморфных морфизма $f_{1}: \widetilde{P}_{1} \rightarrow P_{1}$ и $f_{2}: \widetilde{P}_{2} \rightarrow P_{2}$ считаются одинаковьми, если сушествуют суперголоморфные изоморфизмы $\widetilde{\omega}: \widetilde{P}_{1} \rightarrow \widetilde{P}_{2}$ и $\omega: P_{1} \rightarrow P_{2}$ такие, что $f_{2} \widetilde{\varphi}=\varphi f_{1}$. Будем говорить, что $f_{1}$ и $f_{2}$ имеют одинаковый топологический тип, если сушествуют гомеоморфизмы $\widetilde{\psi}^{\#}: \widetilde{P}_{1}^{\#} \rightarrow \widetilde{P}_{2}^{\#}$ и $\psi^{\#}: P_{1}^{\#} \rightarrow P_{2}^{\#}$ такие, что $f_{2}^{\#} \widetilde{\psi}^{\#}=\psi^{\#} f_{1}^{\#}$ и $\omega_{1}=\omega_{2} \psi^{\#}$, где $\omega_{i}-$ функция Арфа, отвечаюшая $P_{i}$. 
Суперголоморфный морфизм $f$ назовем супернакрьттием [53], если $f^{\#}$ не имеет критических точек. Всякому суперголоморфному морфизму $f$ отвечает супернакрытие $f_{0}: P \backslash B \rightarrow P^{\prime} \backslash B^{\prime}$, где $B^{\prime}=(\#)^{-1}$ (\{множество критических значений морфизма $\left.\left.f^{\#}\right\}\right)$ и $B=f^{-1}\left(B^{\prime}\right)$. Как и в классическом случае (см. $\left.\S 6\right)$, суперголоморфньй морфизм полностью определяется соответствуюшим супернакрытием и указанием, какие из проколов накрытия $f^{\#}$ надо заклеить. Поэтому в дальнейшем мы рассматриваем только супернакрытия.

ЗАмЕчАНИЕ. В отличие от классического случая в суперслучае не каждый прокол $p \in P^{\#}$ супернакрытия $f: \widetilde{P} \rightarrow P$ можно заклеить. Для этого необходимо и достаточно, чтобы значение функции Арфа $\omega: H_{1}\left(P^{\#}, \mathbb{Z}_{2}\right) \rightarrow \mathbb{Z}_{2}$ на проколе $p$ и значение функций Арфа $\widetilde{\omega}: H_{1}\left(\widetilde{P}^{\#}, \mathbb{Z}_{2}\right) \rightarrow \mathbb{Z}_{2}$ на проколе $\left(f^{\#}\right)^{-1}(p)$ равнялись 0 . При вьполнении первого условия последнее условие эквивалентно нечетности степеней ветвлений в точках $\left(f^{\#}\right)^{-1}(p)$.

2. Перейдем теперь к описанию классов топологической эквивалентности супернакрытий.

Пусть $t$ - топологический тип функции Арфа на поверхности типа $(g, k, m)$. Рассмотрим подгруппу $\gamma \subset \gamma_{g, k+m}=\gamma_{g, n}, \psi \in \widetilde{T}^{t}$ и $P=\Lambda^{s} / \psi\left(\gamma_{g, n}\right)$. Функция Арфа, отвечающая $P$, порождает функцию $\omega: \gamma_{g, n} \rightarrow Z_{2}$. Положим

$$
\widetilde{\operatorname{Mod}}^{(\gamma, \omega)}=\left\{\alpha \in \operatorname{Mod}_{g, k, m} \mid \omega \alpha=\omega, \alpha \gamma=\gamma\right\}
$$

$$
\left.\operatorname{Mod}^{(\gamma, \omega)}=\widetilde{\operatorname{Mod}}^{(\gamma, \omega)} / \widetilde{\operatorname{Mod}}^{(\gamma, \omega)} \cap \operatorname{IMod}_{g, k, m}\right) .
$$

Повторяя рассуждение $\S 6$, находим

Теорема 15.1. Множество супернакрытий одинакового топологического типа образуют связное супермногообразие вида $T^{t} / \operatorname{Mod}^{(\gamma, \omega)}$. Kажсдой паре $(\gamma, \omega)$ отвечает компонента связности пространства суперголоморфных накрытий. Компоненты, отвечающие парам $(\gamma, \omega) u\left(\gamma^{\prime}, \omega^{\prime}\right)$, совпадают, если и только если они переходят друг в друга под действием группь $\operatorname{Mod}_{g, k, m}$.

\section{СПИСОК ЛИТЕРАТУРЫ}

[1] Polykov A. M. Quantum geometry of bosonic string // Phys. Lett. B. 1981. V. 103. P. 207.

[2] Баранов А. М., Шварц А. С. О многопетлевом вкладе в теорию струны // Письма в ЖЭТФ. 1986. Т. 42. № 8. C. 340-342.

[3] Fridan D. Notes on string theory and two dimensional conformal field theory // Proceeding of the Workshop on Unified String Theories. Santa Barbara: Institute for Theoretical Physics, 1985.

[4] Baranov A. M., Manin Yu. I., Frolov I. V., Schwarz A.S. A superanalog of the Selberg trace formula and multiloop contributions for fermionic strings // Comm. Math. Phys. 1987. V. 111. P. 373-392.

[5] Rosly A. A., Schwarz A.S., Voronov A. A. Geometry of superconformal manifolds // Comm. Math. Phys. 1988. V. 119. P. 129-152.

[6] Манин Ю. И. Супералгебраические кривые и квантовые струны // Труды МИАН. 1990. T. 183. C. $126-138$.

[7] Falqui G., Reina C. $N=2$ super Riemann surfaces and algebraic geometry // J. Math. Phys. 1990. V. 31. № 4. P. 948-952. 
[8] Grepner D. Space-time supersymmetry in compactified string theory and superconformal models // Nuclear Phys. 1988. V. 296. P. 757-779.

[9] Hurwitz A. Über Riemannsche Flächen mit gegebenen Verzweigunspunkten // Math. Ann. 1891. V. 39. P. 1-61.

[10] Dubrovin B. Geometry of 2D topological field theories // Integrable Systems and Quantum Groups / ed. M. Francaviglia, S. Greco. Lecture Notes in Math. V. 1620. Berlin: Springer-Verlag, 1996. P. 120-348.

[11] Dubrovin B. Painleve transcendents in two-dimensional topological field theory // Preprint SISSA, 1998. 24/98/FM.

[12] Kontsevich M., Manin Yu. Gromov-Witten classes, quantum cohomology and enumerative geometry // Comm. Math. Phys. 1994. V. 164. №3. P. 525-562.

[13] Ruan Y., Tian G. A mathematical theory of quantum cohomology // J. Differential Geom. 1995. V. 42. № 2. P. 259-367.

[14] Manin Yu., Merkulov S. Semisimple Frobenius (super) manifolds and quantum cohomology of $P^{r} / /$ Topol. Methods Nonlinear Anal. 1997. V. 9. № 1. P. 107-161.

[15] Monastyrsky M. I., Natanzon S. M. The moduli space of superconformal instantons in sigma models // Modern Phys. Lett. A. 1991. V. 6. № 19. P. 1787-1796; № 31. P. 2919.

[16] Monastyrsky M. I., Natanzon S. M. The moduli space of instantons in $N=2$ supersymmetrical $\sigma$-models // Classical Quantum Gravity. 1995. V. 12. № 9. P. 2149-2156; 1996. V. 13. P. 1277.

[17] Monastyrsky M. I., Natanzon S. M. The moduli space of instantons in $N=2$ supersymmetrial $\sigma$-models // Topics in statistical and theoretical physics. F. A. Berezin Memorial Volume. Amer. Math. Soc. Transl. Ser. 2. V. 177. Providence, RI: Amer. Math. Soc., 1996. P. 195-202.

[18] Frike F., Klein F. Vorlesungen über die Theorie der automorphen Funktionen. V. 1, 2. Leipzig: Teubner, 1897, 1912 (Reprinted by Johnson Reprint Corp., New York and Teubner Verlagsges ellschaft. Stuttgart, 1965).

[19] Bers L. Quasiconformal mappings and Teichmüller's theorem // Analytic Functions. Princeton Math. Ser. 1960. V. 24. P. 89-119.

[20] Keen L. Intrinsic moduli on Riemann surfaces // Ann. of Math. 1966. V. 84. № 3. P. 404-420.

[21] Keen L. On Fricke moduli // Ann. of Math. Stud. 1971. V. 66. P. 205-224; A correction to "On Fricke moduli" // Proc. Amer. Math. Soc. 1973. V. 40. P. 60-64.

[22] Wolpert S. The length spectra as moduli for compact Riemann surface // Ann. of Math. 1979. V. 109. P. 323-351.

[23] Натанзон С. М. Пространство модулей вещественных кривых // Труды ММО. 1978. T. 37. C. $219-252$.

[24] Натанзон С. М. Клейновы поверхности // УМН. 1990. Т. 45. №6. С. 47-89.

[25] Натанзон С. М. Пространство модулей вещественных алгебраических $N=1$ и $N=2$ суперкривых // Функц. анализ и его прил. 1996. Т. 30. №4. С. 19-30.

[26] Бобенко А.И. Униформизация Шоттки и конечнозонное интегрирование // Докл. АН CCCP. 1987. T. 295. № 2. C. 268-272.

[27] Натанзон С. М. Примианы вещественных кривых и их приложения к эффективизации операторов Шрёдингера // Функц. анализ и его прил. 1989. Т. 23. № 1. С. 41-55.

[28] Zieschang H., Vogt E., Coldewey H.-D. Surfaces and planar discontionuous groups // Lect. Notes in Math. V. 835. Berlin: Springer-Verlag, 1980.

[29] Натанзон С. М. Инвариантные прямые фуксовых групп // УМН. 1972. Т. 27. № 4. C. $145-164$.

[30] Keen L. Canonical polygons for finitely generated Fuchsian groups // Acta Math. 1966. V. 115. P. 1-16.

[31] Здравковска С. Топологическая классификация полиномиальњых отображений // УМН. 1970. Т. 25. №4. С. 179-180.

[32] Арнольд В.И. Топологическая классификация комплексных тригонометрических многочленов и комбинаторика графов с одинаковым числом вершин и ребер // Функц. анализ и его прил. 1996. Т. 30. № 1. С. 1-17. 
[33] Натанзон С. М. Пространство вещественных мероморфных функций на вещественных алгебраических кривых // Докл. АН СССР. 1984. Т. 279. № 4. С. 803-805.

[34] Натанзон С. М. Топология двумерных накрытий и мероморфные функции на вещественных и комплексных алгебраических кривых. I // Труды семинара по векторному и тензорному анализу с их приложениями к геометрии, механике и физике. Т. 23. М.: МГУ, 1988. C. $79-103$.

[35] Natanzon S. Topology of 2-dimensional coverings and meromorphic functions on real and complex algebraic curves // Selecta Math. Soviet. 1993. V. 12. № 3. P. 251-291.

[36] Натанзон С. М. Униформизация пространств мероморфных функций // Докл. АН CCCP. 1986. T. 287. № 5. C. 1058-1061.

[37] Natanzon S. M. Spaces of meromorphic functions on Riemann surfaces // Topics in Singularity Theory. V.I. Arnold's 60th Anniversary Collection. Amer. Math. Soc. Transl. (2). V. 180. Providence, RI: Amer. Math. Soc., 1997. P. 175-180.

[38] Натанзон С. М. Топологическая структура пространства голоморфных морфизмов римановых поверхностей // УМН. 1998. Т. 53. № 2. С. 155-156.

[39] Dehn M. Über Abbildungen // Mat. Tidsskr. B. 1939. P. 25-48.

[40] Дубровин Б. А., Новиков С. П., Фоменко А. Т. Современная геометрия. М.: Наука, 1982.

[41] Натанзон С. М. Дискретные подгруппы $G L(2, C)$ и спинорные расслоения на римановых и клейновых поверхностях // Функц. анализ и его прил. 1991. Т. 25. № 4. С. 76-78.

[42] Натанзон С. М. Классификация пар функций Арфа на ориентируемых и неориентируемых поверхностях // Функц. анализ и его прил. 1994. Т. 28. № 3. С. 35-46.

[43] Natanzon S. Moduli spaces of Riemann $N=1$ and $N=2$ supersurfaces // J. Geom. Phys. 1993. V. 12. № 1. P. 35-54.

[44] Arf C. Untersuchungen über quadratische formen in Körpern der Charakteristik 2 // J. Reine Angew. Math. 1941. V. 183. P. 148-167.

[45] Натанзон С. М. О квадратичных формах над полем $Z_{2} / /$ УМН. 1995. Т. 50. №5. C. $243-244$

[46] Atiyah M. Riemann surfaces and spin structures // Ann. Sci. Ecole Norm. Sup. 1971. V. 4. P. 47-62.

[47] Johnson D. Spin structures and quadratic forms on surfaces // J. London Math. Soc. (2). 1980. V. 22. P. 365-373.

[48] Mumford D. Theta characteristics of an algebraic curve // Ann. Sci. Ecole Norm. Sup. 1971. V. 4. P. $181-192$.

[49] Натанзон С. М. Пространство Фрике суперфуксовых групп // Функц. анализ и его прил. 1987. T. 21. № 2. C. $80-81$.

[50] Натанзон С. М.Пространство модулей римановых суперповерхностей // Матем. заметки. 1989. Т. 45. № 4. С. 111-116.

[51] Natanzon S. Moduli spaces of Riemann and Klein supersurfaces // Developments in Mathematics: The Moscow School / ed. V. Arnold, M. Monastyrsky. London: Chapman \& Hall, 1993. P. 100-130.

[52] Натанзон С. М. Топологические инварианты и модули гиперболических $N=2$ римановых поверхностей // Матем. сб. 1993. Т. 184. №5. С. 19-38.

[53] Натанзон C. M. Супернакрытия, SNEC-группы и внутренние группы римановых и клейновых поверхностей // УМН. 1990. Т. 45. № 2. С. 217-218.

Независимый московский университет

Поступила в редакцию

E-mail: natanzon@natanzon.mccme.ru

07.12 .1998 